\title{
Error Rate Analysis of NOMA: Principles, Survey and Future Directions
}

This paper was downloaded from TechRxiv (https://www.techrxiv.org).

\section{LICENSE}

CC BY 4.0

SUBMISSION DATE / POSTED DATE

24-01-2022 / 03-02-2022

CITATION

Yahya, Hamad; ahmed, ashfaq; Alsusa, Emad; Al-Dweik, Arafat (2022): Error Rate Analysis of NOMA:

Principles, Survey and Future Directions. TechRxiv. Preprint. https://doi.org/10.36227/techrxiv.18975011.v1

$\mathrm{DOI}$

10.36227/techrxiv.18975011.v1 


\title{
Error Rate Analysis of NOMA: Principles, Survey and Future Directions
}

\author{
Hamad Yahya, Graduate Student Member, IEEE, Ashfaq Ahmed, Senior Member, IEEE, \\ Emad Alsusa, Senior Member, IEEE, and Arafat Al-Dweik, Senior Member, IEEE,
}

\begin{abstract}
Non-orthogonal multiple access (NOMA) has received an enormous attention in the recent literature due to its potential to improve the spectral efficiency of wireless networks. For several years, most of the research efforts on the performance analysis of NOMA were steered towards the ergodic sum rate and outage probability. More recently, error rate analysis of NOMA has attracted massive attention and sparked massive number of researchers whose aim was to evaluate the error rate of the various NOMA configurations and designs. Therefore, the large number of publications that appeared in a short time duration made it highly challenging for the research community to identify the contribution of the different research articles. Consequently, this work aims at surveying the research work that considers NOMA error rate analysis and classifying the contribution of each work. Thus, work redundancy and overlap can be minimized, research gabs can be identified, and future research directions can be outlined. Moreover, this work presents the principles of NOMA error rate analysis.
\end{abstract}

Index Terms-Non-orthogonal multiple access (NOMA), bit error rate (BER), power assignment (PA), enhanced performance.

\section{INTRODUCTION}

$\mathbf{T}$ HE emergence of advanced wireless communications applications has triggered the need to deploy and provide connectivity for a massive number of machine-centric devices, in addition to traditional communications devices such as mobile phones and tablets. Examples of the revolutionary technologies are holographic calls, telemedicine and autonomous systems [1], [2], where Internet of Things (IoT) is considered as the main enabler for most applications [2]. Consequently, the demand for data access has been growing rapidly in the past few years, driven by the large number of new subscribers, high data rate applications, and IoT applications. According to Ericsson mobility report [3], mobile network data traffic grew $44 \%$ between 2020 and 2021, and reached 72 Exabyte per month generated by about 8 billion subscribers. As per Cisco report [4], $50 \%$ of all IoT networked devices in 2023 will be connected to cellular networks. Therefore, the wireless

Hamad Yahya and Emad Alsusa are with the Department of Electrical and Electronic Engineering, The University of Manchester, Manchester M13 9PL, U.K. (email: hamad.mohamadaliyahya@manchester.ac.uk, e.alsusa@manchester.ac.uk)

Ashfaq Ahmed is with the Department of Electrical Engineering and Computer Science, Khalifa University, 127788 Abu Dhabi, UAE (email: ashfaq.ahmed@ku.ac.ae)

Arafat Al-Dweik is with the Center for Cyber Physical Systems (C2PS), Khalifa University, Abu Dhabi 127788, UAE, (e-mail: arafat.dweik@ku.ac.ae), and also with the Department of Electrical and Computer Engineering, Western University, London, ON N6A 3K7, Canada (e-mail: dweik@fulbrightmail.org). network is expected to support about 15 billion IoT devices in addition to 8 billion mobile users. Although the advancements achieved by fifth generation $(5 \mathrm{G})$ wireless networks are considered substantial as compared to the fourth generation (4G), $5 \mathrm{G}$ networks capacity improvement is still way below the $1000 \times$ capacity increase specified by the International Mobile Telecommunications-2020 (IMT-2020) vision.

Non-orthogonal multiple access (NOMA) has attracted tremendous attention as a promising candidate for future mobile networks because of its ability to provide high spectral efficiency, massive connectivity and low latency [5]-[8]. Hence, much research was focused on the integration of NOMA in various applications, such as IoT, short packet communication (SPC), satellite communications, unmanned aerial vehicle communications, visible light communication (VLC), underwater communications, intelligent reflecting surface (IRS) communications and cooperative communications [9]-[13]. Recently, error rate analysis of NOMA has attracted massive attention and motivated massive number of researchers to evaluate the error rate of various NOMA configurations and designs. Therefore, this article summarizes stateof-the-art work related to error rate performance analysis of power and code-domain NOMA.

\section{A. Motivation and Contribution}

In the literature, several survey papers were written to discuss the concepts, advancements, and integration of NOMA into various technologies and applications. All the relevant survey papers of NOMA are listed in Table I, where the focus of each article is emphasized. As can be noted, the error performance of NOMA is not deeply investigated in any survey paper, however, it is briefly discussed in a few cases. For example, the error propagation caused by successive interference cancellation (SIC) and its variation is discussed by Islam et al. [14]. The NOMA resilience to errors caused by imperfect channel state information (CSI) is discussed by Ding et al. [15]. The imperfect SIC propagation errors are discussed by Liu et al. [8]. Furthermore, it is explicitly mentioned that symbol error rate (SER) and bit error rate (BER) are outside the scope of [6].

Based on an extensive literature search, and to the best of the authors' knowledge, there is no survey papers that discuss NOMA error rate performance in a holistic manner. Therefore, this article aims to provide a comprehensive evaluation and classification of the analytical work that considers NOMA error rate performance. More specifically, the main contributions of this work can be summarized as follows: 
TABLE I: Relevant overview papers on NOMA.

\begin{tabular}{|c|c|}
\hline Survey Paper & Area of Focus \\
\hline $\begin{array}{l}\text { Wang et } \quad \text { al. } \\
{[16]-2016}\end{array}$ & $\begin{array}{l}\bullet \text { Compare different NOMA schemes to a gen- } \\
\text { eral NOMA framework } \bullet \text { Summarize NOMA } \\
\text { standardization and prototyping efforts }\end{array}$ \\
\hline $\begin{array}{lrl}\text { Islam et } & \text { al. } \\
{[14]-2017} & \end{array}$ & $\begin{array}{l}- \text { Power-domain based on SC-SIC } \bullet \text { Analysis } \\
\text { of capacity, PA strategies, fairness and user } \\
\text { pairing } \bullet \text { NOMA performance when combined } \\
\text { with other technologies such as cooperative } \\
\text { communication, MIMO, beamforming, space- } \\
\text { time code and network coding }\end{array}$ \\
\hline $\begin{array}{lrr}\text { Ding } & \text { et } & \text { al. } \\
{[15]-2017} & \end{array}$ & $\begin{array}{l}- \text { Power and code-domain } \bullet \text { Design of sin- } \\
\text { gle and multicarrier NOMA } \bullet \text { Integration of } \\
\text { NOMA with MIMO, cooperative communica- } \\
\text { tion and millimeter-wave communication }\end{array}$ \\
\hline $\begin{array}{lrl}\text { Liu } & \text { et } & \text { al. } \\
{[8]-2017} & \end{array}$ & $\begin{array}{l}\text { - Power-domain - Design of NOMA with } \\
\text { multiple antennas, cooperative communication } \\
\text { - Resource management }\end{array}$ \\
\hline $\begin{array}{l}\text { Basharat et al. } \\
{[17]-2018}\end{array}$ & $\begin{array}{l}- \text { NOMA optimization problems including ob- } \\
\text { jective function, constraints, problem type, and } \\
\text { solution approaches } \bullet \text { Performance metrics in- } \\
\text { cluding error rate }\end{array}$ \\
\hline $\begin{array}{lrr}\text { Dai } & \text { et } & \text { al. } \\
\text { [7]-2018 } & \end{array}$ & $\begin{array}{l}\text { Design principles and key features of power } \\
\text { and code-domain } \bullet \text { Summarize performance } \\
\text { in terms of spectral efficiency and receiver } \\
\text { complexity }\end{array}$ \\
\hline $\begin{array}{lcc}\text { Cai } & \text { et } & \text { al. } \\
{[18]-2018} & \end{array}$ & $\begin{array}{l}\text { Major candidates for } 5 \mathrm{G} \text { including power and } \\
\text { code-domain NOMA } \bullet \text { Compare performance } \\
\text { in terms of spectral efficiency, spectral leakage } \\
\text { and BER }\end{array}$ \\
\hline $\begin{array}{lcc}\mathrm{Ye} & \text { et } & \text { al. } \\
{[19]-2018} & \end{array}$ & $\begin{array}{l}- \text { NOMA schemes in different domains } \bullet \text { UL } \\
\text { grant-free NOMA • Operations at BS and } \\
\text { receivers }\end{array}$ \\
\hline $\begin{array}{l}\text { Maraqa et al. } \\
{[6]-2020}\end{array}$ & $\begin{array}{l}\text { Power-domain } \bullet \text { Rate optimization } \\
\text { NOMA combined with other technologies }\end{array}$ \\
\hline $\begin{array}{l}\text { Shahab et al. } \\
{[20]-2020}\end{array}$ & - Design of grant-free NOMA for mMTC \\
\hline $\begin{array}{lll}\text { Makki et } & \text { al. } \\
{[21]-2020} & \end{array}$ & $\begin{array}{l}- \text { Welch-bound equality } \text { spread multiple } \\
\text { access-based NOMA } \bullet \text { BLER performance } \\
\text { and detector complexity }\end{array}$ \\
\hline
\end{tabular}

- Presents the principle operation of NOMA in the downlink (DL) and uplink (UL), and briefly discusses selected NOMA applications.

- Provides a tutorial on BER and SER analysis for NOMA.

- Surveys state-of-the-art work on DL and UL NOMA schemes including optimization oriented work.

- Surveys state-of-the-art work on improved NOMA schemes including labeling and constellation rotation.

- Studies state-of-the-art work on the interplay between NOMA and other technologies including optical, IRS and cooperative communications.

- Identify future research directions and open NOMA research problems.

\section{B. Article Organization}

The rest of the article is organized as follows. Sec. II provides an overview of NOMA principles of operation and considers selected NOMA applications. Sec. III explains the fundamentals of error rate analysis for NOMA. Sec. IV review the state-of-the-art analytical work on the conventional NOMA scheme. Sec. V review. On the contrary, Sec. VI review the improved NOMA schemes. Sec. VII highlights the state- of-the-art analytical work on NOMA combined with other technologies. The future research directions and open research problems are discussed in Sec. VIII. Finally, Sec. IX concludes the article with a summary and the main remarks. Fig. 1 illustrates the core sections of the article.

\section{NOMA OVERVIEW}

This section presents power-domain NOMA main principles of operation at the transmitter and receiver for DL and UL directions.

\section{A. NOMA Principle Operation}

1) Downlink NOMA: Unlike orthogonal multiple access (OMA), NOMA allows the users to share the same resource block in a non-orthogonal manner. For example, the transmitter in DL-NOMA multiplexes the data of $N$ users in the powerdomain by allocating each user a distinct power coefficient, $\alpha_{n}, n \in\{1, \ldots, N\}$. This process is commonly known as superposition coding (SC) and it can be described mathematically described as $x_{s c}=\sum_{n}^{N} \sqrt{\alpha_{n}} x_{n}$, where $\sum_{n} \alpha_{n}=$ 1 to normalize the transmitted power, $x_{n}$ is the $n$th user information symbol, which has a unity average power, i.e. $\mathbb{E}\left[\left|x_{n}\right|^{2}\right], \mathbb{E}(\cdot)$ denotes the statistical expectation process. The information symbols for each user are selected from a symbol alphabet $\chi_{n}$ that corresponds to a certain constellation set such as $M$-ary phase shift keying $\left(M_{n}\right.$-PSK) or $M$-ary quadrature amplitude modulation $\left(M_{n}-\mathrm{QAM}\right)$. The process of allocating the power coefficients and modulation orders is performed by the scheduler at the base station (BS), which is typically configured to ensure fairness and efficient use of resources. Fig. 2a shows the SC process at the BS for the case of $N=2$ where $\alpha_{1}<\alpha_{2}$. The received signal at the $n$th user is $y_{n}=h_{n} x_{s c}+w_{n}$, where $h_{n}$ is the channel coefficient between the BS and $n$th user, and $w_{n} \sim \mathcal{C N}\left(0, N_{0}\right)$ is the additive white Gaussian noise (AWGN). The received signal at the $n$th user is given by

$$
y_{n}=\underbrace{h_{n} \sum_{i=1}^{n-1} \sqrt{\alpha_{i}} x_{i}}_{\text {weak IUI }}+\underbrace{h_{n} \sqrt{\alpha_{n}} x_{n}}_{\text {desired signal }}+\underbrace{h_{n} \sum_{i=n+1}^{N} \sqrt{\alpha_{i}} x_{i}}_{\text {strong IUI }}+w_{n} .
$$

As can be noted from (1), all users experience two types of interference, weak inter-user interference (IUI) and strong IUI, except for the first and $N$ th users whose received signals suffer only from one interference type, and can be expressed as

$$
y_{1}=\underbrace{h_{1} \sqrt{\alpha_{1}} x_{1}}_{\text {desired signal }}+\underbrace{h_{1} \sum_{n=2}^{N} \sqrt{\alpha_{n}} x_{n}}_{\text {strong IUI }}+w_{1}
$$

and

$$
y_{N}=\underbrace{h_{N} \sum_{n=1}^{N-1} \sqrt{\alpha_{n}} x_{n}}_{\text {weak IUI }}+\underbrace{h_{N} \sqrt{\alpha_{N}} x_{N}}_{\text {desired signal }}+w_{N} .
$$

The IUI is classified as strong or weak based on its power as compared to the desired signal power. For the example 


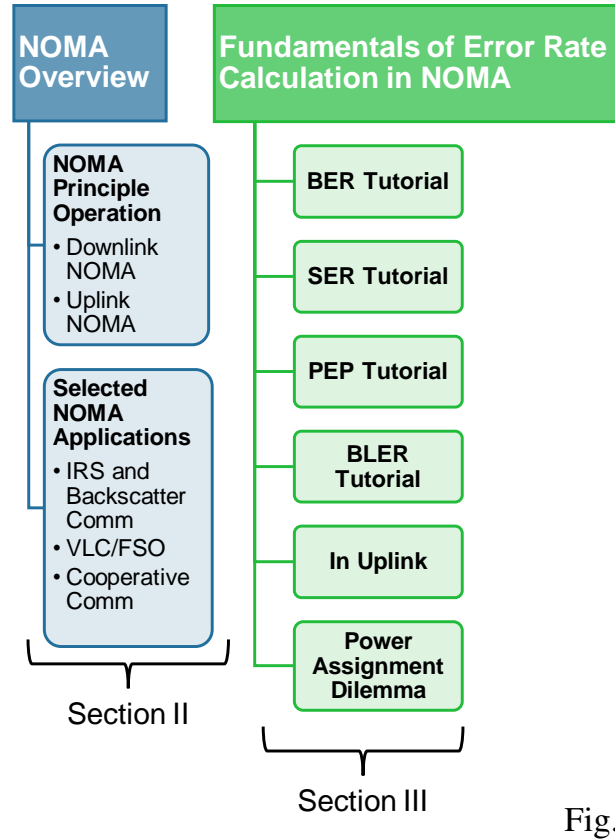

Fig. 1: The structure of the paper.

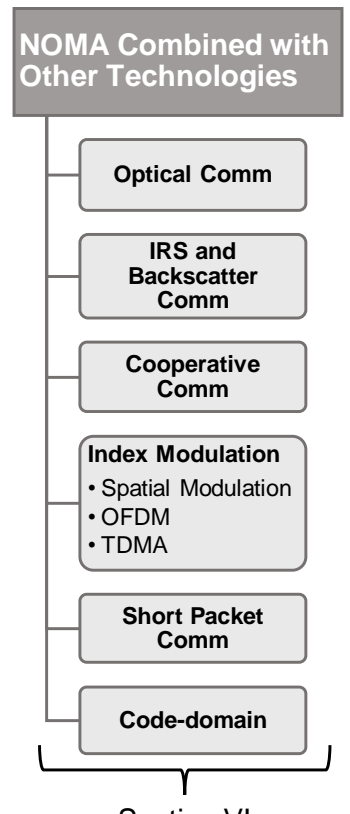

Section VI

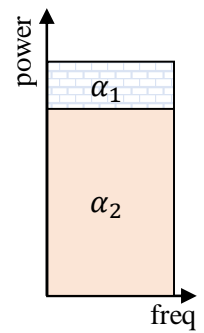

(a)

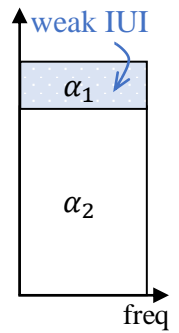

(b)

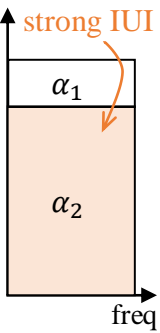

(c)

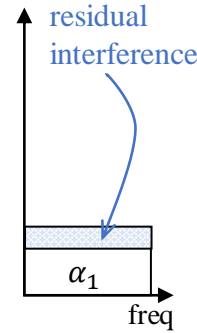

(d)

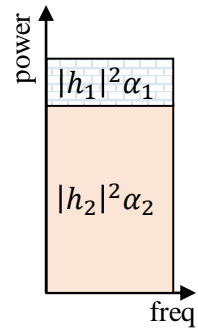

(a)

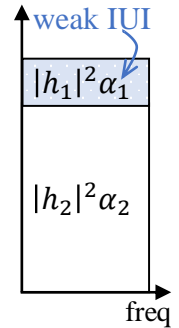

(b)

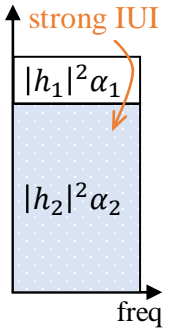

(c)

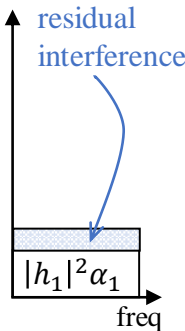

(d)

Fig. 2: Observing DL SC at (a) the BS. (b) user 2. (c) user 1 before SIC. (d) user 1 after SIC.

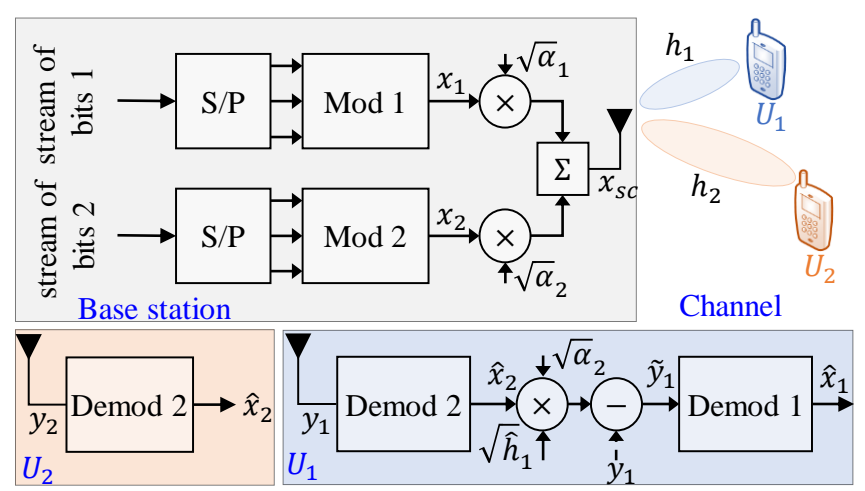

Fig. 3: SC and SIC schematic for $N=2$ case.

shown in Fig. 2, $\alpha_{1}$ is considered as a weak IUI to $x_{2}$ as seen in Fig. 2b. Hence, it is treated as unknown additive noise. Therefore, the user with the maximum power, i.e., user $N$ is interference-limited user, and its symbols can be detected at all users directly using a single-user maximum likelihood detector
Fig. 4: Observing UL SC (a) at the BS. (b) when detecting $x_{2}$. (c) when detecting $x_{1}$ before SIC. (d) when detecting $x_{1}$ after SIC.

(MLD) such that

$$
\hat{x}_{N}=\arg \min _{x_{N} \in \chi_{N}}\left|y_{N}-\hat{h}_{N} \sqrt{\alpha_{N}} x_{N}\right|^{2}
$$

where $\hat{h}_{n}$ is the estimated channel coefficient, which can be written as $\hat{h}_{n}=h_{n}+\check{h}_{n}$ and $\check{h}_{n}$ is the estimation error. This equation can be understood as follows, the most likely $\hat{x}_{2}$ is the trial that results in a minimum Euclidean distance (ED).

On the other hand, $\alpha_{2}$ causes a strong IUI to $x_{1}$ as seen in Fig. 2c. Hence, SIC is used to cancel the strong IUI which might be imperfect and causes residual interference due to channel estimation errors and erroneous detection of the strong IUI signals as seen seen in Fig. 2 d. To detect $x_{1}$, user 1 detects the strong IUI first. Then, it reconstructs it and subtracts it from the original signal, which is typically the SIC process. 
Finally, it applies MLD to detect $x_{1}$. This is described as

$$
\hat{x}_{1}=\arg \min _{x \in \chi_{1}}|y_{1}-\underbrace{\hat{h}_{1} \sqrt{\alpha_{2}} \hat{x}_{2}}_{\text {residual interference }}-\hat{h}_{1} \sqrt{\alpha_{1}} x|^{2}
$$

Fig. 3 shows a schematic of the SC process at the BS for $N=2$ and the detection process at the users as explained in (4)-(5). Alternatively, joint-multiuser maximum likelihood detector (JMLD) can be applied to detect the signals jointly such that

$$
\left\{\hat{x}_{1}, \hat{x}_{2}\right\}=\arg \min _{x_{i} \in \chi_{i}}\left|y_{n}-\hat{h}_{n} \sum_{i=1}^{2} \sqrt{\alpha_{i}} x_{i}\right|^{2}
$$

2) Uplink NOMA: On the other hand, NOMA in the UL uses the channel to multiplex the different users' signals. For instance, the received signal at the BS for the synchronized UL NOMA for $N=2$ case is given as

$$
y=\underbrace{h_{1} \sqrt{\alpha_{1}} x_{1}}_{\text {weak IUI }}+\underbrace{h_{2} \sqrt{\alpha_{2}} x_{2}}_{\text {strong IUI }}+w
$$

Hence, the $n$th user's received signal power at the BS depends on the $\left|h_{n}\right|^{2} \alpha_{n}$. Therefore, the SIC decoding order should depend on the received signal power. Assuming that $\left|h_{2}\right|^{2} \alpha_{2}>\left|h_{1}\right|^{2} \alpha_{1}$. Hence, observing the SC at the BS can be seen in Fig. 4a. When detecting $x_{2}$, the IUI caused by user 1 is weak as seen in Fig. 4b, while the IUI caused by user 2 is strong when it comes to detecting $x_{1}$ as seen in Fig. 4c. Therefore, using SIC to remove the interference might result in residual interference which can be seen in Fig. 4 d. Detecting user's 2 signal can be done as follows,

$$
\hat{x}_{2}=\arg \min _{x \in \chi_{2}}\left|y-\hat{h} \sqrt{\alpha_{2}} x\right|^{2}
$$

while the detection of user's 1 signal can be done using SIC such that

$$
\hat{x}_{1}=\arg \min _{x \in \chi_{1}}|y-\underbrace{\hat{h} \sqrt{\alpha_{2}} \hat{x}_{2}}_{\text {residual interference }}-\hat{h} \sqrt{\alpha_{1}} x|^{2}
$$

Alternatively, JMLD can be used,

$$
\left\{\hat{x}_{1}, \hat{x}_{2}\right\}=\arg \min _{x_{i} \in \chi_{i}}\left|y_{n}-\sum_{i=1}^{2} \hat{h}_{i} \sqrt{\alpha_{i}} x_{i}\right|^{2} .
$$

It is worth mentioning that this section considered $N=2$ case with single antenna setup at the transmitter and receiver ends. For instance, the SIC chain will get longer if more users are considered. Hence, the complexity and delay will increase. The expressions mentioned can be extended easily to multiple users and multi-antennas, and the interested readers are referred to for further details [22], [23].

\section{B. Selected NOMA Applications}

1) IRS and Backscatter Communication: IRS and backscatter communication are recognized to be key enablers to enhancing the spectrum and energy efficiencies for future wireless communication systems [24], [25]. The operation principle of these devices is based on reflecting the incident signals toward an intended destination, where IRS aims to improve the signal-to-noise ratio (SNR) at the destination, while backscatter communication aims to reflect radio frequency (RF) signal to passive or low-power backscatter communication device. The IRS consists of passive metasurface elements that control the reflection angle of the incident signals, whereas the backscatter communication device consists of an antenna and an energy harvesting $(\mathrm{EH})$ circuit with or without batteries to power the communication and sensory systems.

Combining NOMA with IRS or backscatter communication system will improve the coverage and the energy efficiency of the system in general. Furthermore, the IRS-assisted NOMA system with multiple transmitting antenna will allow channel manipulation to achieve the capacity region [26]. Therefore, NOMA combined with IRS or backscatter communication is considered energy, spectrum and cost efficient solution for future wireless networks.

2) VLC and FSO: The evolution of light emitting diodes (LEDs) and photo-detectors have made it possible to deploy light sources for communication [27], [28]. Unlike the optical fiber communication, the VLC and free space optical (FSO) communication takes place wirelessly, where the former is mostly used for indoor mobile users applications, while the latter is used for outdoor applications such as backhauling for fixed point-to-point communication [29]. The frequency band for the VLC is $430 \mathrm{THz}$ to $790 \mathrm{THz}$ which corresponds to the unlicensed visible light spectrum, while the band for FSO includes the infra-red and ultraviolet as well. Such a wide free spectrum can provide a promising solution to the spectrum scarcity problem. Nonetheless, the main limitation of VLC and FSO systems is that the achievable data rates are constrained by the modulation bandwidth of the LEDs which spans to few hundred megahertz. It is worth noting that NOMA is particularly suitable for DL VLC systems that aim to connect few users in a small cell, which is aligned with the principle of NOMA and small cell densification.

3) Cooperative Communication: Cooperative communication is an effective solution to extend the coverage and overcome the channel impairments such as fading, pathloss and shadowing. Two main categories are considered in the literature which are user relaying and dedicated relaying [30]. Users collaborate as relays in the former, while in the latter category, dedicated relay nodes are distributed in the network. Two time slots are needed to relay the signal to the destination when half-duplex (HD) mode is considered. Whereas one time slot is needed by the full-duplex (FD) mode. It is worth noting that higher complexity and self-interference are two limitations for the FD mode. A simple network structure for dedicated user relaying in NOMA is that the near user acts as a HD relay for the far user either through decode-and-forward (DF) or amplify-and-forward (AF). In AF, a scaled version of the received signal is sent to the destination, while an attempt to decode the signal is made then retransmission of the decoded information takes place for DF. Various network structures are discussed in [30]. 


\section{Fundamentals of ERROR RATE CALCUlations IN NOMA}

Various fundamental error rate performance metrics have been considered in the literature to provide insights about the system performance under different conditions and scenarios. These metrics are: BER, SER, pairwise error probability (PEP) and block error rate (BLER). For instance, the BER requires studying the transmitted signal's constellation diagram on the bit-level, whereas the SER computation is done on the symbollevel. The PEP is usually employed as an upper bound of the BER and SER when their computations are too complex because of the constellation diagram shape. Additionally, the BLER is used for coded systems that use block codes assuming fixed channel fading during the block transmission. In the following subsections, a brief tutorial will be given to review the calculation of the previous metrics for NOMA in the downlink direction.

\section{A. BER Tutorial}

To find the BER, then the following steps should be considered:

- Divide the constellation diagram to regions using maximum likelihood (ML) rules. The regions are $\mathcal{R}_{k}^{(1)}$ where $b_{k}=1$ and $\mathcal{R}_{k}^{(0)}$ where $b_{k}=0$. Note that $k \in(1, \ldots, \mathcal{M})$ and $\mathcal{M}=\sum_{n} \mathcal{M}_{n}=\sum_{n} \log _{2} M_{n}$

- Find the probability that for a given transmitted symbol, $b_{k}$ is incorrectly received. In other words, $\operatorname{Pr}\left(b_{k} \neq \hat{b}_{k} \mid x_{s c} \rightarrow b\right)$ where $b$ is the binary representation of the NOMA symbol.

- Due to symmetry, repeat the previous steps for all NOMA symbols in the first quadrant only, and the BER of the specific bit would be the average of all these probabilities because equally likely symbols are assumed.

- The user's BER would be the average of its all bits' BERs.

To illustrate this, let us consider the case shown in Fig. 5a where two NOMA users are using quadrature-phase shift keying (QPSK). The red cross represents the symbols of user 2 which is given a higher power coefficient, while the black squares represent the NOMA symbol. The amplitudes mentioned can be understood using the following example,

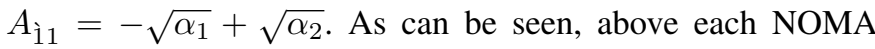
constellation point the binary representation, $b$, is given where the first two bits belong to user 1 while the last belong to user 2. Considering the the first bit of user 2 , which is $b_{3}$. As noted from Fig. 5a, $\mathcal{R}_{3}^{(1)}$ lays in the negative part of the quadrature-axis, whereas $\mathcal{R}_{3}^{(0)}$ is in the positive part. Meaning that for a given channel realization, the imaginary component of the AWGN, $\tilde{w}_{n}$, will decide whether the transmitted bit is received correctly or not. Therefore,

$$
\begin{aligned}
& \operatorname{Pr}\left(b_{3} \neq \hat{b}_{3} \mid x_{s c} \rightarrow 0100\right)= \\
& \quad \operatorname{Pr}\left(b_{3} \neq \hat{b}_{3} \mid x_{s c} \rightarrow 0000\right)=\operatorname{Pr}\left(\tilde{w}_{n}<-A_{11}\right)
\end{aligned}
$$

Similarly,

$$
\begin{aligned}
& \operatorname{Pr}\left(b_{3} \neq \hat{b}_{3} \mid x_{s c} \rightarrow 1100\right)= \\
& \quad \operatorname{Pr}\left(b_{3} \neq \hat{b}_{3} \mid x_{s c} \rightarrow 1000\right)=\operatorname{Pr}\left(\tilde{w}_{n}<-A_{\grave{1} 1}\right)
\end{aligned}
$$

The same steps are repeated for $b_{4}$ where the results are found identical to (11)-(12). Since circularly symmetric AWGN is assumed, the real and imaginary components are random variables with $\mathcal{N}\left(0, N_{0} / 2\right)$. Hence, these probabilities can be calculated as follows

$$
\operatorname{Pr}\left(\tilde{w}_{n}<-\mathcal{A}\right)=\operatorname{Pr}\left(\breve{w}_{n}<-\mathcal{A}\right)=Q\left(\sqrt{\mathcal{A}^{2} \gamma_{n}}\right)
$$

where $\breve{w}_{n}$ is the real component of the AWGN, $\gamma_{n}=\frac{2 \mathbb{E}\left[\left|h_{n}\right|^{2}\right]}{N_{0}}$. Therefore, the BER for user 2 is computed by finding the average of (11)-(12). Hence,

$$
P_{B_{2}}=\frac{1}{2} \sum_{i=1}^{2} Q\left(\sqrt{\mathcal{A}_{i, 2}^{2} \gamma_{2}}\right)
$$

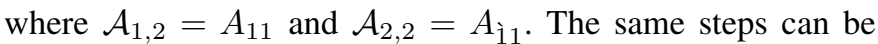
applied for for different labeling schemes and for other users in different $N$ settings when considering JMLD. The readers are referred to references [22], [23], [31] for more details about the derivations when considering SIC and higher modulation orders.

\section{B. SER Tutorial}

The SER of each user can be found by considering the following steps:

- Divide the constellation diagram to regions, $\mathcal{R}_{n}^{\left(s_{n}\right)}$ where $x_{n} \rightarrow s_{n}$ and $s_{n} \in\left(0, \ldots, M_{n}-1\right)$ is the decimal representation of the $n$th user's symbol.

- Find the probability that a given transmitted symbol is correctly received, i.e.

$$
\operatorname{Pr}\left(x_{n}=\hat{x}_{n} \mid x_{s c} \rightarrow s\right)=\operatorname{Pr}\left(y_{n} \in \mathcal{R}_{n}^{\left(s_{n}\right)} \mid x_{s c} \rightarrow s\right)
$$

where $s$ is the decimal representation of the NOMA symbol.

- Find the complementary of the previous probability such that

$$
\operatorname{Pr}\left(x_{n} \neq \hat{x}_{n} \mid x_{s c} \rightarrow s\right)=1-\operatorname{Pr}\left(x_{n}=\hat{x}_{n} \mid x_{s c} \rightarrow s\right)
$$

- Repeat the previous steps for all NOMA symbols in the first quadrant, and the SER would be the average of all these probabilities because equally likely symbols are assumed.

To illustrate this, let us consider the same example used for the BER tutorial. The decimal representation of user $1, s_{1}$, can be found by converting $b_{1} b_{2}$ to decimal, whereas $s_{2}$ can be found by converting $b_{3} b_{4}$ to decimal. Therefore, the regions are found to be: $\mathcal{R}_{2}^{(0)}$ which is the first quadrant, $\mathcal{R}_{2}^{(1)}$ which is the second quadrant, $\mathcal{R}_{2}^{(2)}$ which is the fourth quadrant and 
$\mathcal{R}_{2}^{(3)}$ which is the third quadrant. Furthermore, the probability of correctly received symbol for $x_{s c} \rightarrow 0$ is given as

$$
\begin{aligned}
& \operatorname{Pr}\left(y_{2} \in \mathcal{R}_{2}^{(0)} \mid x_{s c} \rightarrow 0\right) \\
& =\operatorname{Pr}\left(\tilde{w}_{2}>-A_{11} \cap \breve{w}_{2}>-A_{11}\right) \\
& =\operatorname{Pr}\left(\tilde{w}_{2}>-A_{11}\right) \operatorname{Pr}\left(\breve{w}_{2}>-A_{11}\right) \\
& =\left(1-Q\left(\sqrt{\mathcal{A}_{1,2}^{2} \gamma_{2}}\right)\right)^{2}
\end{aligned}
$$

The same can be repeated for all other symbols in the first quadrant. Therefore, the SER of user 2 is found by averaging over the complements of $\operatorname{Pr}\left(y_{2} \in \mathcal{R}_{2}^{(0)} \mid x_{s c} \rightarrow s\right)$, $s \in(0,4,8,12)$. Thus,

$$
\begin{aligned}
P_{S_{2}}=Q( & \left.\sqrt{\mathcal{A}_{1,2}^{2} \gamma_{2}}\right)+Q\left(\sqrt{\mathcal{A}_{2,2}^{2} \gamma_{2}}\right) \\
& +\frac{1}{2} Q\left(\sqrt{\mathcal{A}_{1,2}^{2} \gamma_{2}}\right) Q\left(\sqrt{\mathcal{A}_{2,2}^{2} \gamma_{2}}\right) \\
& -\frac{1}{4} Q^{2}\left(\sqrt{\mathcal{A}_{1,2}^{2} \gamma_{2}}\right)-\frac{1}{4} Q^{2}\left(\sqrt{\mathcal{A}_{2,2}^{2} \gamma_{2}}\right)
\end{aligned}
$$

These steps can be applied for user 1 and for different labeling schemes. For further reading please refer to [32]-[35].

\section{PEP Tutorial}

The union of PEPs is an upper bound of the BER and SER. The PEP considers a pair of symbols such that $x_{n} \neq \check{x}_{n}$ where the decision on the pair is erroneous if $\tilde{w}_{n}>\frac{\Delta_{n}}{2}, \Delta_{n}=$ $\left|x_{s c}-\check{x}_{s c}\right|$ given that $x_{n} \neq \check{x}_{n}$. Therefore, the PEP is simply $Q\left(\frac{\Delta_{n}}{2} \sqrt{\gamma_{n}}\right)$. For further reading please refer to [36]-[39].

\section{BLER Tutorial}

The BLER can be computed by either considering the BER or SER where both lead to the same final solution. Considering the BER then the block is in error if all bits in the block are in error. In other words, $P_{e_{n}}=1-\left(1-P_{B_{n}}\right)^{L \mathcal{M}_{n}}$ where $L$ is the block length in symbols. On the other hand, when considering the SER, the BLER can be computed as $P_{e_{n}}=1-$ $\left(1-P_{S_{n}}\right)^{L}$. Note that this approach can be applied to uncoded systems, whereas some approximations are applied to coded SPCs as will be discussed in the following sections. It is worth noting that all metrics discussed in this section are conditioned for a given $\gamma_{n}$. Hence, to derive the average performance, then an integration over the probability density function (PDF) of $\gamma_{n}$ should take place. For instance, the average BLER can be computed as $\bar{P}_{e_{n}}=\int_{0}^{\infty} P_{e_{n}} \times p_{\gamma_{n}}\left(\gamma_{n}\right) d \gamma_{n}$.

\section{E. In Uplink}

When considering the analysis for given channel realizations, the DL analysis can be extended to UL directly. However, to get the average performance, then $N$-fold integration should be considered because $N$ channel realizations affect the received signal at the BS. This makes the analysis challenging, therefore, different approaches are introduced to solve this issue. For instance, the authors of [40] used union bound for the outer integration and exponential bound for the inner integration. Then applied the total probability theorem and
Tylor series to get the average BER. Kara and Kaya in [31] utilized the fact that the channel realizations are independent and got the PDF of the joint channels. Hence, they were able to get the exact average BER in a single-integral form while an approximate closed-form was also derived.

\section{F. Power Assignment Dilemma}

There are two main power assignment (PA) schemes which are fixed PA and adaptive PA. The former ensures minimum signalling overhead and low complexity owing to its nonoptimality, whereas the latter can ensure optimality or suboptimality but with higher signalling overhead and complexity [22]. Few issues should be considered when designing both schemes. First, ambiguity at the receivers. For example, ambiguity can be caused at the receivers for two NOMA users with binary phase shift keying (BPSK) if equal power coefficients are given when $x_{1}=-x_{2}$. Hence, two NOMA symbols will coincide on the same constellation point.

Second, enabling reliable detection using SIC requires abiding by certain power coefficient bounds (PCBs) [22], [32], [41], [42]. Considering the previous example, when $\alpha_{1}<\alpha_{2}$ the order of the NOMA symbols is as follows from negative inphase axis: $11,01,10,00$. In such scenario, MLD can detect $b_{2}$ reliably because $b_{2}=0$ is always in the positive side. Hence, SIC can cancel the interference caused by $b_{2}$ and detect $b_{1}$ reliably. In contrast, when $\alpha_{1}>\alpha_{2}$, the two middle symbols swap order causing $b_{2}=0$ to fall in two different regions. Therefore, MLD will not be able to detect $b_{2}$ correctly, and hence, SIC performance will be unreliable. It is worth noting that this problem is not faced when JMLD is used.

\section{Conventional NOMA Scheme}

The error rate performance of the conventional NOMA has been widely studied in the literature focusing mainly on the DL with little attention on the UL. Additionally, power allocation and modulation orders optimization based on error performance metrics attracted the attention of many researchers. This section will review the work done for the DL and UL conventional NOMA, as well as its optimization.

\section{A. Downlink}

References considering DL NOMA with various number of users and modulation orders are [22], [31]-[33], [36], [37], [39], [43]-[54].

1) Various Number of Users: The error rate performance of two-user NOMA is extensively in the literature [31]-[33], [43]-[49], unlike the three-user NOMA [36], [37], [50]-[52] and arbitrary number of NOMA users [22], [39], [53], [54]. All the previous references consider single-input-single-output (SISO) channels except [22] which considers arbitrary receiving antennas. Table II provides a comprehensive summary for the work in this part including the error metric under study, antennas setup, channel model, modulation orders, number of users, receiver and CSI availability. It is worthy to mention that this table structure will be adopted throughout the paper. Furthermore, a certain superscript is adopted to distinguish 
TABLE II: Summary of the DL NOMA work.

\begin{tabular}{|c||c||c||c||c||c||c||c|}
\hline$[\#]$ & Metric & Antennas & Channel & $M$ & $N$ & Receiver & CSI \\
\hline \hline$[32]$ & SER & SISO & Rayleigh & $\forall M$ & 2 & Imperf. SIC & Perf. \\
\hline$[33]$ & SER & SISO & AWGN & $\forall M$ & 2 & Imperf. SIC & - \\
\hline$[43]$ & SER & SISO & AWGN & $\forall M \bullet$ & 2 & Imperf. SIC & - \\
\hline$[44]$ & BER & SISO & Rayleigh & $\forall M$ & 2 & Impref. SIC & Perf. \\
\hline$[31]$ & BER & SISO & Rayleigh & $4+2$ & 2 & Imperf. SIC & Perf. \\
\hline$[45]$ & BER & SISO & Rayleigh & $\leq 4$ & 2 & Imperf. SIC & Perf. \\
\hline$[46]$ & BER & SISO & AWGN & 4 & 2 & Imperf. SIC & Perf. \\
\hline$[47]$ & BER & SISO & Rayleigh & 2 & 2 & Perf. SIC & Imperf. \\
\hline$[48],[49]$ & BER & SISO & Rayleigh & 2 & 2 & Perf. SIC & Perf. \\
\hline \hline$[36]$ & PEP & SISO & Nakagami- $m$ & $\forall M$ & 2,3 & Imperf. SIC & Perf. \\
\hline$[37]$ & PEP & SISO & Rayleigh & $\forall M$ & 2,3 & Imperf. SIC & Perf. \\
\hline$[50]$ & PEP & SISO & Rayleigh & $\leq 4$ & 2,3 & Imperf. SIC & Perf. \\
\hline$[51]$ & SER & SISO & Nakagami- $m$ & $\forall M \bullet, \forall M$ & 2,3 & Imperf. SIC & Perf. \\
\hline$[52]$ & BER & SISO & Nakagami- $m$ & 4 & 2,3 & Imperf. SIC & Perf. \\
\hline \hline$[22]$ & BER & SIMO & Rayleigh & $\forall M$ & $\forall N$ & MRC-JMLD & Perf. \\
\hline$[53]$ & BER & SISO & AWGN & 4 & $\forall N$ & Imperf. SIC & - \\
\hline$[54]$ & BER & SISO & Rayleigh & 2 & $\forall N$ & Imperf. SIC & Perf. \\
\hline$[39]$ & PEP & SISO & Nakagami- $m$ & $\forall M$ & $\forall N$ & JMLD, Imperf. SIC, & Perf. \\
\hline
\end{tabular}

TABLE III: Summary of the work considering various channel models for DL SISO NOMA.

\begin{tabular}{|c||c||c||c||c||c||c|}
\hline [\#] & Metric & Channel & $M$ & $N$ & Receiver & CSI \\
\hline \hline$[55]$ & BER & Double Rayleigh & $\forall M^{\bullet}, \forall M^{*}$ & 2 & Perf. SIC & Perf. \\
\hline$[56]$ & BER & $\kappa-\mu$ fading & $4+2$ & 2 & Imperf. SIC & Perf. \\
\hline$[57]$ & BER & $\alpha-\eta-\mu$ fading & 2 & 2 & JMLD & Perf. \\
\hline$[58]$ & BER & $\kappa-\mu$ shadowed fading & $\forall M$ & 2 & Perf. SIC & Perf. \\
\hline$[59]$ & SER & Shadowed Rician & $\forall M^{*}$ & 2 & Perf. SIC & Perf. \\
\hline$[60]$ & BER & $\alpha-\mu$ fading & $\forall M$ & 2,3 & Imperf. SIC & Perf. \\
\hline$[23]$ & BER & Rician & 4 & 2,3 & JMLD & Perf. \\
\hline$[38]$ & PEP & GGN with Rayleigh & 2 & 3 & Imperf. SIC & Perf. \\
\hline$[61]$ & SER & Generalized $K$ & 2 & $\forall N$ & Imperf. SIC & Perf. \\
\hline
\end{tabular}

the modulation schemes, for instance $\star$ is for on-off keying $(\mathrm{OOK}), \bullet$ is for $M$-ary pulse amplitude modulation $\left(M_{n}\right.$ $\mathrm{PAM}), *$ is for $M_{n}$-PSK, $\diamond$ is for $M$-ary pulse position modulation $\left(M_{n}-\mathrm{PPM}\right), \dagger$ is for multiple pulse position modulation (MPPM) and finally no sign is for $M_{n}$-QAM.

Two-user: Starting off with the two-user scenario, the closed-form SER expressions are derived for the imperfect SIC in [32], [33], [43]. The authors of [32], [33] consider rectangular $M_{n}$-QAM, whereas $M_{n}$-PAM is considered in [43]. It is worth noting that the average SER is derived for Rayleigh fading channel in [32], while instantaneous SER is derived in [33], [43].

Turning now to the BER, Assaf et al. [44] derive the exact closed-form BER expressions for imperfect SIC considering rectangular $M_{n}$-QAM. Kara and Kaya [31] derive the BER of the near and far users which use QPSK and BPSK, respectively. Furthermore, the authors of [45] extend the work in [31] to consider the identical BPSK and QPSK cases. The authors of [46] study the high-power amplifier non-linear distortions in a DL orthogonal frequency division multiplexing (OFDM)-NOMA system, where the distortions are modelled with memory polynomial model. The exact closed-form BER expressions are derived for two-user case with QPSK considering perfect and imperfect SIC. Chung [47]-[49] studies the BER performance assuming BPSK for both users and perfect SIC. For instance, [47] quantifies the impact of imperfect CSI, while [48], [49] considers users with correlated information symbols, where [49] studies the negative correlation mapping performance.
Two and three-user: References [36], [52] consider ordered Nakagami- $m$ channels, while references [37], [50], [51] consider Rayleigh fading channel. Bariah et al. [36], [37] derive upper bound BER expressions using the union bound of the PEPs assuming imperfect SIC. Assaf et al. [52] derive exact BER closed-form BER expressions considering QPSK for all users. The authors of [50] propose integrating physical layer security with NOMA to degrade the performance of internal unknown eavesdroppers without affecting the legitimate users performance. The scheme is based on utilizing the random phase of the channel between the users and the base station, which is known to both ends but not to illegitimate users. Hence, the scheme introduces phase shifts of multiples of $2 \pi / M$ to the users' transmitted symbols based on their instantaneous channel phases before SC. Therefore, the eavesdroppers will not be able to decode the symbols as each user's symbol will appear as a different symbol in the constellation diagram. Furthermore, privacy is ensured between the legitimate users. The exact closed-form union bound of the PEPs in insecure conventional NOMA over Rayleigh fading channel is derived for the unknown eavesdroppers considering arbitrary location. Furthermore, worst-case scenario union bound of the PEPs is derived for the proposed scheme.

Reference [51] considers the SER analysis under equally spaced constellations condition because the outer constellation points are only considered in the analysis. Nonetheless, the equal PA for such condition is only found for $M_{n}$-PAM with $M_{1}=M_{2}=2$. Exact and approximate expressions of the SER are presented for two-user case considering $M_{n}$-PAM 
with $M_{n} \in(2,4)$ and $M_{n}$-QAM with $M_{n} \in(4,16)$, while for the three-user case the expressions are presented for $M_{n^{-}}$ PAM with $M_{1}=2, M_{2}=4$ and $M_{3}=8$ only.

Arbitrary number of users: References [22], [53], [54] derive exact closed-form BER expressions of arbitrary number of users, whereas reference [39] considers an upper bound using PEPs. Aldababsa et al. [54] consider users employing BPSK and perfect SIC in Rayleigh fading channels. The authors of [22] consider users with rectangular $M_{n}$-QAM in Rayleigh fading channels. Garnier et al. [53] consider users with QPSK in AWGN channels. The BER is derived considering the real part only as the in-phase and quadraturephase components are independent in QPSK and have identical analysis. Additionally, an iterative algorithm using gradient descent is developed to find optimal power coefficients to minimize the overall system's BER for two and three-users case. The theoretical results are verified by Monte-Carlo simulations and experimental results through software-definedradio testbed.

2) Various Channel Models: Furthermore, various channel models are considered in the literature. For instance, the BER of the two-user case is derived for $\kappa-\mu$ fading [56], $\alpha-\eta-\mu$ fading [57] and $\kappa-\mu$ shadowed fading [58]. In addition, the two-user SER for shadowed Rician fading is derived in [59]. The BER of two and three-user scenarios is considered for $\alpha$ $\mu$ fading channel [60] and Rician fading channel [23]. Bariah et al. [38] derived upper bound BER expressions using the union bound of PEPs considering generalized Gaussian noise (GGN) with Rayleigh fading.

Reference [61] derives approximated and asymptotic SER expressions over generalized $K$ fading channel considering the single user instantaneous SER but with the signal-tointerference-plus-noise ratio (SINR). The PDF of the SINR is derived but it is limited by a boundary condition. While the SER analysis is validated for BPSK with arbitrary number of users, the results are very close from the exact solution. However, this approach is not validated for higher modulation orders. Similarly, the authors of [55] derive the BER of twouser NOMA over double Rayleigh fading channel considering the integration over the SINR PDF. Table III provides a comprehensive summary of the work considering various channel models for DL SISO NOMA.

\section{B. Uplink}

Synchronous UL NOMA is studied considering two-user scenario [62]-[67] and arbitrary number of users [68]. References [62]-[64] derived closed-form BER expressions for SISO AWGN channels, where imperfect SIC is assumed. For instance, both users use QPSK in [62], [63], whereas QPSK and BPSK are assigned to the near and far users to account for channel asymmetry in [64]. Furthermore, accurate BER expressions over fading channels are derived for JMLD in [65], [66], where [65] considers SISO Rician channel and BPSK, while [66] considers single-input-multiple-output (SIMO) Rayleigh fading channel and QPSK.

Liu and Beaulieu [67] derive the closed-form union bounds on the SER and BER of the two-user NOMA with QPSK for arbitrary relative phase offset in AWGN channel. These expressions are valid for both UL and DL, where intentional phase rotation can be applied for the former, and channel can add phase rotation in the UL. Additionally, exact singleintegral-from of the SER and BER expressions are derived for a specific power ratio.

An upper bound of the BER for arbitrary number of UL users with $M_{n}$-PSK in Rayleigh fading channels is derived in [68] considering JMLD and multiple antennas at the base station. It is found that JMLD overcomes the error floor problem of the SIC. Table IV provides a comprehensive summary for the conventional NOMA work in the UL direction.

\section{Optimization}

To improve the performance of NOMA systems, finding the optimal transmission parameters including the power coefficients and modulation orders is considered for different objectives and reliability constraints in [34], [35], [69]-[76]. Table A comprehensive summary for the conventional NOMA optimization work is shown in Table V.

1) Maximum Throughput Design: For instance, references [69]-[71] study resource allocation of two-user DL NOMA system to maximize the sum rate using practical modulation schemes such as $M_{n}$-QAM while satisfying BER constraints. Unlike single carrier systems [71], maximizing the sum rate in multicarrier systems is non-deterministic polynomial-time hard problem which requires highly complex exhaustive search [69], [70]. Therefore, it is important to design efficient algorithms. Cejudo et al. [69] design an efficient resource allocation algorithm to maximize the sum-rate while satisfying the BER constraints. Accurate instantaneous BER expressions are presented for square $M_{n}$-QAM considering imperfect SIC. These expressions are used to derive the exact optimal channel gain ratios between a pair of NOMA users maximizing the achievable sum-rate for a given BER constrain. To ensure reliable SIC detection, the power coefficient for equally-spaced constellation points or highly separable constellation points groups is presented in closed-form. The exact optimal channel gain ratios and the approximated BER expressions over Rayleigh flat fading channel are used to design an efficient iterative resource allocation algorithm which involves user pairing algorithm and continuous power and rate allocation. It is worthy to mention that when NOMA is infeasible, the subcarrier is not used. Moreover, control channel is used to inform the users about the rate, power and subcarrier assignment.

Assaf et al. [70] design an efficient iterative greedy algorithm to maximize the system's throughput while satisfying the users BER constraints. Adaptive modulation, fixed power allocation and hybrid OMA/NOMA are utilized by the design. It is worthy to mention that relaying on exact rather than approximate NOMA BER expressions induce high computational complexity. The authors of [71] propose a joint adaptive power and modulation order to maximize the spectral efficiency without sacrificing the BER performance, where the BER expressions of NOMA over Rayleigh fading are approximated as OMA counterpart. This is applicable only 
TABLE IV: Summary of the UL NOMA work.

\begin{tabular}{|c||c||c||c||c||c||c||c|}
\hline$[\#]$ & Metric & Antennas & Channel & $M$ & $N$ & Receiver & CSI \\
\hline \hline$[62]$ & BER & SISO & AWGN & 4 & 2 & Imperf. SIC & - \\
\hline$[63]$ & BER & SISO & AWGN & 4 & 2 & Imperf. SIC & - \\
\hline$[64]$ & BER & SISO & AWGN & $4+2$ & 2 & Imperf. SIC & - \\
\hline$[65]$ & BER & SISO & Rician & 2 & 2 & JMLD & Perf. \\
\hline$[66]$ & BER & SIMO & Rayleigh & 4 & 2 & JMLD & Perf. \\
\hline$[67]$ & PEP, SER & SISO & AWGN & 4 & 2 & Imperf. SIC & - \\
\hline$[68]$ & PEP & SIMO & Rayleigh & $\forall M^{*}$ & $\forall N$ & JMLD & Perf. \\
\hline
\end{tabular}

TABLE V: Summary of the conventional NOMA optimization work.

\begin{tabular}{|c|c|c|c|c|c|c|c|c|}
\hline$[\#]$ & Objective & Metric & Direction & Channel & $M$ & $N$ & Receiver & CSI \\
\hline [69] & Max. Throughput & BER & $\overline{\mathrm{DL}}$ & Rayleigh & $\overline{\forall M}$ & 2 & Imperf. SIC & Perf. \\
\hline [70] & Max. Throughput & BER & DL & Rayleigh & $\forall M$ & 2 & Imperf. SIC & Perf. \\
\hline [71] & Max. Throughput & BER & DL & Rayleigh & $\forall M$ & 2 & Imperf. SIC & Perf. \\
\hline [72] & Max. Throughput & $\overline{B E R}$ & UL & AWGN, Rayleigh & $\leq 4$ & 2 & Imperf. SIC & Perf. \\
\hline [34] & Min. SER & SER & DL & AWGN & $\forall M^{\bullet}, \forall M$ & 2 & Imperf. SIC & - \\
\hline [35] & Min. BER & SER, BER & $\overline{\mathrm{DL}}$ & AWGN & 4 & 2 & Imperf. SIC & - \\
\hline [73] & Min. BER & SER & DL & AWGN & $\forall M$ & 2 & Imperf. SIC & - \\
\hline [74] & Min. SER & SER & DL & Rayleigh & $\forall M$ & $\forall N$ & Perf. SIC & Imperf. \\
\hline [75] & Min. ED & BER & UL & Rayleigh & $\forall M$ & 2 & JMLD & Perf. \\
\hline [76] & SINR balancing & BER & $\mathrm{DL}$ & Rayleigh & - & 2 & JMLD & Imperf. \\
\hline
\end{tabular}

when assuming perfect SIC. Two adaptive power schemes are proposed to guarantee the minimum target rate for one user while maximizing the rate of the other user. Continuous and discrete adaptive rate modulation are considered to adapt to channel fade level.

On the other hand, adaptive modulation is studied for the UL NOMA in [72], where an asymmetric adaptive modulation algorithm is designed for two-user UL NOMA system to maximize the system throughput for given average SNRs and BER thresholds. The BER expressions are derived in closed-form for AWGN channels considering imperfect SIC and $M_{n}$-PSK with $M_{n}(2,4)$. Lower bound BER expressions are derived for Rayleigh fading channels while ignoring the channel phase effect. The boundary value effect is studied, where to have reliable SIC performance, the modulation orders are selected based on ratio of the users SNRs and the boundary value.

2) Minimum BER Design: References [34], [35], [73], [74] formulate optimization problems to minimize the error performance of DL NOMA systems. For instance, the objective function in [34] is to minimize the instantaneous SER of the strong user while satisfying the SER requirement for the weak user. The instantaneous SER is derived in closedform for both user considering $M_{n}$-QAM and $M_{n}$-PAM. Nonetheless, a closed-form sub-optimal PA is derived. The authors of [35] propose a line search algorithm for a two-user system to find the optimal power coefficients that minimize the un/coded NOMA system's overall error performance in AWGN channels. BER is considered and derived for the uncoded QPSK, while the short packet communication BER approximation is considered for the coded system. The work is extended in [73] to consider square $M_{n}$-QAM, where the BER is approximated using the SER expressions derived for AWGN channels. These expressions are used to find the optimal power coefficients that minimize the instantaneous overall system's BER. The optimization problem is simplified by obtaining upper and lower bounds of the system's overall BER.
Dutta [74] approximates the closed-form BER expressions using the SER expressions that are derived for arbitrary number of DL NOMA users considering arbitrary quadrature amplitude modulation (QAM) and perfect SIC. These expressions have been used for power allocation optimization such that the average BER among all users is minimized while satisfying certain minimum rate requirements per user. Since the problem is non-convex, an upper bound approximation is used for the objective function to make it convex, while the concave rate is transformed to be convex. As the probability of error considers all the possible realizations of the input vector, it is computationally difficult to handle the objective function. Hence, a faster approach is developed to find the optimal power coefficients, which is based on splitting the data sets to subsets and running the optimization for all subsets in parallel.

On the other hand, optimizing the error rate performance of the UL NOMA system is studied in [75] which derives closedform optimal power coefficients and phases to maximize the minimum ED of the sum of the constellations of two-user NOMA with square $M_{n}$-QAM, while JMLD is used at the receiver. The resulting sum constellation is found to be a uniform $M$-QAM constellation of a larger size. Mixed-integer optimization is used to formulate the problem, where the complex Rayleigh fading channel is decomposed to symmetrical real and imaginary parts. Only one part is analyzed due to symmetry.

In a different category, optimal close-form SINR-balancing is derived in [76] for two-user case assuming imperfect CSI. This scheme can provide fairness between the users, however, the performance is not guaranteed.

\section{IMPROVED NOMA SCHEMES}

Improving the error rate performance of NOMA was the main concern of the researchers in the past decade. Thus, several techniques and designs were introduced for NOMA such as labeling, constellation rotation, interference alignment, 


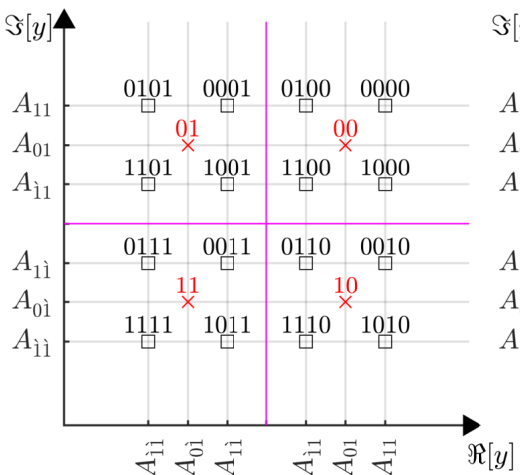

(a)

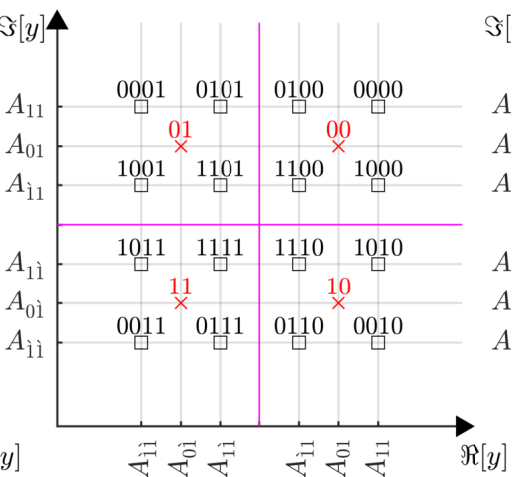

(b)

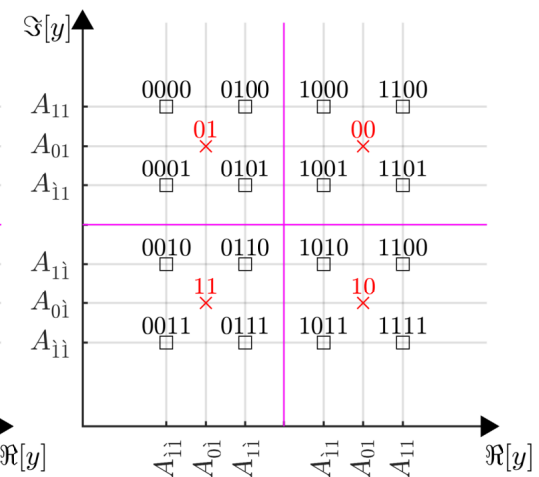

(c)

Fig. 5: NOMA constellation diagram with different symbol-to-bit mapping schemes. (a) NOMA natural labeling. (b) Joint Gray labeling. (c) Natural labeling.

channel coding, multicarrier design, detector design, optical communication, IRS and backscatter communication, cooperative communication, index modulation (IM), short packet communication and code-domain approach. Therefore, in this section we will provide a brief description of each approach and summarize the state-of-the-art research done.

\section{A. Labeling}

The error rate performance of NOMA is highly impacted by the symbol-to-bit labeling strategy used [77]-[80]. There are three main labeling strategies which are: 1) NOMA nonGray labeling, 2) Joint Gray labeling, 3) Natural labeling. The NOMA non-Gray labeling results naturally from the SC process. An example is shown in Fig. 5a. On the other hand, the joint Gray labeling is simply performed such that the NOMA word becomes Gray coded. This is done before SC on each user's stream of bits, $\mathbf{b}_{n}$, such that the stream of bits after joint Gray coding is

$$
\mathbf{g}_{n}= \begin{cases}\mathbf{b}_{n}, & n=N \\ \mathbf{b}_{n} \oplus \mathbf{b}_{n+1}, & 1 \leq n<N\end{cases}
$$

Considering the same example in Fig. 5a, the non-Gray coded NOMA word is 1010 where $\mathbf{b}_{1}=[1,0]$ and $\mathbf{b}_{2}=[1,0]$. Then, $\mathbf{g}_{1}=\mathbf{b}_{1} \oplus \mathbf{b}_{2}=[0,0]$ and $\mathbf{g}_{2}=\mathbf{b}_{2}=[1,0]$. Hence, the jointGray coded NOMA word becomes 0010. Fig. 5b shows the NOMA constellation diagram with joint Gray labeling. As can be seen, a NOMA symbol error results in a single bit error compared to multi bit error for the NOMA non-Gray labeling. Lastly, the natural labeling which is based on natural counting rules and the NOMA constellation for such a labeling scheme is shown in Fig. 5c. While the use of natural labeling imposes the need for JMLD, NOMA with joint Gray labeling does not impose using JMLD. In fact, SIC can still be used, however, decoding should be performed after detection such that

$$
\mathbf{b}_{n}=\left\{\begin{array}{ll}
\mathbf{g}_{n}, & n=N \\
\mathbf{g}_{n} \oplus \mathbf{g}_{n+1}, & 1 \leq n<N
\end{array} .\right.
$$

a) The State-of-the-Art Research on NOMA with Labeling: Reference [77] studies the three labeling schemes for two NOMA users with $M_{n}$-PAM in AWGN channel considering uniform NOMA constellation with a modulation order of $M=M_{1} \times M_{2}$. Meanwhile the simulation results are shown for SER and BLER for different modulation orders, the only closed-form expression derived is the near user SER for $\forall M$ as the far user performance is independent of the labeling scheme. It is observed from the results that compared to other labeling schemes, the joint Gray labeling improves the SER and BLER performance of the near user. Furthermore, the authors of [80] consider Gray-labeling for a DL SIMO system assuming only two users per subcarrier, where the far user is multiplexed with all near users' subcarriers. The BER expressions are derived over Rayleigh fading channels for QPSK and maximal ratio combining (MRC) SIC detector.

Reference [78] derives closed-form expressions of the SER and BER for joint Gray and non-Gray labeling schemes. The derived expressions are for DL two users case with QPSK in AWGN channel. It is noted that error propagation due to SIC imperfections is eliminated at high SNRs when joint Gray labeling is used. This work was extended in [79] by considering arbitrary number of users. The closed-from expressions are presented for specific cases such as the first, second and last users; where the expressions for other users can be inferred. In addition, approximate expressions are demonstrated for the joint Gray labeling. The UL scenario is highlighted for the random phase offset case and proved that the DL expressions can be considered as an upper bound estimation of the UL case for a specific phase offset.

The authors of [81] propose symmetrical coding for twouser DL NOMA with BPSK. The proposed scheme manipulates the transmitted symbol such that the NOMA symbols are Gray-coded for near user as well. Closed-form BER expressions over Nakagami- $m$ fading are derived. Also, an algorithm to optimize the power coefficients based on statistical CSI is developed to achieve better BER performance for the near user. Table VI provides a comprehensive summary for the labeling work.

\section{B. Constellation Rotation}

The principle of constellation rotation in NOMA is to rotate the individual user's constellation before $\mathrm{SC}$ with a specific 
TABLE VI: Summary of the labeling work.

\begin{tabular}{|c||c||c||c||c||c||c||c||c|}
\hline$[\#]$ & Metric & Antennas & Direction & Channel & $M$ & $N$ & Receiver & CSI \\
\hline \hline$[77]$ & SER & SISO & DL & AWGN & $\forall M^{\bullet}$ & 2 & JMLD & - \\
\hline$[78]$ & SER, BER & SISO & DL & Rayleigh & 4 & 2 & Imperf. SIC & Perf. \\
\hline$[79]$ & SER, BER & SISO & DL, UL & AWGN & 4 & $\forall N$ & Imperf. SIC & - \\
\hline$[80]$ & BER & SIMO & DL & Rayleigh & 4 & 2 & MRC-SIC & Perf. \\
\hline$[81]$ & BER & SISO & DL & Nakagami- $m$ & 2 & 2 & Imperf. SIC & Perf. \\
\hline
\end{tabular}
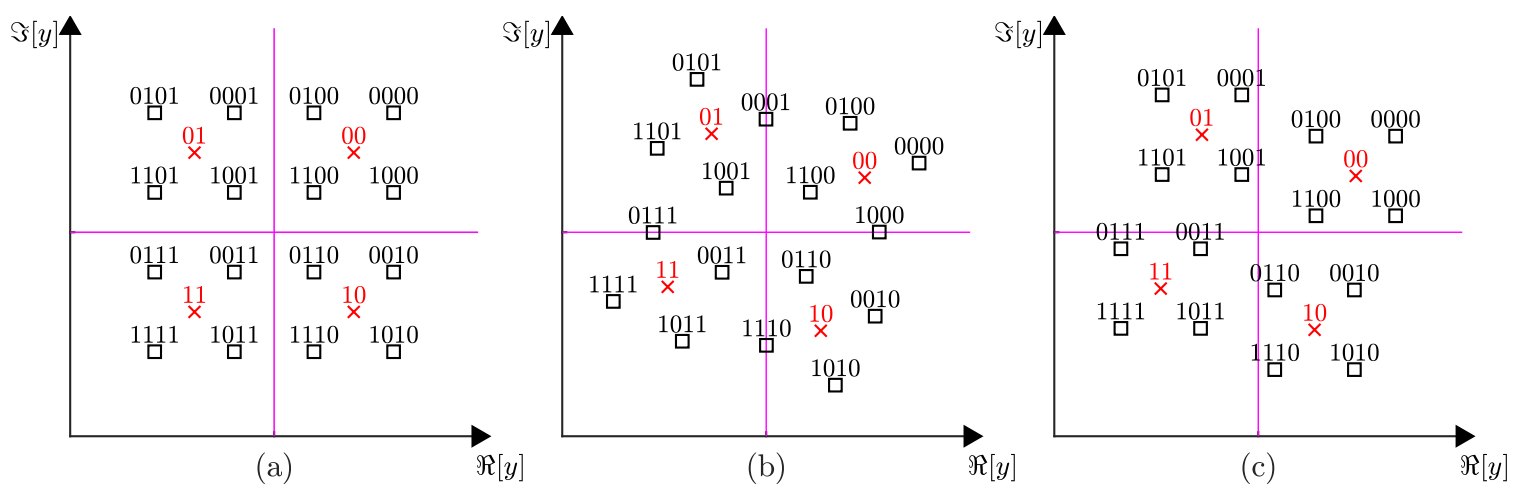

Fig. 6: The constellation diagram of NOMA with constellation rotation utilizing different rotation angles. (a) $\theta_{1}=0^{\circ}, \theta_{2}=0^{\circ}$. (b) $\theta_{1}=30^{\circ}, \theta_{2}=16^{\circ}$. (c) $\theta_{1}=0^{\circ}, \theta_{2}=15^{\circ}$.

angle. For instance, in the DL, the signal transmitted from the $\mathrm{BS}$ is given as

$$
x_{s c}=\sum_{n=1}^{N} \sqrt{\alpha_{n}} x_{n} \exp \left[-\jmath \theta_{n}\right]
$$

where $\theta_{n}$ is rotation angle. This gives extra freedom to optimize the performance. However, extra signalling overhead signalling is introduced besides the higher detection complexity which should be performed jointly. Fig. 6 shows the constellation diagram for the same example as in Fig. 5 where different rotation angles are used. As noted from the figure, the rotation angles do not affect the intra-constellation distances, whereas they directly impact the inter-constellation distances. To understand the meaning of intra-constellation and inter-constellation distances, let us consider Fig. 6a, as can be seen the NOMA constellation can be divided into several group of constellation points that are centered around constellation points of the user with the higher power coefficient. Each group is known as an intra-constellation. For instance, in Fig. 6 there are 4 intra-constellations. In addition, the distance between the intra-constellation points is known as intra-constellation distance. Whereas, the inter-constellation distance is the distance between two constellation points that are from different intra-constellations.

a) The State-of-the-Art Research on NOMA with Constellation Rotation: Reference [82] studies constellation rotation for DL with two users using QPSK. Since deriving exact closed-form expressions of the the SER is challenging due to the arrangement of the NOMA constellation points, upper bound expressions are derived based on the union bound of the PEPs. These expressions are used for rotation angles optimization which is done by exhaustive search. Reference [83] derives the optimal rotation angles that minimizes the ED between the NOMA constellation points for two users case considering identical QPSK and QPSK with 16-QAM. Furthermore, the work is extended to three-user with QPSK in [84], where the optimal phase rotation is found for two schemes which are: 1) rotation for the near user. 2) rotation for the near user and the composite of near and middle users. Reference [85] studies two users NOMA with QPSK and finds the rotation angle that minimizes the dominant PEP, which translate to maximizing the smallest ED. It is found that the optimal rotation angle depends on the ratio of the assigned power coefficients and the phase differences between the channel coefficients for UL, while it only depends on the ration of the assigned power coefficients for DL. The work was extended in [86] by jointly optimizing the power coefficients along-side with the rotation angles. In [87], the authors propose phase pre-distortion for UL VLC-NOMA system to improve the SER. Assuming that the phase difference between the channels of the two users is uniformly distributed, closedform SER expressions are derived considering QPSK for both users and SIC at the receiver. The optimal rotation angle that minimizes the SER is found to be the one that ensures $0^{\circ}$ relative phase, i.e. angle that causes co-phasing. Closedform optimal rotation angles were derived for single and multi-receiving antenna cases. The joint optimization problem reduces to a piece-wise convex optimization problem over its sub-domains, for which the global maximum solution is obtained in closed-form. Reference [88] proposes an algebraic rotation approach to design DL NOMA for two users while achieving full diversity and improved BER performance. This improvement comes from using a special lattice partition scheme that ensures maximum minimum product distance. The upper bound of the minimum product distance is derived in closed-form for arbitrary power coefficients for the two dimensional lattice. The work was generalized in [89] for multi-dimensional lattices. It is worthy to mention that the design and derivations are for JMLD rather than SIC 
TABLE VII: Summary of the constellation rotation work.

\begin{tabular}{|c|c|c|c|c|c|c|c|c|c|}
\hline$[\#]$ & Optimization & Metric & Antennas & Direction & Channel & $M$ & $N$ & Receiver & CSI \\
\hline [82] & PEP & SER, BLER & SISO & $\overline{\mathrm{DL}}$ & Rayleigh & 4 & 2 & $\overline{\mathrm{ML}}$ & Perf. \\
\hline [83] & ED & BER & SISO & $\mathrm{DL}$ & AWGN & 4,16 & 2 & JMLD, Imperf. SIC & - \\
\hline [84] & ED & BER & SISO & $\overline{\mathrm{DL}}$ & AWGN & 4 & 3 & JMLD, Imperf. SIC & - \\
\hline$[85],[86]$ & PEP & BER & SIMO & DL,UL & Rayleigh & 4 & 2 & JMLD & Perf. \\
\hline [87] & SER & SER & SISO & UL & Non-fading & 4 & 2 & Imperf. SIC & Perf. \\
\hline [88] & ED & BER & SISO & UL & Rayleigh & $2-\mathrm{D}$ & 2 & JMLD & Perf. \\
\hline [89] & $\overline{\mathrm{ED}}$ & BER & SISO & $\overline{\mathrm{UL}}$ & Rayleigh & $K-\mathrm{D}$ & 2 & JMLD & Perf. \\
\hline [90] & MI & BER & SIMO & UL & Rayleigh & 4 & 2 & Imperf. SIC & Perf. \\
\hline [40] & - & BER & $2 \times 1$ & $\overline{\mathrm{UL}}$ & Rayleigh & 4 & 2 & JMLD & Perf. \\
\hline [91] & - & SER & SISO & DL & Rayleigh & $\forall M^{\bullet}$ & 2 & MLD & Perf. \\
\hline [92] & SER & SER & SISO & $\mathrm{DL}$ & Nakagami- $m$ & 4 & 2 & MLD & Perf. \\
\hline [93] & ED & SER & SISO & UL & Fading & $\leq 16$ & 2 streams & Imperf. SIC & Perf. \\
\hline [94] & ED & SER & SISO & UL & AWGN & $\leq 4$ & 2 & JMLD & - \\
\hline [95] & ED & BER & SISO & $\mathrm{DL}$ & AWGN & 4 & 2 & JMLD & - \\
\hline
\end{tabular}

which imposes high complexity at the receiver end but with less decoding delay. The results showed that the proposed scheme outperforms the conventional NOMA and the scheme proposed in [82] in fast and block fading channels.

Reference [90] studies two multi-antenna NOMA users in UL with SIC utilizing constellation rotation. Optimal rotation angles are found to maximize the mutual information (MI) where the signal is modeled as Gaussian mixture model. The MI maximization problem is complicated because it involves two random variables. Hence, it is transformed to entropy maximization problem that involves a single random variable only. Efficient closed-form approximation is derived using variational approximation. Improved sum rate and lower BER if noted compared to conventional NOMA. Reference [40] considers constellation rotation with space-time line code (STLC) for two NOMA users in the UL, where the BS is equipped with two receiving antennas and each user is equipped with a single antenna. The channel between each user and each receiving antenna at the BS is assumed to be quasi-static for two time slots and known to the user. Hence, spatial diversity can be obtained using STLC-encoding. Assuming that QPSK is used by both users and constellation rotation is applied on one user only, upper bound expressions of the average BER are derived for JMLD using the union bound of the PEPs. Additionally, an approximation is derived for high SNRs using Taylor series.

The authors of [91] propose a novel scheme for the single antenna DL NOMA based on coordinate interleaving for general number of users, where $M_{n}$-pulse amplitude modulation (PAM) constellation is rotated by $\pi / 4 \mathrm{rad}$ counter clock-wise. Because the real and imaginary components of the $\pi / 4 M_{n}$ PAM constellation are equal, then coordinate interleaving for two users case results in $x_{s c}=\sqrt{\alpha_{1}} \Re\left[x_{1}\right]+\jmath \sqrt{\alpha_{2}} \Re\left[x_{2}\right]+$ $\sqrt{\alpha_{1}} \Im\left[x_{1}\right]+\jmath \sqrt{\alpha_{2}} \Im\left[x_{2}\right]=2 \sqrt{\alpha_{1}} \Re\left[x_{1}\right]+2 \jmath \sqrt{\alpha_{2}} \Re\left[x_{2}\right]$. This results in a $M$-QAM like constellation where $M=M_{1} \times M_{2}$. It is worth noting that $x_{s c}$ in its current form does not have a unity average power. In fact $\mathbb{E}\left[\left|x_{s c}\right|^{2}\right]=2$. Therefore, it has to be normalized for fair comparison with the conventional NOMA system. Additionally, this scheme eliminates the need of using SIC at the receiver for two users case, while it reduces the number of SIC operations by more than half for more than two users as compared to the conventional NOMA system. Furthermore, closed-form expressions of the SER for two users case are derived as well as the optimal power coefficient that achieves fairness.

The authors of [92] generalize the idea in [91] for two users case considering arbitrary rotation angles and more flexible choices of modulation schemes including $M_{n}$-PSK or $M_{n}$ QAM constellation. The need of joint multiuser detection or SIC is eliminated by rotating the constellations of both users and multiplexing the real component of user 1 in the in-phase axis, while the imaginary component of user 2 is multiplexed in the quadrature-phase axis. This results in a $M$-QAM like constellation where $M=M_{1} \times M_{2}$. Nonetheless, the symbolto-bit labeling is unique as user 1 bits flip along the in-phase axis only, while user 2 bits flip along the quadrature-phase axis only. Additionally, the distance between the constellation points is not uniform as it depends on the allocated power coefficients and the rotation angles. The exact instantaneous SER expressions are derived for both users in closed-forms for QPSK case. These expressions are used to find the optimal rotation angles, where the optimal rotation angle is approximated at high SNRs.

Reference [93] studies the UL direction considering a single user having two data streams superimposed using NOMA. The aim is to maximize the minimum inter-constellation distance via constellation rotation, where the rotation angle closed-form expression is derived as a function of the power coefficients for different modulation schemes including BPSK, QPSK and 16-QAM. It is proved that the performance for such design provides more robust error performance and better fairness compared to SNR-dependent designs such as MI maximization. Inspired by [93], the authors of [94] have proposed a novel simple parallelogram-structured constellation design to further improve the SER and maximize the minimum inter-constellation distance. The design is based on shifting one of two coordinates of the constellation allowing arbitrary placement of the constellation points. Furthermore, the work was extended to DL scenario in [95], where the parallelogram-structured constellation is specifically tailored for DL by shifting and scaling the constellation to preserve the parallelogram-structure. The minimum distance is derived in closed-form for the identical QPSK case. Table VI provides a comprehensive summary for the constellation rotation work, where a column for the optimization objective is added. 
TABLE VIII: Summary of the interference alignment work.

\begin{tabular}{|c||c||c||c||c||c||c||c||c||c|}
\hline$[\#]$ & Method & Metric & Antennas & Direction & Channel & $M$ & $N$ & Receiver & CSI \\
\hline \hline$[96]$ & Constellation Rotation & SER & SISO & DL & AWGN & 4 & 2 WIT + 1 WPT & Imperf. SIC & - \\
\hline$[97]$ & Constellation Rotation & SER & SISO & DL & AWGN & $\forall M$ & $N$ WIT + 1 WPT & Imperf. SIC & - \\
\hline$[98]$ & Constellation Rotation & SER & SISO & DL & Rayleigh & 4 & 2 & JMLD & Perf. \\
\hline$[99]$ & Symbol Scaling & BER & SISO & DL & Rayleigh & $\leq 4$ & 2,3 & JMLD & Perf. \\
\hline$[100]$ & Symbol Scaling & BER & SISO & DL & Rayleigh & $2+4$ & 2 & JMLD & Perf. \\
\hline
\end{tabular}

\section{Interference Alignment}

Not all interference cause degradation in the performance of NOMA. In fact, constructive interference improves the performance as the desired signal power is boosted by the interference. On the other hand, destructive interference degrades the performance of NOMA. Therefore, the concept of interference alignment is to align the individual users' signals so they add up constructively. This can be achieved via phase rotation [96]-[98] or symbol scaling [99], [100]. A summary of the work in this part is included in Table VIII.

a) The State-of-the-Art Research on NOMA with Interference Alignment: Reference [96] proposes constellation rotation scheme for simultaneous wireless information and power transfer (SWIPT)-assisted NOMA to constructively maximize the amount of energy carried to the wireless information transfer (WIT) users and wireless power transfer (WPT) users, while satisfying acceptable SER performance for the WIT users. In order to minimize the signalling overhead associated with informing the WIT receivers about the optimal rotation angles, the constellation rotation is performed jointly over a block of symbols rather than on a symbol-by-symbol basis. The optimal constellation rotation angles are derived for QPSK case assuming two NOMA WIT users and a single WPT user. The work is extended in [97] by considering a multi-carrier system, arbitrary modulation orders, and multi WIT and WPT users. A joint design of the energy interleaver and constellation rotation is proposed, where the energy interleaver is introduced to suppress the destructive interference of multiple symbols scheduled on each sub-carrier. Furthermore, the receiver operations for WIT and WPT users as well as the signalling overhead are discussed thoroughly. An iterative algorithm is proposed for obtaining optimal the constellation rotation angles since the objective function is non-convex. Whereas a low-complexity greedy algorithm is proposed for the energy interleaver integer optimization. In [98], phase rotation is applied to the near user to allow constructive interference with the far user's symbol. In addition, a nonredundunt precoding is applied to the near user's symbol to allow symbol extraction at the receiver end. The authors propose a semi-blind detection scheme based on independent component analysis to detect the near user's symbols after rotation. Nonetheless, it requires $1-2$ pilot signals to remove ambiguities caused by the independent component analysis. The SER is derived in closed-form under Rayleigh fading for two users with QPSK assuming perfect ambiguity elimination. Additionally, an efficient power allocation algorithm that is based on statistical channel information is proposed to minimize the average asymptotic SER. Furthermore, the computational complexity of the scheme is studied and compared with other common benchmarks such as [83],
[86].

References [99], [100] propose a data-aware power assignment for DL NOMA to scale the NOMA symbols with different power coefficients in order to maximize the constructive interference. This scheme is based on the polarities of the information symbols real and imaginary components. Hence, the assignment for the in-phase component can be different from the quadrature-phase component. Reference [99] derives the BER expressions for two and three users considering identical BPSK case. In addition, the optimal power coefficients are found. Whereas, Reference [100] derives the BER for two users case considering BPSK for the near user and QPSK for the far user, where the power coefficients that maximize the constructive interference are found.

\section{Channel Coding}

Channel coding is a well known approach to improve the reliability of the communication system with the cost of lower spectral efficiency and higher complexity at the transmitter and the receiver. Thus, studying the complexity and error rate performance of NOMA-based channel coding transceivers is important. Examples of channel codes are low-density paritycheck (LDPC) [101], [104], [105], Reed-Solomon codes [101], trellis codes [102] and polar codes [103]. A comprehensive summary for the NOMA work with channel coding is included in Table IX.

a) The State-of-the-Art Research on NOMA with Channel Coding: The authors of [101] propose a novel linear receiver that exploits the constraints of the forward error correcting (FEC) codes to overcome the SIC error propagation problem caused by the imperfect CSI at the receiver, where the channel is estimated by interpolating the pilot subcarriers estimations. The novel receiver is applicable to symbol and codewordlevel SIC. It is designed for a multiple-input multiple-output (MIMO) two-user UL NOMA based on the minimum output energy criterion that can handle moderate channel estimation errors, where the IUIs are separated from the desired signals by adopting a unique signature based on the FEC codes' constraints. The receiver optimization is formulated into a quadratic programming problem anchored with set of code constraints. Transmit diversity and capacity achieving LDPC codes are considered. To improve the performance further, an iterative receiver is considered to recover the recover the data from the Reed-Solomon code that is serially concatenated to an LDPC code. To verify the reliability of the proposed detector, an upper bound coded BER is derived based on the union bound of the PEPs.

The authors of [102] propose a trellis code modulation for two-user DL NOMA, where the symbols of each user 
TABLE IX: Summary of the channel coding work.

\begin{tabular}{|c||c||c||c||c||c||c||c||c||c|}
\hline$[\#]$ & Coding & Metric & Antennas & Direction & Channel & $M$ & $N$ & Receiver & CSI \\
\hline \hline$[101]$ & LDPC & PEP & MIMO & UL & Rayleigh & 4 & 2 & Novel JMLD & Imperf. \\
\hline$[102]$ & Trellis & BER & SISO & DL & Fading & $\forall M^{*}$ & 2 & Joint Viterbi & Perf. \\
\hline$[103]$ & Polar & BLER & SISO & UL & AWGN & 2 & 2 & Adaptive List SIC & Perf. \\
\hline$[104]$ & Turbo & BLER & SISO & DL & Rayleigh & 4 & 2 & LLR-based & Perf. \\
\hline$[105]$ & LDPC & PEP & SISO & UL & Rayleigh & $\forall M$ & 2 & Perf. Codeword-level SIC & Perf. \\
\hline
\end{tabular}

are modulated independently and multiplexed in the power domain. The detection is performed jointly using the Viterby algorithm where the trellis-coded NOMA is treated as a regular trellis code modulation with the tensor product trellis. Selecting appropriate power coefficients can ensure good decoding performance. Since larger free distance leads to a better performance at high SNR, the optimal power coefficients are found to maximize the free distance of the tensor product trellis. Closed-form expression of the free distance of the tensor product trellis is derived.

The authors of [103] propose polar code for the two-user binary Gaussian multiple access channel, where for each user two blocks of polar codes are constructed with unequal power. Code construction and power and rate allocation are jointly optimized to improve the performance. To maintain fairness, alternating power allocation over the two blocks is proposed. At the receiver side, hard-decision SIC employing successive cancellation based on adaptive list size is proposed. Upper bound BLER expressions are derived for the finite block length regime assuming BPSK.

The authors of [104] design a SIC-free detector for twouser DL NOMA utilizing turbo codes. The proposed detector is based on the log-likelihood ratios (LLRs) for each bit of the NOMA symbol. These LLRs are derived in closed form considering QPSK for both users. The symbol-level and codeword-level SIC detectors are considered as benchmarks.

The authors of [105] propose probabilistic shaping combined with QAM for $N$ UL NOMA users in Rayleigh fading channels to improve the spectral efficiency. Probabilistic shaping assigns the constellation points that have equidistance space different probabilities such that the source generates information symbols with non-uniform probabilities using constant composition distribution matcher method. To maximize the ergodic constrained capacity, a multi-step optimization is performed to find the optimal probability mass function (PMF) for each user where the optimization order is the inverse of the SIC order. At the receiver, maximum a posterior (MAP)-based codeword-level SIC is implemented with an order determined by the large-scale pathloss. Unlike the symbol-level SIC, the codeword-level SIC can ideally cancel the IUI caused by the previously decoded signals due to the powerful error correcting codes as well as the soft channel decoder. Whereas the rest IUIs are assumed to be Gaussian distributed. To validate the reliability of the proposed scheme, closed-form average PEP expressions are derived. Furthermore, a design example combines the proposed system combined with the practical LDPC codes to study the spectral efficiency and evaluate the BER performance using Monte-Carlo simulations.

\section{E. Multicarrier NOMA Design}

In [106], wavelet transform pulse shaping is analyzed for the DL OFDM NOMA as it is more spectrally efficient than the fast Fourier transform (FFT) pulse shaping which requires cyclic prefix. Closed-form BER expressions are derived for both pulse shaping schemes in AWGN channels based on the ED approach, where the presented analysis holds for two-user case with QPSK assuming ideal synchronization. The strong channel user apply SIC to detect its signal, whereas the weak channel user detects its signal assuming the other user's signal being in the noise floor. The effect of the AWGN on the received signal is studied after passing the AWGN components through the discrete Fourier transform (DFT) and discrete wavelet transform (DWT) filter banks and getting their true variances. Since the high frequency components are discarded in the DWT filter banks, the channel effect on the received signal is reduced and the signal recovery is improved.

Maatouk et al. [107] propose combining orthogonal frequency division multiple access (OFDMA) and code division multiple access (CDMA) for DL NOMA. Unlike conventional NOMA which for instance combines two users and uses SIC to cancel the interference, the proposed scheme combines two groups of users with two sets of orthogonal waveforms and uses iterative SIC to cancel the interference between the groups. The users in the OFDMA group can detect their signal directly as the number of the users in the CDMA group is assumed to be small such that the interference is negligible. On the other hand, the users of the CDMA group need to use iterative SIC to cancel the interference. Al-Khansa et al. [108] extened the work by introducing a power split between the two groups that follows the conventional NOMA. Approximate BER expressions are derived for QPSK case in AWGN channel by assuming the interference being a Gaussian-like signal for the OFDM group of users, while the number of possible sequence errors for the CDMA group is assumed to follow the binomial distribution.

The authors of [109] study combining the generalized frequency division multiple access (GFDMA) waveforms with NOMA, where the information symbol in each subcarrier is shaped using a filter. Analytical conditional BER expressions for the DL and UL are derived considering two-users and QPSK for both users. Perfect SIC and imperfect SIC are considered and their BERs are derived. A comprehensive summary for the mutlicarrier NOMA system designs is included in Table X.

\section{F. Detector Design}

This subsection focuses on the innovations in the research to improve the performance of the NOMA detectors in dif- 
TABLE X: Summary of the multicarrier NOMA design work.

\begin{tabular}{|c||c||c||c||c||c||c||c||c||c|}
\hline$[\#]$ & System & Metric & Antennas & Direction & Channel & $M$ & $N$ & Receiver & CSI \\
\hline \hline$[106]$ & Wavelet Transform OFDM & BER & SISO & DL & AWGN & 4 & 2 & Imperf. SIC & Perf. \\
\hline$[107]$ & OFDMA-CDMA & BER & SISO & DL & AWGN & $16+4$ & 2 & Iterative Hard and Soft SIC & Perf. \\
\hline$[108]$ & OFDMA-CDMA & BER & SISO & DL & Rayleigh & 4 & 2 & Iterative Hard and Soft SIC & Perf. \\
\hline$[109]$ & GFDMA & BER & SISO & DL,UL & Rayleigh & 4 & 2 & Imperf. SIC \\
\hline
\end{tabular}

TABLE XI: Summary of the detector design work.

\begin{tabular}{|c||c||c||c||c||c||c||c||c||c|}
\hline$[\#]$ & Carrier & Metric & Antennas & Direction & Channel & $M$ & $N$ & Receiver & CSI \\
\hline \hline$[110]$ & Multi & BER & SISO & UL & Rayleigh & $\forall M$ & 2 & Triangular SIC & Perf. \\
\hline$[111]$ & Multi & BER & SISO & UL & Rayleigh & $\forall M$ & 3 & Triangular SIC & Perf. \\
\hline$[112]$ & Single & BER & SISO & UL & AWGN & 4 & 2 & Time asynchronous SIC & Perf. \\
\hline$[113]$ & Multi & SER & SISO & UL & AWGN & 4 & 2 & JMLD-SIC & \\
\hline$[114]$ & Single & SER & SIMO & DL & Rayleigh & $\forall M$ & 2 & Threshold based & Perf. \\
\hline$[115]$ & Single & BER & SISO & DL & Rayleigh & $\forall M \bullet$ & $\forall N$ & Improved SIC & Perf. \\
\hline
\end{tabular}

ferent scenarios including asynchronous UL communication [110]-[112], synchronous UL communication [113] and DL communication [114], [115]. A comprehensive summary for the NOMA detector designs is included in Table XI.

a) The State-of-the-Art Research on NOMA Detector Design: Haci et al. [110] study the asynchronous UL NOMA system as perfect synchronization is difficult to achieve for UL users distributed in geographically diverse and dynamic environments. They propose a novel SIC detector based on triangular pattern, where iterative soft and hard decision detectors are studied for higher modulation orders. The BER performance analysis is presented in [111] for the three-user case in flat Rayleigh fading considering conventional SIC and the proposed SIC, where besides the receiver power ratios, the BER of the proposed SIC depends also on the iteration number and the time offset between users. It is assumed that the relative time offset between the users is uniformly distributed, and the residual interference is approximated to the Gaussian distribution.

Liu and Beaulieu [112] propose a novel time asynchronous SIC for the asynchronous UL NOMA. The detector is based on the triangular-SIC introduced by [110], [111] where an optimal time-offset is selected to eliminate the IUI. The optimal time offset is found to be half the symbol duration for the QPSK case. It is worthy to mention that the proposed detector does not introduce signalling overhead nor higher complexity, however, it requires the received symbol timing. Exact BER expressions are derived over AWGN channel considering twouser case with QPSK and imperfect SIC, where no relative phase rotation is assumed between the users. Additionally, a simpler BER approximation is derived for the weak user by relaxing the correlation between the noise samples at the detector.

To mitigate the error propagation problem caused by SIC, [113] propose an improved detector to allow much smaller range of power differences between the UL NOMA users, as compared with standard SIC. The proposed detector is based on hard decision JMLD to find decisions on the weak user signal, then using them to cancel the interference from the strong user. Hence, computing the LLRs to find the strong user signal, which is then used to cancel the interference from the weak user signal. Assuming co-phased signals transmitted from the users, the uncoded BER of the standard SIC and the proposed detector are derived considering QPSK for both users.

The authors of [114] propose a low-complexity threshold based detector to detect the signals of two NOMA users in the DL, where the BS is equipped with a single antenna while the users are equipped with multiple antennas and use $M_{n}$-QAM with $M_{n} \in(4,16)$. The exponential bound of the $Q(\cdot)$ function is used to derive the SER expressions for Rayleigh fading channels. Al-Dweik et al. [115] study phaseindependent NOMA using unipolar amplitude shift keying (ASK) which allows amplitude-coherent detection. Considering two and multiuser systems in ordered SISO Rayleigh fading channels, closed-form BER expressions are derived for three types of detectors, namely JMLD, conventional SIC and improved SIC. The improved SIC cancels interference more effectively that conventional SIC and has identical performance to the JMLD but with lower complexity.

\section{NOMA COMBINED WITH OTHER TECHNOLOGIES}

\section{A. Optical Communication}

VLC and FSO assisted NOMA systems fall under the umbrella of optical-NOMA (O-NOMA). A light source like an LED is used to transmit data while photo-detectors are used at the receiver to convert the optical signal to electrical. VLC and FSO are considered promising technologies to improve the spectral efficiency. Additionally, the transmission is highly secure as the light cannot penetrate through the walls, unlike RF signals. Thus, it is foreseen that combining VLC and FSO with NOMA will provide tremendous spectral efficiency improvements to the communication system. A comprehensive summary for the O-NOMA work is included in Table XII.

a) The State-of-the-Art Research on O-NOMA: References [12], [13], [116]-[127] study the error rate performance of the DL VLC-NOMA system. Huang et al. [116] attempt to characterize the SER by analytically deriving the SER expressions considering multiple LEDs, unipolar transmission and line-of-sight (LoS) optical channels that are corrupted by AWGN. The PDF of the optical channel is obtained using change of variable method, while the SER derivation is simplified using convolution theory and subsection integral method. Marshoud et al. [117] derive closed-form BER expressions 
TABLE XII: Summary of the VLC work.

\begin{tabular}{|c||c||c|c||c||c||c||c|c|}
\hline$[\#]$ & Metric & Antennas & Direction & Application & $M$ & $N$ & Receiver & CSI \\
\hline \hline$[116]$ & SER & MISO & DL & Indoor & $2^{\star}$ & $\forall N$ & Imperf. SIC & Perf. \\
\hline$[117]$ & BER & SISO & DL & Indoor & $2^{\star}$ & $\forall N$ & Imperf. SIC & Perf. \\
\hline$[12]$ & BER & SISO & DL & Indoor & $2^{\star}$ & $\forall N$ & Imperf. SIC & Imperf. \\
\hline$[118]$ & BER & SISO & DL & Indoor & $\leq 16^{*}$ & 2 & Imperf. SIC & Perf. \\
\hline$[119]$ & BER & SISO & DL & Indoor & $\leq 16^{*}, \forall M, \forall M M^{\bullet}$ & 2 & Imperf. SIC & Perf. \\
\hline$[120]$ & SER & SISO & DL & Indoor & $\forall M$ & 2 & Imperf. SIC & Perf. \\
\hline$[13]$ & BER & SISO & DL & Underwater & $2^{\star}$ & 2 & Imperf. SIC & Perf. \\
\hline$[121]$ & BER & SISO & DL & Indoor & $2^{\star}$ & $\forall N$ & Sum and Sample SIC & Perf. \\
\hline$[122]$ & SER & SISO & DL & Indoor & $2^{\star}+\forall M^{\dagger}$ & 2 & ML, MLD & Perf. \\
\hline$[123]$ & BER & SISO & DL & Indoor & 4 & 2 & Imperf. SIC & Perf. \\
\hline$[124]$ & BER & $2 \times 2$ & DL & Indoor & $2^{\star}$ & 3 & MRC and SIC & Perf. \\
\hline$[125]$ & BER & SISO & DL & Indoor & $2^{\star}+\forall M^{\diamond}$ & 2 & Imperf. SIC & Imperf. \\
\hline$[126]$ & BER & $2 \times 2$ & DL & Indoor & $2^{\star}+\forall M^{\diamond}$ & 2 & MRC and SIC & Imperf. \\
\hline$[127]$ & BER & MIMO & DL & Indoor & $\forall M$ & 2 & Imperf. SIC & Imperf. \\
\hline$[128]$ & BER & SISO & UL & Space & 2 & 2 & Imperf. SIC & Perf. \\
\hline
\end{tabular}

taking into account perfect and imperfect SIC. The work considers a single LED with arbitrary number of users using unipolar OOK. Marshoud et al. [12] extend the work in [117] to consider noisy CSI as well as outdated CSI, where an approximation is derived for the former while an upper bound is derived for the latter. Additionally, BER expressions are derived for analog and digital dimming control.

The authors of [118] derive closed-form BER expressions for two-user system considering imperfect SIC and a fixed set of $M_{n}$-PSK. The work is extended in [119] to consider modulation schemes such as QAM and PAM. Almohimmah and Alresheedi [120] derive exact closed-form SER for twouser scenario considering imperfect SIC and arbitrary square $M_{n}$-QAM. Similarly, Jain et al. [13] derive closed-form BER expressions considering OOK in underwater environments, where the channel is modeled as exponential generalized gamma distribution. The system is analyzed and validated for parameters such as air bubble levels, gradient temperature and salinity level of the water.

The authors of [121] categorize the users in a cell based on their data rate requirements. Hence, they propose an adjustable bit-rate SC meaning that each user's bit interval is multiple base intervals, where the base interval is the bit interval of the slowest bit rate user. Unlike the conventional SIC, the sampleand-sum SIC is introduced to minimize the error propagation caused by the conventional SIC, where each user samples the SC signal at a certain sample rate. Then, these samples will be summed for decoding. Moreover, a modified fixed power allocation is proposed to provide some clear correlation between the effects of adjusting power coefficients on the BER performance. The authors derive closed-form BER expressions for the two and multiuser with OOK. Furthermore, the authors of [122] propose using OOK for the low data rate user, while MPPM is assigned for the high data rate user. To overcome the error propagation, SIC-free detector is proposed, where the MPPM user uses soft decision detector, whereas the OOK user sums the detected samples and uses MLD to detect its signal. Closed-form SER expression is derived for the OOK user while a single-integral form SER expression is derived for the MPPM user. Furthermore, asymptotic approximation for the probability of the number of wrongly decoded slots in one MPPM symbol is derived.
For a two-user system, the authors of [123] propose adjusting the constellation of one user to improve the overall BER. Closed-form BER expressions are derived considering QPSK for both users, and the convexity the objective function is proved as the Hessian matrix of the objective function is positive semi-definite. Furthermore, the Lagrangian is used to find the optimal constellation parameters, where the optimal parameters are presented for various SNRs.

Dixit et al. [124] analyze repetition code with multiple transmitting LEDs system, where MRC is used to combine the signals received by the multiple photo-detectors. The BER is studied for the generalized multiuser considering OOK and perfect and imperfect SIC. Additionally, closed-form expressions are derived for the three-user case. Furthermore, Dixit $e t$ al. [125] consider a two-user case, where the near and far users are assigned OOK and $M_{n}$-PPM, respectively. The closedform BER expressions are derived considering imperfect SIC as well as perfect and imperfect CSI. Moreover, this system model is extended for $2 \times 2 \mathrm{MIMO}$ with repetition coding and MRC in [126]. The BER is analyzed for perfect and imperfect SIC. Also, dynamic field of view strategies are proposed to control the incident angle of the optical signal in order to improve the performance.

Reference [127] studies the non-linear behaviour of the LED in MIMO channels. To address this performance limiting factor, a pre-distorter is designed using singular value decomposition-based Chebyshev precoding, which is applicable for correlated-channels scenario and arbitrary number of users. Also, imperfect CSI is taken in consideration. Additionally, cognitive-radio inspired NOMA (CR-NOMA) power allocation policy is introduced to satisfy the user's quality of service (QoS) requirements. Upper bound BER expressions are derived for square $M_{n}$-QAM considering Gaussian residual interference.

The authors of [128] analyze the BER performance of a unique 1-bit feedback based beamforming scheme and novel SIC detection for a $2 \times 1$ multiple-input single-output (MISO) UL FSO-NOMA system in negative exponential fading channels. The beamformer gives higher weights to the antenna with the higher channel gain, while the SIC decoding order is based on the 1-bit feedback to inform the receiver about the instantaneous channel gain order. Hence, the signal with the 
TABLE XIII: Summary of the IRS and backscatter communication work.

\begin{tabular}{|c||c||c||c||c||c||c||c||c||c|}
\hline$[\#]$ & System & Metric & Antennas & Direction & Channel & $M$ & $N$ & Receiver & CSI \\
\hline \hline$[129]$ & IRS & BER & SISO & DL & Rayleigh & $4+2$ & 2 & Imperf. SIC & Perf. \\
\hline$[130]$ & IRS & PEP & SISO & DL & Rayleigh & $\forall M$ & 2 & Imperf. SIC & Perf. \\
\hline$[131]$ & Backscatter Comm & BER & SISO & UL & Nakagam- $m$ & 2 & 2 & Imperf. SIC & Perf. \\
\hline$[132]$ & Backscatter Comm & BER & SISO & PLNC & Exponential & 2 & 2 pairs & JMLD & Perf. \\
\hline
\end{tabular}

better channel gain is decoded first and the performance is improved compared to the conventional SIC which assumes fixed decoding order. Considering subcarrier intensity modulation BPSK, the exact closed-form instantaneous BER expressions are derived and asymptotic upper bound for the BER are presented. In addition, analytical framework is presented for the average BER. Furthermore, the impact of carrier synchronization errors, and the combined impact of atmospheric turbulence and pointing error are analyzed, and their average BER expressions are presented in integral-form.

\section{B. IRS and Backscatter Communication}

References [129], [130] study the performance of DL IRSassisted NOMA system considering imperfect SIC, a single antenna base station and single antenna users. Additionally, it is assumed that the direct link between the base station and the users is not available, where the IRS is divided into groups where each group is responsible for reflecting the signal to one user. Furthermore, perfect knowledge of channel phases is used to cancel the channels' phases using the IRS, and hence the reflected signals by the IRS metasurfaces will add constructively. The authors of [129] consider the two-user scenario where QPSK and BPSK are assigned for the near and far users, respectively. The PDF of the end-to-end channel based on Rayleigh fading, i.e. base station-IRS and IRS-user, is derived using the central limit theorem to approximate the sum of independent double Rayleigh random variables. However, this approximation is only accurate when large number of metasurface elements are considered. The BER expressions are presented in a single-integral form considering the moment generation function. Bariah et al. [130] present accurate BER approximations for a single metasurface element and multiple ( $>10)$ metasurface elements, where the PDF of the end-toend channel based on Rayleigh fading, i.e. base station-IRS and IRS-user, is derived. For instance, an approximation of the double Rayleigh distribution is used for the single metasurface case, while central limit theorem is used to approximate the end-to-end channel for the multiple $(>10)$ metasurface elements. The derived union bound of the PEPs provides tight approximation of the BER for two-user case with BPSK considering different numbers of metasurface elements.

The authors of [131] study the performance of an UL bistatic backscatter communication-aided NOMA for IoT sensors. Two backscatter nodes are co-located near the carrier emitter assuming interference free channels. The backscatter nodes operate in two modes, namely the EH mode and the active mode, in which performing intentional impedance mismatch at the antenna input, produces two reflection coefficients to modulate the carrier signal with BPSK. The Nakagami$m$ fading channel multiplexes the modulated BPSK symbols in the power domain using NOMA. The backscatter reader receives the NOMA symbol and performs SIC for detection. Closed-from BER expressions are derived by ignoring the phases of the channels between the backscatter nodes and the backscatter reader. Also, the channels are assumed to be ordered, however, order statistics are not used. Rather than considering double-integration with respect to the channels random variables, the sum and difference of the random variables are approximated by another Nakagami- $m$ random variables.

The authors of [132] study the BER performance of a backscatter-assisted NOMA with physical layer network coding (PLNC). The system model under study considers two clusters and two users per cluster, namely the near and far clusters which want to exchange data. Hence, PLNC is used, where in two time slots the near cluster pair and the far cluster pair transmit their data to the access point using UL NOMA. Once these symbols are decoded using JMLD, PLNC performs XORing and send the PLNC symbols using DL NOMA in the third time slot. Then the users in each cluster use SIC to detect the symbols. Upper bound BER expressions are derived for the end-to-end system considering BPSK and exponential channel. It is worthy to mention that the ambient backscatter is located near the far cluster pair and only used during the third time slot. A comprehensive summary for the NOMA work with IRS and backscatter communication is included in Table XIII.

\section{Cooperative Communication}

Through cooperative NOMA, strong channel users cooperate with weak channel users to improve their received SNR. After signal processing, the relaying node forwards the received NOMA signal to the destination. A basic structure of a DL cooperative NOMA with HD and FD relays is shown in Fig. 7 .

To get a clear view of the cooperative NOMA system, assume a single source, a single relay, and a single destination. Assuming a direct, but weak, link exists between the source and the destination. The NOMA packet is simulatenesouly received by the relay and the destination. The relay node utilizes SIC to detect and decode the destination signal. Moreover, the relay may adopt a signal forwarding policy based on the achieved SINR, ot it can simply forward the destination's signal without any constraint. For example, the relay forwards the destination's decoded signal only if the achieved SINR exceeds a predefined SINR; otherwise, no signal is transmitted. If the relay just decodes the received SC signal, and forwards the destination's detected singal as it is, the relay is termed as a DF. Similarly, if the relay detects the destination's signal and then amplifies it before forwarding, it is called an AF relay. Moreover, if the relay 


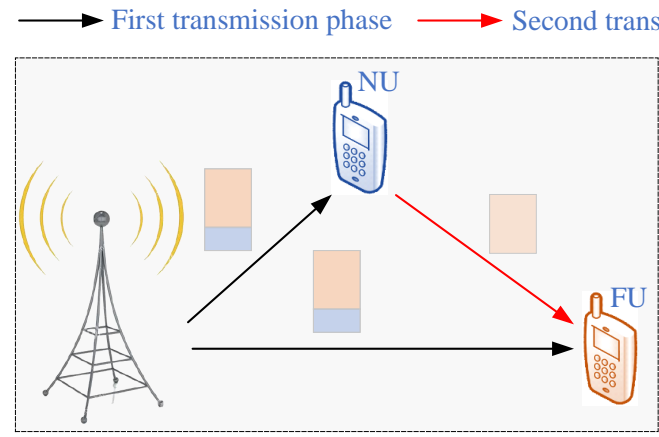

(a)

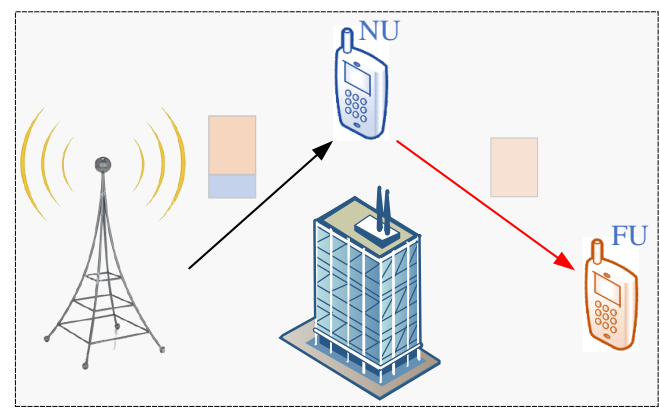

(c)
Amplified or Decoded signal for FU

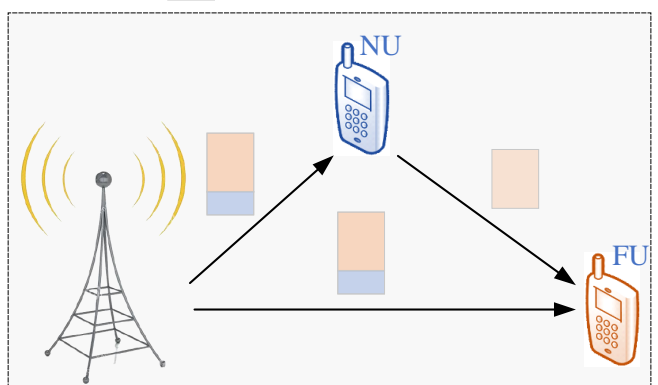

(b)

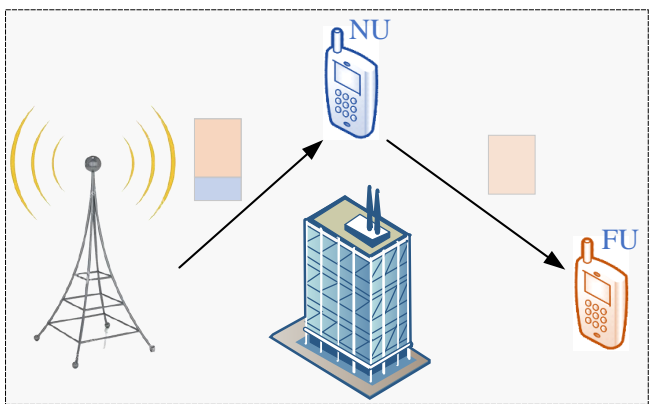

(d)

Fig. 7: Basic DL cooperative NOMA; (a) HD relaying and direct link, (b) FD relaying and direct link, (c) HD relaying without direct link, (d) FD relaying without direct link. NU can be a dedicated or a user relay.

receives and transmits in different time-slots, it is called a HD relay. Likewise, if a relay simultaneously receives and transmits in the same time-slot, it is known as a FD relay.

At the destination, the signal received from the source during the first phase is combined with the signal received from the relay node during the second phase. The signal can be combined using various techniques, e.g., MRC, selection combining $(\mathrm{SeC})$. Finally, the combined signal is passed through a MLD detector to estimate the signal at the destination. If no direct channel exists between the source and the destination, the only signal received by the destination is via the relay. In this case, the destination directly implements MLD to decode the received signal.

Occasionally, the relay will use the energy harvested from the received NOMA signal for cooperation [133]. The relay splits the received signal into two segments based on the splitting ratio, one for signal processing and the other for signal forwarding. If the relay node follows the AF protocol, it amplifies the received signal before forwarding; alternatively, if it follows the DF protocol, it simply decodes and re-encodes the signal before forwarding. Another cooperative NOMA with multiple relays and multiple destinations is provided in [134]. In this work, the relay with the best channel with the transmitter is chosen for cooperation.

a) The State-of-the-Art Research on Cooperative NOMA: Besides the fundamental cooperative NOMA schemes, considerable efforts have been devoted on developing enhanced cooperative NOMA systems for a wide range of applications. A comprehensive summary for the NOMA work with cooperative communication is included in Table XIV including the relying mode and the number of relays in the system. Moreover, a cooperative relaying system (CRS) with multiple relays assisting a single user, and a single relay assisting multiple users is shown in Fig. 8.

1) Conventional NOMA Cooperative Relaying System:

Dedicated relaying: Kara and Kaya [135], [136], consider a diamond-shaped CRS based on NOMA, with two HD-DF relays and a single destination. It is assumed that one relay is located distance from the source while the other relay is located closer to the source. The transmission is accomplished in two time slots, with the source transmitting SC of two BPSK modulated signals to both relays during the first time slot employing DL-NOMA. For decoding, the far relay employs MLD directly, whereas the near relay uses SIC. In the second phase, both relays use the UL-NOMA concept to forward the decoded information to the destination. Finally, the signal received from the near relay is detected by the destination, while the signal received from the distant relay is decoded using SIC. For both transmission phases, the authors have derived the average BER over SISO Rayleigh fading channels. Kara and Kaya [142] consider a two-user DL CRS with a single HD-DF relay located closer to the near user, where the direct link between the users and the BS does not exist. The PA in the first phase of communication is in the reversed order where the power coefficient of the near user is larger than the power coefficient of the far user. However, the PA in the second phase follows the conventional approach. It is worth noting that the SIC decoding order depends on the PA order. Given that the CSI is imperfect at the receiving nodes, the average BER is derived over Rayleigh fading channels 
TABLE XIV: Summary of the cooperative SISO NOMA work

\begin{tabular}{|c|c|c|c|c|c|c|c|c|c|c|}
\hline$[\#]$ & System & Mode & Metric & Direction & Channel & $M$ & $N$ & $R$ & Receiver & CSI \\
\hline [135], [136] & $\overline{\text { CRS }}$ & $\overline{\mathrm{H} \text { HD, DF }}$ & $\overline{\overline{\text { BER }}}$ & $\begin{array}{l}\text { DL,UL } \\
\end{array}$ & Rayleigh & 2 & 1 & $\overline{2}$ & $\begin{array}{l}\text { Imperf. SIC } \\
\end{array}$ & 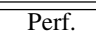 \\
\hline$[137]$ & CRS & HD,DF & BER & $\mathrm{DL}$ & Rayleigh & 4,16 & 2 & 1 & Imperf. SIC,MRC & Perf. \\
\hline [138] & CRS & HD,DF & BER & DL & Rayleigh & $4+2$ & 2 & 1 & Imperf. SIC,MRC & Stats. \\
\hline [139] & CRS & $\mathrm{HD}, \mathrm{AF}$ & PEP & DL & Rayleigh & 2 & $\forall N$ & 1 & Imperf. SIC & Perf. \\
\hline [140] & CRS & HD,DF & BER,PER & $\mathrm{DL}$ & Nakagami- $m$ & 2 & 4 & 2 & Imperf. SIC & Perf. \\
\hline [141] & CRS & $\mathrm{HD}, \mathrm{AF}$ & SER & UL & Rayleigh & 4 & 2 & 1 & Imperf. SIC & Perf. \\
\hline [142] & CRS & HD,DF & BER & DL & Rayleigh & 4 & 2 & 1 & Imperf. SIC & Imperf. \\
\hline [143] & CRS & FD,DF & PEP & $\mathrm{DL}$ & Rayleigh & 2 & 2 & 1 & Imperf. SIC & Perf. \\
\hline [144] & CRS & HD,DF & BER & DL & Nakagami- $m$ & $\leq 4$ & 1 & 1 & Imperf. SIC & Perf. \\
\hline [145] & CRS & HD,DF & BER & DL & Rayleigh & 2 & 2 & $R$ & Imperf. SIC & Perf. \\
\hline [133] & SWIPT & HD,AF/DF & PEP & DL & Rayleigh & 2 & 2 & 1 & Imperf. SIC & Perf. \\
\hline [146] & SWIPT & $\mathrm{HD}, \mathrm{AF}$ & PEP & DL & Rayleigh & 4 & $\forall N$ & 1 & Imperf. SIC & Perf. \\
\hline [147] & SWIPT & $\mathrm{HD}, \mathrm{AF}$ & PEP & DL & Rayleigh & 2 & $\forall N$ & $N$ & Imperf. SIC & Perf. \\
\hline [148] & SWIPT & $\mathrm{HD}, \mathrm{AF}$ & PEP & DL & Rayleigh & 2 & $\forall N$ & $R$ & Imperf. SIC & Perf. \\
\hline [149] & SWIPT & HD,DF & BER & $\mathrm{DL}$ & Nakagami- $m$ & 2 & 1 & 1 & Imperf. SIC & Perf. \\
\hline [134] & $\mathrm{CR}$ & $\mathrm{HD}, \mathrm{AF}$ & PEP & DL & Rayleigh & 4 & $\forall N$ & $R$ & Imperf. SIC & Perf. \\
\hline [150] & $\mathrm{CR}$ & HD,DF & BER & UL,DL & Rayleigh & 4 & 2 & 1 & Imperf. SIC & Perf. \\
\hline [151] & $\mathrm{CR}$ & HD,DF & BER & DL & Nakagami- $m$ & 2 & 2 & 1 & Imperf. SIC & Perf. \\
\hline$[152]$ & $\mathrm{CR}$ & HD,DF & BER & DL & Rayleigh & 2 & 2 & $R$ & Imperf. SIC & Perf. \\
\hline [153] & VLC & HD,DF & BER & DL & Non-fading & $2^{\star}$ & $\forall N$ & $N-1$ & MRC-Imperf. SIC & Perf. \\
\hline$[28]$ & VLC & AF-DF & BER & DL & Gamma-Gamma, Non-fading & 2 & 2 & 2 & Imperf. SIC & Perf. \\
\hline
\end{tabular}

considering QPSK for both users.

Kara et al. [144] propose using NOMA for a singleuser CRS to overcome the loss of spectral efficiency. In the proposed model, the source transmits a NOMA symbol, $\sqrt{\alpha_{1}} x_{1}+\sqrt{\alpha_{2}} x_{2}$, to the user and the HD-DF relay, where both detect $x_{2}$ using MLD, while only the relay detects $x_{1}$ using SIC. In the second phase, the relay forwards $x_{1}$ to the destination. The average BER expressions over Nakagami- $m$ fading channel are derived assuming BPSK symbols. Further, the authors propose a machine-learning PA algorithm to minimize the BER. The residual hardware impairments of a DL NOMA CRS that consists of $N$ users and a single HD-AF relay is studied in [139]. The users are assumed to be within close proximity of each other, while the link between the BS and the users does not exist. Assuming that imperfect SIC is used by the users, the union bound on the PEPs is derived over ordered Rayleigh fading channels to examine the system's BER. The results indicate that the residual hardware impairments have a significant effect on the cooperative NOMA system. A twouser DL NOMA CRS with $R$ HD-DF relays is investigated in [145], where each user has a dedicated relay that is selected based on max - min selection. Assuming the link between the BS and the users does not exist, the far-user, which is a highpriority user, applies MLD for detection, while the near-user, which is a delay-tolerant user, applies SIC for detection. The average BER expressions are derived over Rayleigh fading channel considering BPSK. In [141], a two-user UL NOMA CRS with a single HD-AF relay is studied, where both users are in the cell edge and the link with the BS does not exist. In the first phase, both users transmit their signal to the relay using UL NOMA principle, while in the second phase the relay applies linear noise reduction to the superposed signal, amplifies it with a gain factor, and finally transmits it to the BS. The BS executes SIC in order to separate the signals of two edge users. The authors have provided a theoretical analysis of the proposed system's performance in terms of SER. It is noted that at high SNRs the analytical and simulation results overlap.
User relaying: Kara et al. [137] studies the BER of a two-user DL cooperative NOMA system, where the nearuser acts as an opportunistic HD-DF relay for the far-user forwarding the decoded signal if the SINR is greater than a predefined threshold. Assuming that the direct link between the BS and the far use exists, MRC is used to combine the signals received from the BS and the relay, while MLD is used for detection. The closed-form BER expressions are derived for both users over SISO Rayleigh fading channels considering $M_{n}$-QAM. It is worth noting that the analysis for the near-user is done considering imperfect SIC. Furthermore, the optimum threshold value that minimizes the BER is derived. By relaxing the threshold condition for relaying, the work in [138] derives the BER expressions assuming QPSK and BPSK are used by the near- and far-user, respectively.

Jain et al. [140] study the performance of a DL NOMA CRS that consists of two cell edge users assisted with a HD-DF user relay for each user. The near-far problem is considered for the relays such that the relays do not interfere with each other. The close relay uses imperfect SIC for detection, while the far relay uses MLD. While assuming the link between the BS and the users does not exist, the only mean for communication with the users is through the relays. The authors derive the average BER over Nakagami- $m$ fading channel, while the instantaneous packet error rate (PER) expressions are presented. An analysis of two-user DL NOMA CRS is provided in [143], where the near-user acts as a FD-DF for the far-user which has no link with the BS except through the relay. The relay receives a NOMA signal from the BS for both users and the self-interference signal caused by the FD mode. Both users detect and decode their corresponding signals using NOMA principle. The authors analyze the PEP of the system for both users over Rayleigh fading channels assuming MLD at the far-user and imperfect SIC at the near-user. In addition, an asymptotic analysis is performed for both users at high SNRs. The analytical expressions are validated for the BPSK case. The simulation results indicate that self-interference has a varied effect on the PEP performance of near- and far-user. 


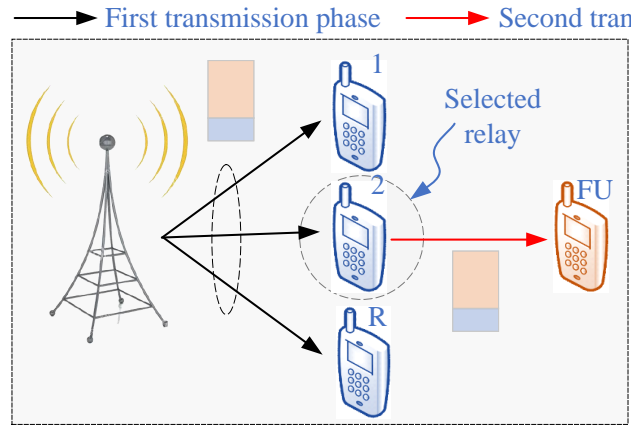

(a)

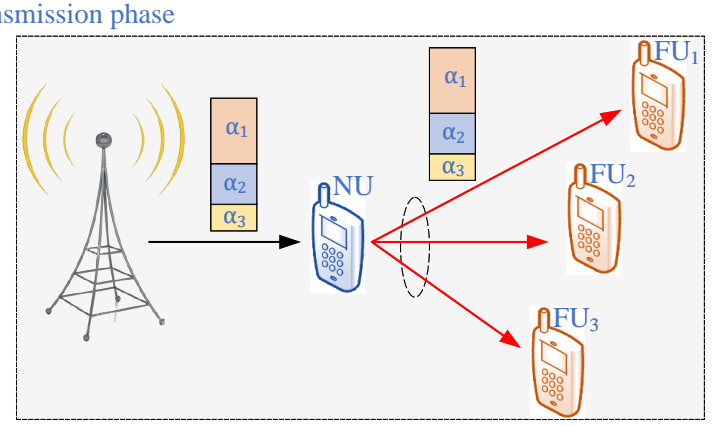

(b)

Fig. 8: CRS system; (a) Multiple relays assisting a single user, (b) A single relay assisting multiple users.

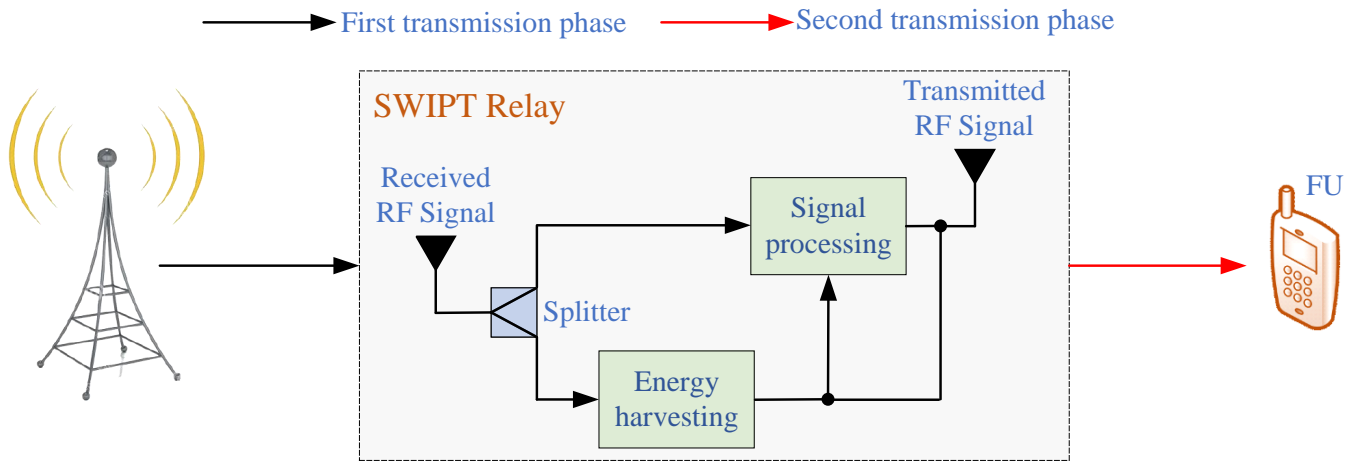

Fig. 9: Cooperative NOMA with SWIPT-enabled relay.

2) SWIPT-based CRS: Similar to the system model in [137], [138], the authors of [133] consider the near-user to be a SWIPT-enabled relay for the far-user, where the link between the BS and the far-user does not exist. The proposed model is shown in Fig. 9. The union bound on the PEPs over ordered Rayleigh fading channels is derived for the near- and far-user considering the AF and DF relaying protocols. It is worth noting that the analytical results are validated for the BPSK only. By performing asymptotic analysis on the PEPs, it is concluded that the maximum possible diversity orders for far- and near-user are one and two, respectively. Also, it is concluded that the splitting power ratio to enable SWIPT at the near-user may affect the whole cooperative NOMA system. Moreover, the authors of [146] extend the work to $N$ users, while assuming AF relay and Rayleigh fading channels with the large scale fading. The union bound on the PEPs is derived in closed-form. It is concluded that the maximum order of diversity is one, and the power splitting ratio and the position of the relay have a major effect on the system's performance. In [149], another cooperative NOMA system with SWIPT-enabled relay is investigated. The relay operates in HD mode and adheres to the DF protocol. Assume that all nodes have a single antenna and communicate through the Nakagami- $m$ fading channel. The relay harvest energy from the received SC signal during the first phase, and decodes the destination's signal using SIC. To obtain the system's end-toend exact closed-form expressions, BER analysis is performed. The average BER is derived using the number of incorrect bits between transmitted and detected symbols, as well as the total number of transmitted symbols. The analysis relies on the assumption of BPSK modulation. Due to the inherited interference of the NOMA, the conditional BER is determined by considering all possible combinations of the transmitted symbols. The average BER is then calculated by averaging the conditional BER over the channel gain. Simulation results are used to validate the analytical expressions.

In [147], a SISO cooperative DL NOMA for $R$ SWIPTenabled HD relays and $N$ users is proposed. Moreover, the relays adhere to the AF protocol. The relay harvest energy from the portion of received SC signal, whereas the residual signal is amplified and is transmitted to the users. Except the farthest user, all users implement SIC in order to detect their own signals. The PEP expressions are derived for all users. The simulations are performed for BPSK modulation with two users. Moreover, the accuracy of derived expression for the far-user is verified through simulations with BPSK, QPSK, and 16-QAM modulation. In [148], the cooperative NOMA is investigated where a single SWIPT-enabled relay from the total $R$ relays is selected during the broadcast phase for cooperation between the transmitter and $N$ users. The relay is selected based on the channel between the transmitter and the relay in question. Each relay is assumed to operate in HD mode, using the AF protocol. A fading channel following Rayleigh distribution is considered between all nodes. The selected relay harvests energy from a portion of the received signal and utilizes it to transmit the second portion of the signal to $N$ users following amplification. Then, for each user, SIC is used to determine its signal from the received NOMA signal. 
This work derives an exact PEP expression from an imprecise SIC assumption. Finally, using the derived PEP expression, an expression for the BER union bound is produced. For BPSK modulation, simulations are conducted.

3) CR-based Cooperative Relaying System: In [134], an underlying CR scenario in a SISO cooperative DL NOMA is presented. The system under consideration is composed of a primary and secondary BSs, and single primary and $N$ secondary users, which are serviced via $R$ AF relays. Moreover, a CR-based NOMA system is shown in Fig. Fig. 10. Transmission power constraints are enforced on the secondary BS and as single selected relay to maintain a tolerable level of interference for the primary network's reliable transmission. To obtain their individual signals, each user executes SIC on the received SC signal. For the proposed system, the authors have provided accurate PEP expressions. Following that, the derived PEP expressions are utilized to evaluate the BER union bounds. The system is simulated with QPSK modulation to verify the accuracy of the derived PEP expressions. Ustunbas et al. [150] proposes a cooperative NOMA-based CR butterfly network. The system is comprised of primary and secondary transmitters and receivers, and a single relay. The primary transmitter and secondary receiver share a cell, whereas the secondary transmitter and primary receiver share a cell. During the first time-slot, the QPSK modulated symbols are received simultaneously by the relay and the nearby receivers. The relay implements MLD to directly decode the secondary transmitter's signal, whereas the primary transmitter's signal is obtained through SIC. In the second time-slot, the relay generates a SC symbol from the detected symbols and forwards it to both receivers. Both receivers recover their respective symbols using SIC and MLD. The authors performed a BER analysis on the proposed system for both users using QPSK modulation and imperfect SIC assumption. The accuracy of the derived expressions is verified through simulation.

Another cooperative CR based SISO NOMA is presented in [152], where a BS serves a near- and a far-user. Due to absence of direct link between the BS and the far-user, the communication is established with the help of a single selected HD-AF relay among the $R$ available ones. The fading channel between the nodes is assumed to follow Rayleigh distribution. Moreover, it is assumed that perfect CSI is available to the relays. The SC signal is received by the relays and the near-user, where the near-user detects and decodes its own signal using SIC. Meanwhile, the relay with maximum SNR with the faruser is selected for cooperation. In the next round, the selected relay forwards the far-user's signal through controlled power to avoid interference to the near-user. The performance analysis of the proposed scheme is provided in terms of average BER. The proposed model is verified through simulations using BPSK modulation, and it is proved that the simulations and analytical results are almost identical. To preserve the nearuser's QoS, [151] proposes a cooperative spectrum sharing mechanism based on NOMA. It is assumed that the receivers have availability to perfect CSI. The system is composed of primary and secondary transmitters and receivers, where the secondary transmitters acts a DF relay. An SC signal is broadcast by the primary transmitted, which is simultaneously received by the primary receiver and the secondary transmitter. Both nodes perform SIC to remove interference due to primary transmitter's odd and even symbols. The secondary transmitter broadcasts its own signal and the detected even symbol of the primary transmitter using NOMA, which is received by the primary and secondary receivers. The secondary and primary receivers perform SIC and MRC-SIC, respectively, to decode their corresponding signals. The proposed system is analyzed in terms of average BER for BPSK modulation and imperfect SIC. It is concluded that the proposed scheme can provide desired QoS to the far-user while maintaining user fairness.

4) VLC-based Cooperative Relaying System: The proposed cooperative NOMA for a VLC application in [153] is composed of an LED transmitter and $N$ users. The LED broadcast the OOK modulated SC signal through a non-fading channel to $N$ users. The users performs SIC to decode the individual signals. In this way, the nearest user obtains the signals of all other users, while the farthest user will get its own signal only. The second round is composed of $N-1$ time-slots during which each user forwards the detected signals of the other users. This cooperation ensures the link reliability for the far users. Moreover, the far users implements MRC-SIC decode their desired signals. A performance analysis is done on the proposed model under perfect CSI and imperfect SIC assumptions to obtain the BER analytical expressions.

In [28], an optical backhauled cooperative NOMA with VLC system is presented. The FSO node transmits an optical signal in the proposed model. The PDF that describes the SNR of the FSO link takes into account the turbulence in the atmosphere, pointing errors, path loss, and AWGN. The relay node eliminates the direct current bias, but the remaining signal contains fading FSO irradiance variations and the photodiode's responsibility. The relay amplifies and biases the signal in order to drive it to the user. The signal is received by the indoor user following rectification of non-linear optical fiber impairments by an amplifier. The indoor user broadcast the SC signal for two users through a Gamma-Gamma, nonfading channel. Finally, the users perform SIC to decode their respective signals. The error-rate performance is evaluated in terms of user's average BER for BPSK modulation. The first step is to derive the expressions for the cumulative distribution function $(\mathrm{CDF})$ of the equivalent end-to-end SNR of both users. It is demonstrated through simulations that the proposed system may achieve a large performance gain when using a high-rate optical backhaul link with NOMA.

\section{Index Modulation}

1) Spatial modulation (SM): SM is recognized as the lowcomplexity and energy-efficient MIMO transmission scheme in the $5 \mathrm{G}$ cellular network. The information is transmitted in SM systems using both the index of an active antenna and a $M$-ary modulated symbol. The transmitted bit stream is partitioned into two groups, one of which is mapped to the activated transmitted antennas, and the other one is mapped to a conventional constellation and then transmitted via the activated antennas. When a single antenna is activated during a transmission, the system's complexity and energy usage are 


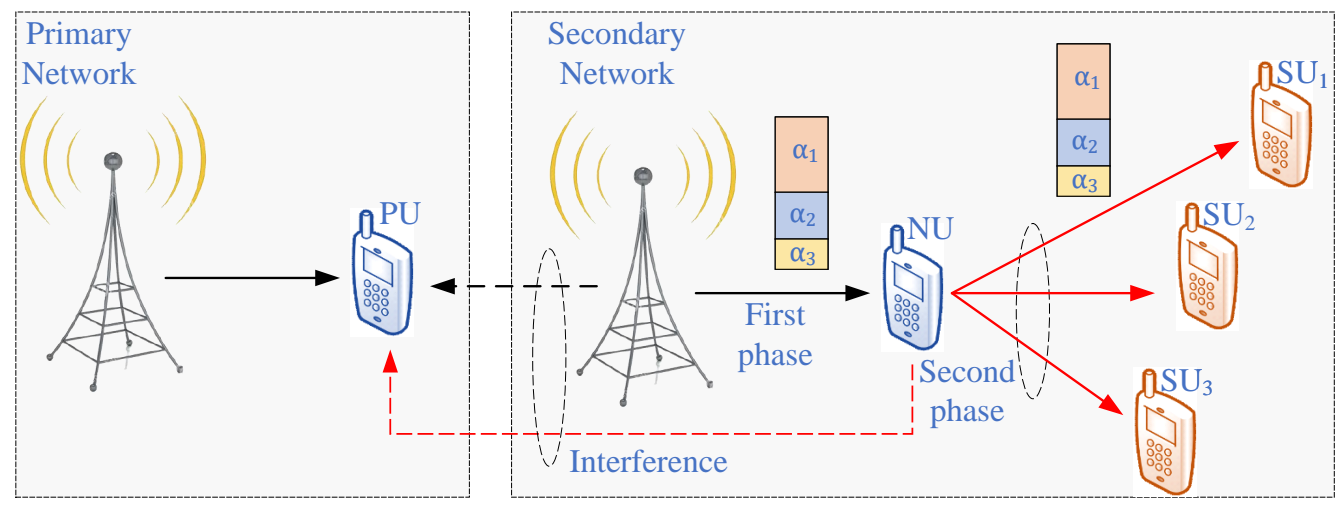

Fig. 10: A cognitive radio (CR)-based DL-NOMA proposed in [134].

reduced. Additionally, FD relaying is possible with the use of $\mathrm{SM}$, as one antenna of the relay can be used for transmission while the remaining antennas are utilized for reception.

By incorporating SM to NOMA systems, the spectral efficiency (SE) can be increased without increasing power consumption or implementation complexity. Additionally, the commonly used space division multiple access (SDMA) protocol suffers substantial IUI in today's multiuser SM systems. Given the effectiveness of NOMA in mitigating IUI, it appears to be a promising multiple access mechanism for SM-based multiuser communications. Thus, the combination of SM and NOMA is encouraged to capitalize on both SM and NOMA's potential benefits. To understand how SM-based cooperative NOMA works, assume a system with a single transmitter and two users, with the near-user acting as a relay for the faruser, as shown in Fig. 11. The transmitter, relay-user, and far-user each have multiple antennas. The whole transmission is divided into two phases if the relay follows HD protocol, whereas the transmission can be accomplished in a single round if the relay is operated in FD mode. During the first phase, the transmitter broadcasts the SM symbol, which is simultaneously received by the relay and the far-user. The SM symbol is given as $[0,0, \cdots, 0, s, 0, \cdots, 0,0]^{T}$, where the $s$ is the modulated symbol, while its location in the vector corresponds to the active antenna index. The relay node employs ML detection to decode estimated $\hat{s}$ the active antenna index. The relay node then recovers its own data bits from $\hat{s}$. The relay node then forwards the preserved active antenna index in the second phase. Following that, the faruser combines the signal transmitted by the relay-user during the second phase with the signal received from the transmitter during the first phase. Finally, the far-user applies the MLD to the combined signal to detect its own signal.

a) The State-of-the-Art Research on SM-NOMA: Conventional SM-NOMA: In [154], a DL MIMO cooperative NOMA with precoded spatial modulation (PSM) is presented. The system is comprised of a BS and 2 users. The proposed system employs an efficient power allocation mechanism to share all antennas for DL transmission. The PSM signal received by the users through a Rayleigh fading channel. Users implement SIC to mitigate IUI, while PSM detection is used to recover the bits. The authors conducted an analysis of

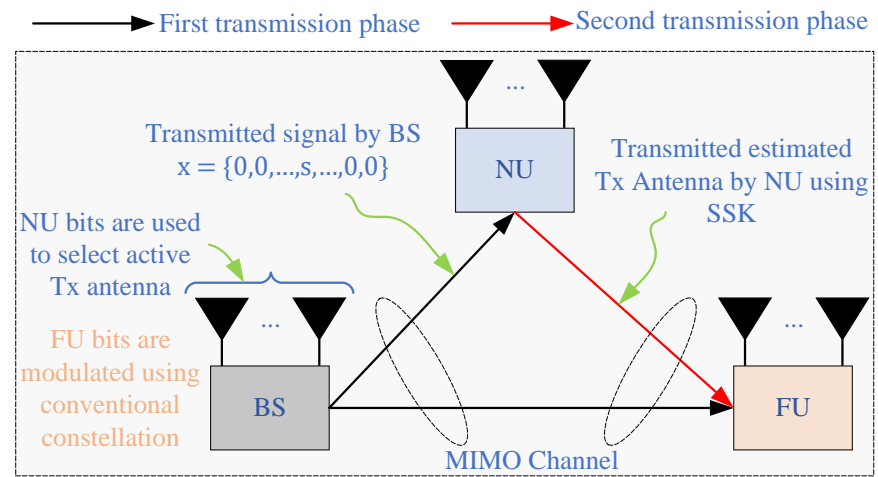

Fig. 11: SM-NOMA proposed in [163].

the proposed system in term of SER and MI. It is noticed from the resulting SER expression that there is a detection error propagation effect in SIC-MLD, where errors occurring in previously detected users influence subsequent users. The simulations demonstrate that the proposed model outperforms conventional designs in terms of capacity/SER. Another DL MIMO scenario with a BS and $N$ users is proposed in [155], [156]. The users divided are into multiple groups, with each group including two users. To avoid the inter-channel interference, the BS communicates with each group within orthogonal resource block. It is assumed that full CSI is available at the receivers, but not at the transmitter. According to SM, the bits of the first and second user in a group are modulated using $M$-ary and $s s k$, respectively, and an SM vector is generated. The first user in the group performs MRC-MLD to detect its own signal, while the information of the second user in a group is used to determine the index of transmitting antenna using an optimal MLD-based SM detection. The authors conducted a performance analysis of the proposed system in terms of average BER for both users in a group. The closed-form expression for average BER is obtained by averaging the conditional BER over instantaneous SNR. The simulation results demonstrated that the proposed model outperformed the standard NOMA system.

In [157], a generalised pre-coding quadrature spatial modulation (GPQSM)-NOMA is proposed. The proposed system consists of a BS and $N$ users, with the first user presumed 
TABLE XV: Summary of the conventional SM work.

\begin{tabular}{|c||c||c||c||c||c||c||c||c||c|}
\hline$[\#]$ & System & Metric & Antennas & Direction & Channel & $M$ & $N$ & Receiver & CSI \\
\hline \hline$[154]$ & Precoded SM & SER & MIMO & DL & Rayleigh & $\leq 4,8$ & 2 & Imperf. SIC & Perf. \\
\hline$[155]$ & SSK-NOMA & BER,PEP & MIMO & DL & Rayleigh & 4 & 3 & MRC-Imperf. SIC & Perf. \\
\hline$[156]$ & SSK-NOMA & BER,PEP & MIMO & DL & Rayleigh & $\leq 4$ & 2 & MRC-MLD & Imperf. \\
\hline$[157]$ & SM-NOMA & PEP & MIMO & DL & Rayleigh & 4 & $\forall N$ & Imperf. SIC & Perf. \\
\hline$[158]$ & SM-NOMA & BER & MISO & DL & Double Nakagami- $m$ & $\forall M$ & 2 & Imperf. SIC,MLD & Perf. \\
\hline$[159]$ & SM-NOMA & SER & MIMO & DL & Rayleigh & 4 & $\forall N$ & Imperf. SIC & Perf. \\
\hline
\end{tabular}

TABLE XVI: Summary of the SM with DL CRS work under perfect CSI conditions.

\begin{tabular}{|c||c||c||c||c||c||c||c||c|}
\hline$[\#]$ & Mode & Metric & Antennas & Channel & $M$ & $N$ & $R$ & Receiver \\
\hline \hline$[160]$ & FD,DF & PEP & MIMO & Rayleigh & 2,8 & 2 & 1 & MLD \\
\hline$[161]$ & HD,DF & BER & SISO & Rayleigh & 2 & 1 & $R$ & Imperf. SIC \\
\hline$[162]$ & HD,DF & PEP & MIMO & Rayleigh & $\leq 8$ & 2 & 1 & Imperf. SIC \\
\hline$[163]$ & HD,DF & PEP & MIMO & Rayleigh & $\forall M^{\bullet}, \forall M$ & 2 & 1 & MRC-JMLD \\
\hline$[164]$ & HD,DF & PEP & SIMO & Rayleigh & $2,4,16$ & $\forall N$ & 1 & Imperf. SIC \\
\hline
\end{tabular}

to be the nearest and the last one to be the farthest one. The BS generates the NOMA signal using the corresponding normalized powers and the zero-forcing pre-coding values. The receiver employs SIC to compensate for the MI effects inherent in the received signal. The signal of the farthest user is decoded directly using MLD, while SIC process is employed to decode the signals of other users. The authors propose a method for low-complexity detection and then derive an expression for PEP for the proposed method. The simulations demonstrate that by properly allocating power to the near- and the far-users, respectively, a relatively good and acceptable performance may be attained.

Jaiswal et al. [158] discusses a DL NOMA-based vehicle-tovehicle $(\mathrm{V} 2 \mathrm{~V})$ communication system. The vehicular system is composed of a source, and a near- and far-user, where all nodes operate in the HD mode. The proposed model considers SISO and MISO scenarios. Due to the vehicular nature of the nodes, this work considers an independent and non-identical double Nakagami- $m$ channel. The source broadcasts the SC signal through an opportunistically selected antenna. Both users detect and decode their desired signals using the conventional NOMA concept upon receiving the SC signal. The proposed system's performance is assessed in terms of average BER in the SISO and MISO cases. The exact expression for both users under SISO is derived. The infinite summation is presumed to be convergent, which makes it practical and tractable to employ. Finally, the total average BER is calculated utilizing the average BER expressions of both users. Further analysis of the system's average BER performance for the two proposed transmitting antenna selection strategies is conducted. Finally, numerical and simulation studies are used to validate the derived expressions.

In [159], a DL NOMA-SM is provided in which the BS simultaneously transmits the signals of multiple users via shared multiple transmitting antennas with varied optimal power allocations. To minimize IUI, a complicated SIC technique is employed at the receiver end to decode the signals. The proposed system is evaluated in terms of SER for all users using point-to-point phase shift keying (PSK) SM transmission over a Rayleigh fading channel. The derivation of SER takes into account the users' assumption of imperfect SIC. Using the resulting SER expressions, an optimal power allocation optimization problem is formulated to minimize the average SER for all users. Table XV provides a summary for the work in this part.

SM-NOMA with CRS: Si et al. [160] proposes a cooperative MIMO NOMA based on SM. The system consists of a BS, a FD relay, a near- and a far-user. The SM is used at both the BS and the relay. The relay receives and transmits concurrently because of SM. A portion of far-user's data is used to select the transmitting antenna, while the other data portion is applied to conventional constellation. Afterwards the BS broadcast the SC signal through the activated antenna. The SC signal is received by the near-user and the relay, where the near-user performs SIC to detect its signal while the relay preserves the signal till next time-slot. In the next time-slot, the relay simultaneously receives a new signal from the BS and forwards the former signal to the far-user, where it implements MLD to decode the received signal. Under Rayleigh fading, and perfect and imperfect SIC assumptions, the PEP closed-form expressions for both users are derived. Based on the results, it is concluded that the proposed system outperforms MIMO-NOMA with FD relaying and SM-OMA with HD relaying systems.

Li et al. [163] proposes a cooperative MIMO NOMA system with SM for a single BS and two users, where all nodes follow HD mode. During the first time slot, the BS broadcast the SM vector, where the information bits are modulated through conventional $M$-ary constellation. The transmitting antenna index is represented by the position of transmitting symbol in SM vector. The near-user implements MLD to obtain the transmitted symbol and the transmitting antenna index. Following signal estimation, the near-user recovers its own information from the estimated signal, whereas it preserves the antenna index for the far-user. During the second transmission round, the near-user forwards the preserved index to the faruser through an space shift keying (SSK) signal. The far-user combines both realizations and then recovers its signal using MLD. The authors have conducted a BER analysis on the proposed system. The proposed cooperative MIMO NOMA with SM system is compared to the conventional cooperative SISO NOMA with SM system. The simulation results show that the proposed system outperforms the conventional system in terms of BER. 
In [161], a distributed SM aided cooperative NOMA is proposed, in which the transmitter communicates with a single destination through a relay, selected among $R$ relays. The transmitter broadcasts an SC signal using a strong and weak signal. The relays performs SIC-MLD to detect the individual signals, whereas the destination only detects strong signal, while the detection of weak signal is postponed until the second time-slot. During the second time-slot, each relay compare the detected weak signal with its unique identity, and the relay whose identity matches the weak signal transfers data to the destination. The destination jointly detects the realizations received over two time-slots. The authors have proposed an error-aware MLD at the destination. The BER analysis of the proposed system with PSK/QAM modulation schemes is provided, and the proposed system is compared to a conventional distributed SM system in terms of BER through simulations. The results show that the proposed system outperform the conventional system.

In [162], a generalized quadrature SM with MIMO NOMA is investigated in a vehicular network. A subset of total antennas is chosen to transmit the modulated signal vector's real part, whereas the imaginary part is transmitted through the remaining antennas. The transmitted bits are divided into three groups, with one group being passed through a conventional modulator and the other two being used for antenna activation for the real and imaginary parts. The received SM signal is decoded through JMLD. The proposed system is analysed in terms of conditional PEP. The analysis is carried out using a simplified approximation of the complex Q-function. Further, the unconditional PEP is obtained using the conditional PEP, which is further used to determine the upper bound for the BER using the the union bounding technique. To assess the proposed system's practical applications, a cooperative NOMA generalized quadrature SM is investigated. The simulation results indicate that the proposed generalized quadrature SM outperforms the conventional generalized quadrature SM in the entire SNR region. Additionally, the cooperative NOMA generalized quadrature SM achieves a significant improvement of average BER over the cooperative OMA generalized quadrature SM.

Can et al. [164] investigates a CRS based DL NOMA with $N$ users and $R$ DF relays. The BS is equipped with single antenna, the relays are equipped with single receiving and multiple transmitting antennas, while the users are equipped with multiple receiving antennas. The BS employs the SMbased media-based modulation (MBM) technique through the use of a single antenna and multiple RF mirrors. Further, a perfect CSI is assumed to be available at the receivers. The transmitted bits for the users are partitioned into two groups. The bits in the first group are used to select active channel state, while the remaining bits are mapped to $M$-QAM constellation. The relay jointly decodes the active channel state index and the $M$-QAM symbol using MLD. In the second time-slot, the relay forwards the NOMA symbol to all users using multi-level QAM. The users performs SIC to decode their desired signals. The performance in terms of PEPs is analysed over Rayleigh fading channels. It is demonstrated that as the data rate increases, the proposed NOMA system outperforms the conventional NOMA system in terms of BER. Table XVI provides a summary for the work in this part.

2) IM-OFDM/time division multiple access (TDMA): In IM-OFDM system, the bits for each user are partitioned into two subblocks. Additionally, one subblock is passed through a conventional modulator to obtain the transmitted signal, and the other subblock is utilized to determine the indices of the OFDM subcarriers over which the modulated symbol will be transmitted. For each user, this operation is repeated. Moreover, data is transferred across a segment of neighboring OFDM subcarriers. If the user selects a subcarrier for transmission, a symbol is transferred; otherwise, a 0 is transmitted. Using power-domain NOMA, the symbols of distinct users on each subcarrier are supercoded. Each user performs MLD on its own subcarriers to detect its own symbols. Therefore, in this situation, IM is used in the frequency domain with OFDM, which is referred to as IM-OFDM.

Similarly, multiple users can submit data in discrete timeslots using IM-TDMA. Each user transmits a block of bits during their allotted time-slot. The overall block is broken into two sub-blocks; where one sub-block is used to determine the transmission time-slot, while the other sub-block is modulated using the conventional modulation scheme in use. As a result, the single non-zero element in the transmitted vector is denoted by the symbol $x_{n, t}$, the location of which in the transmitted vector corresponds to the modulated time-slot. Therefore, $x_{n, t}$ is the transmitted signal by the $n^{\text {th }}$ user during the $t^{\text {th }}$ transmission slot. The receiver uses the MLD to recover the individual signals transmitted by each user. Table XVII provides a comprehensive summary of the work considering IM-OFDM/TDMA.

a) The State-of-the-Art Research on NOMA-based IMOFDM: In [167], a SISO system model based on IM-OFDM is presented. The system consists of a transmitter, a near- and a far-user. The users share multiple subcarriers of an OFDM block using SC. Moreover, the available power is shared between the two users based on the channel conditions and the target rates. The data bits are conveyed by near-user's subcarriers, and therefore are modulated through conventional $M$-ary constellation. The indices of active subcarriers are transmitted by the signal carriers of the far-user. It is worth noting that the transmission uses only a portion of the total available carriers, and this partial subcarrier selection leads to increased power of active subcarriers. The far-user performs MLD on each subblock received to jointly estimate the data and active subcarrier indices. The near-user performs SIC to decode its own signal. The conditional PEP is evaluated for the far-user to determine average BER. Rayleigh fading channel, perfect CSI, and imperfect SIC are assumed. As the data information is independent from one block to another, conditional PEP is considered for a single subblock. Furthermore, unconditional PEP is calculated by averaging over the instantaneous channel. The lower bound for average BER for BPSK is obtained for the near-user. The simulation results show that IM-NOMA outperforms OFDM-NOMA operating on frequency selective channels.

An UL NOMA with IM is proposed for grant free access (GFA)-URLLC users in [168]. The system consists of a BS, 
TABLE XVII: Summary of the IM-OFDM/TDMA work with SISO configuration.

\begin{tabular}{|c|c|c|c|c|c|c|c|c|}
\hline [\#] & System & Metric & Antennas & Channel & $M$ & $N$ & Receiver & $\overline{\mathrm{CSI}}$ \\
\hline [165], [166] & CRS HD,DF & $\overline{\mathrm{PEP}}$ & $\overline{\mathrm{DL}}$ & Rayleigh & $\leq 16^{*}$ & 2 & Imperf. SIC & Perf. \\
\hline [167] & IM-OFDM & PEP & DL & Rayleigh & 2 & 2 & Imperf. SIC & \\
\hline [168] & GFA-ultra-reliable low latency communication (URLLC) & BER & $\mathrm{UL}$ & Rayleigh & 2 & 2 & MRC & \\
\hline [169] & IM-OFDMA & PEP & DL & Rayleigh & $\forall M^{*}$ & $\forall N$ & JMLD & \\
\hline [170] & IM-SC-FDMA & PEP & $\overline{\mathrm{UL}}$ & Rayleigh & 2 & 2 & JMLD,Imperf. SIC & \\
\hline [171] & IM-TDMA & PEP & $\overline{\mathrm{UL}}$ & Rayleigh & $\overline{\forall M^{*}, \forall M}$ & $\forall N$ & JMLD & \\
\hline [172] & CPI-SCMA & PEP & UL & Rayleigh & $\leq 4$ & $\forall N$ & JMLD & \\
\hline
\end{tabular}

a delay sensitive and a delay tolerance user. Moreover, the first user is allowed grant-free access, resulting in collisions between the two users. Therefore, to alleviate the adverse effects of collisions, the first and second users, respectively, are assigned OFDM and IM-OFDM. A portion of bits are mapped to standard $M$-ary constellation to get the frequency-domain signal for the first user, which is then transferred to timedomain and is delivered to the BS through the Rayleigh faded channel. The received SC signals in multiple transmissions are combined and decoded using MRC to improve the first user's SNR. The proposed transmission model is evaluated in terms of the first user's SINR and BER. The probability of a subcarrier being active or inactive is included when computing the average BER for the first user. If the subcarrier is active, the first user is affected by both the second user and the AWGN. When the subcarrier is inactive, the first user only experiences the AWGN. The closed-form BER expressions for BPSK and $M$-QAM modulations are derived for the first user.

Chen et al. [166] proposes a IM-OFDM based SISO cooperative NOMA comprised of a BS, a near- and a far-user. Each node operates in HD mode. During the first phase, the BS processes the information bits through IM-OFDM and broadcast them via an OFDM block. The OFDM is block is divided evenly into subblocks. The IM operation is done independently within subcarriers. A fraction of the bits in an arbitrary subblock are used to select active subcarriers from a pool of available ones, while the remaining bits are mapped to the conventional $M$-PSK and QAM constellations. The faruser preserves the received SC signal, while the near-user utilizes SIC to decode individual signals. In the next round, the near-user forwards the far-user's signal with full power after applying IM-OFDM. Finally, the far-user combines the two received realizations and followed by MLD. For both users, the authors have provided BER analysis. The BER is analyzed for the near-user when the index of the active subcarrier is correctly or wrongly decoded. As a result, the index error probability is evaluated first in order to characterize the BER. Finally, an upper bound is established for the BER.

In [169], a DL NOMA with IM is provided. The model is composed of a BS that offers services to $N$ users. As with a traditional OFDMA system, the available bandwidth is divided into multiple orthogonal subcarriers, where the adjacent subcarriers are pooled to serve a subset of $N$ users. For each user, the BS broadcasts a block of bits. These bits are split into two sections, where the first section of bits is passed through a conventional $M$-PSK modulator, while the second section is used to determine the subcarriers' subset for transmission. The modulated symbols of different users on the same subcarriers are superimposed, The users perform optimal JMLD to estimate the individual signals. On the proposed system, an average PEP analysis is performed, which is then utilized to establish an upper bound on the average BER. The simulation results are nearly identical to the analytical result. Moreover, the proposed model outperforms the conventional NOMA model by a significant margin.

Chen et al. [165] proposed a DL cooperative SISO NOMA based on an OFDM scenario. The system consists of a BS, a near-, and a far-user, where all nodes operate in HD mode. During the first transmission session, the BS transmits the information processed through IM-based OFDM to both users via an OFDM block. The transmitted OFDM block is divided into equal subblocks, and the subblocks are processed independently and identically. A portion of bits in a subblock are used to determine the subcarriers for transmission, whereas the remaining bits are applied to the conventional constellation to get information symbols for the near- and far-user. Low and high order modulation schemes are employed for the far- and near-users, respectively. The OFDM subblocks are concatenated and are applied to an inverse FFT, then cyclic prefix (CP) is appended, and finally parallel-to-series conversion is performed. The generated symbols are broadcast to both users, where the near-user performs SIC to detect the signals of both users. It preserves the far-user's signal for the cooperation. The far-user, on the other hand, retains the received SC signal. In the next round, the far-user decoded its desired signal using MRC-MLD. A BER-based performance analysis is provided for both users. Both analytical derivations and numerical simulations demonstrate that the proposed system outperforms the classical cooperative NOMA system by a significant margin.

Shahab et al. [170] propose an IM aided UL NOMA for massive machine type communication (mMTC). NOMA is used in conjunction with IM-single-carrier frequency division multiple access (SC-FDMA) to alleviate the limited allocation of subcarriers. The two users transmit their respective IMSC-FDMA signals to the BS with different power levels. The receiver at the BS performs JMLD to the composite signal it has received. The proposed model is subjected to a BER analysis. The upper bound on the BER performance is determined using the union bound on the PEP. The PEP analysis is performed on the JMLD and SIC-based detectors. The detailed analysis of BER is reserved for future work.

b) The State-of-the-Art Research on NOMA-based IMTDMA: An IM-NOMA technique for UL communication based on TDMA is proposed in [171]. A BS and $N$ users are included in the proposed model. The complete transmission 
TABLE XVIII: Summary of the DL SPC work.

\begin{tabular}{|c||c||c||c||c||c||c||c|}
\hline$[\#]$ & System & Metric & Antennas & Channel & $N$ & Receiver & CSI \\
\hline \hline$[173]$ & CRS & BLER & SISO & Rayleigh & 2 & MRC,SeC-Imperf. SIC & Perf. \\
\hline$[35]$ & SPC & BLER & SISO & AWGN & 2 & Imperf. SIC & Perf. \\
\hline$[174]$ & CR,SWIPT & BLER & SISO & Rayleigh & 2 & Imperf. SIC & Imperf. \\
\hline$[175]$ & SPC & BLER & SISO & Nakagami- $m$ & $\forall N$ & Imperf. SIC & Perf. \\
\hline$[176]$ & SPC & BLER & SISO & Rayleigh & 2 & Imperf. SIC & Perf. \\
\hline$[177]$ & SPC & BLER & MIMO & Nakagami- $m$ & 2 & Imperf. SIC & Stats. \\
\hline$[178]$ & SPC & BLER & SISO & Rayleigh & 2 & Imperf. SIC & Perf. \\
\hline$[179]$ & SPC & BLER & SISO & Rayleigh & 2 & Imperf. SIC & Perf. \\
\hline
\end{tabular}

time frame for UL is divided into equal orthogonal timeslots. Each user transmits a block of modulated bits at each time-slot. The block is then split up into two sub-blocks, where the first portion determines the transmission time-slot index, and the second one is passed through the QAM/PSK modulator. The BS receives an SC signal from all users sending concurrently during a common transmission slot. The BS uses the JMLD to estimate the individual signals. A PEP analysis of the proposed system is provided. The PDF is obtained and then integrated with the instantaneous PEP to obtain the unconditional PEP. Finally, by employing the tight union bound approach, the unconditional PEP is used to obtain the average BER. The simulation results reveal a considerable improvement of the proposed model over the conventional TDMA system. Lai et al. [172] proposes a codeword position index (CPI)-based sparse code multiple access (SCMA) in which each user's data is communicated via the employed codebook and the indices of the codeword positions. The proposed scheme complies to the NOMA principle in each time-slot. The analytical expression for the average BER is deduced using the conditional PEP approach. While deriving the conditional PEP expression, the authors considered Rayleigh fading channel. Finally, the average BER's upper bound is defined by averaging the conditional PEP. At high SNR, the proposed scheme outperforms the traditional SCMA in terms of error rate.

\section{E. Short Packet Communication}

According to Shannon's capacity theorem, as the block length approaches infinity, the likelihood of decoding error becomes zero [35]. As a result, the error probability can be significantly reduced by lowering the block length. This type of communication is referred to as SPC. For the time being, the error probability associated with SPC cannot be ignored. In NOMA networks, SPC is being researched in order to attain a lower error rate. Table XVIII provides a summary for the work in this part.

a) The State-of-the-Art Research on NOMA-based Short Packet Communication: In [173], a DL cooperative NOMA for SPC is presented, where the near-user acts as a HDDF relay for the far-user. During the first phase, the BS transmits an SC NOMA signal to both users over a flat Rayleigh fading channel. In the second phase, the near-user decodes the far-user's signal through SIC and relays it. The far-user combines the two received realization during two phases and combines them. The authors derive closed-form expressions for average BLER, where linear approximation is used to find the exact closed-form solution. The derivation involves two combining techniques, i.e., $\mathrm{SeC}$ and MRC. The obtained average BLER expression is suitable for very short packets, but fails to scale well when the packet length is increased considerably. To tackle this limitation, an asymptotic average BLER is computed. Given that the near-user average BLER for both systems is equal, it is concluded that the faruser in the proposed cooperative NOMA achieves a lower average BLER than the standard NOMA. A DL SISO NOMA system with two users and a BS is considered in [35]. The BS broadcasts an SC signal which is directly decoded by the far-user using MLD, while the near-user applies SIC to decode both signals. Finally, it decodes the signal that has been detected. The authors evaluated the error probabilities for coded and uncoded systems for both users, considering QPSK modulation. The far-user's PDF and cumulative PDF are obtained. Due to the imperfect SIC assumption, the error probability for the near-user is tricky. For the coded system, the error probability is a function of SINR, block length, and target rate. In both cases, an optimal power allocation problem is proposed with the objective of minimizing the error probability. Finally, simulations are performed to assess the proposed model's performance under a range of fixed channel gains.

In [174], a DL wireless-powered CR-NOMA IoT network with SPC is presented. A BS, a secondary source node, two NOMA and primary users comprise the system. The secondary node transmits information to the near- and far- user using the energy harvested from the BS. All nodes, excluding the BS, are assumed to be equipped with a single antenna and to be operating in HD mode. The secondary source adjusts its transmission power to meet the QoS requirements for primary users. The first phase involves the $\mathrm{EH}$, while the next one is utilized for information transmission to both users through Rayleigh fading channel using the NOMA principle. The instantaneous BLER of the users represents the error bound, which is the function of SINR, Shannon capacity, the number of information bits, and the packet length. By integrating the instantaneous BLER, the system is further analyzed in terms of average BLER for both users. To get the exact closed-form expression, the $\mathrm{CDF}$ is derived using a linear approximation of the instantaneous BLER expression for SPC to deal with the complicated Q-function. Finally, the concept of first order Riemann integral approximation is used to handle the multiple integration involved in the CDF. Finally, the simulation results are compared to the analytical solution and to the results obtained using the deep neural network technique. A SISO 
TABLE XIX: Summary of the code domain NOMA work.

\begin{tabular}{|c||c||c||c||c||c||c||c||c||c|}
\hline$[\#]$ & System & Metric & Antennas & Direction & Channel & $M$ & $N$ & Receiver & CSI \\
\hline \hline$[180]$ & MUSA & BER & SISO & UL & AWGN & 4 & $\forall N$ & Imperf. SIC & Perf. \\
\hline$[181]$ & SCMA & PEP & SISO & UL & Rayleigh & $\leq 4$ & $\forall N$ & JMLD & Perf. \\
\hline$[182]$ & SCMA & PEP & MIMO & UL & Rayleigh & 4 & $\forall N$ & JMLD & Perf. \\
\hline$[183]$ & SCMA & BER & SISO & DL & AWGN & $\leq 16$ & $\forall N$ & MAP & Perf. \\
\hline$[184]$ & SCMA & BER & SISO & DL & Rayleigh & $\leq 16$ & $\forall N$ & MAP & Perf. \\
\hline
\end{tabular}

DL NOMA with SPC is investigated in [175]. A single BS delivers information bits to each of $N$ users over a Nakagami$m$ channel. To detect these signals, the receivers employ SIC. The conditional BLER is a function of the accomplished SINR, the total amount of data to transfer, and the blocklength for a user. The work is based on a perfect SIC assumption. The authors derived analytical expressions for the average BLER by utilizing the conditional BLER and the PDF. A linear function is used to approximate the complex integration required for the derivation. The $\mathrm{CDF}$ is constructed using the derived average BLER. Finally, simulations are performed to verify that the analytical results match the simulations exactly.

A two users DL NOMA with SPC is investigated in [176]. This work assumes a Rayleigh fading channel between the BS and the users. It is demonstrated that the blocklength can be represented as the users' power allocation coefficients. The authors developed an optimization problem in which the objective is to minimize the blocklength, which is bound by average BLER. As a result, a closed-form expression for average BLER is derived to solve the optimization problem and to determine the best minimum blocklength for each user. The average BLER is obtained by averaging the instantaneous BLER and the PDF. Finally, simulations are used to verify the theoretical analysis. Yuan et al. [177] proposes a MISO DL NOMA system for SPC with a Nakagami- $m$ channel between the nodes. Moreover, it is assumed that the channel remains constant across a two-symbol period. The instantaneous BLER is approximated as a function of the number of data bits, the packet length, and the attained SINR for perfect and imperfect SIC assumptions. Closed-form BLER expressions are derived for both users using the instantaneous BLER and the corresponding PDFs. Again, linear approximations are used to simplify the complicated integrals involved in the derivation. Finally, in the high SNR region, the asymptotic average BLER expressions are derived to obtain the expressions for the minimum blocklengths for both users. Numerical simulations are used to validate the analytical expressions.

A secure DL NOMA for SPC between a BS and two users is investigated in [178]. It is assumed that the faruser can eavesdrop on the signal transmitted to the nearuser. Users have access to the CSI, whereas the BS can exploit the channel reciprocity property to gain access to the CSI. The near user uses SIC to decode both individual signals, while the far-user directly decodes the SC signal using MLD. The average secure BLER of the near-user for detecting and decoding the signal of the far-user is derived in this study by considering both perfect and imperfect SIC assumptions. To simplify the analysis, the authors employed linear approximations. Finally, an asymptotic average secure BLER is constructed for the near-user in the high SNR region.
In [179], a hybrid DL NOMA is proposed, in which the nearuser requests a long packet while the far-user requests an SPC. Between the nodes, a flat Rayleigh fading channel is assumed. The authors analyzed the proposed model in terms of average BLER for both users. The analytical formulae are derived from the outage probabilities. Yet again, linear techniques are used to simplify the analysis. The simulations demonstrate that, with the same target average BLER of the far-user, the proposed model achieves a considerably lower outage probability than the OMA system.

\section{F. Code domain}

1) SCMA: SCMA is a recently proposed multi-carrier NOMA scheme to serve multiple users simultaneously by employing different codebooks. The information bits of each user in the SCMA aided NOMA system is mapped to multidimenesional complex codewords using a predefined codebook. Table XIX provides a summary for the work in this part.

a) The State-of-the-Art Research on NOMA-based SCMA: Using non-orthogonal spreading sequences, multipleuser shared access (MUSA) is proposed for the UL GFA access in [180]. The power allocation is optimized to minimize the overall system's BER under two constraints: 1) total power constraint, and 2) minimum difference in received power between strong and interfering signals. At the receiver, SIC is employed in conjunction with the linear minimum mean square error (MMSE) detector. In [181], authors derive PEP closed-form expressions for joint ML under Rayleigh fading channel. The PEP is initially derived for a single user using a multidimensional constellation. The PEP expression is derived by comparing the transmitted and received vectors dimensionwise. Additionally, the single-user scenario is extended to obtain the PEP for JMLD in a multi-user scenario. Finally, the average SER upper bound is obtained using the unionbound technique and validated using simulations of the SCMA codebook.

In [182], a single-cell UL multiple-access communication system is presented. Each user activates the transmit antenna in the proposed model by using the SSK symbol. The remaining bits are applied to conventional modulation and then sent using SM through the activated antenna. At the receiver, the authors provide three different detectors: the optimal MLD, the optimal maximum apostiori detector, and finally a low-complexity near-optimal message passing algorithm for detection. The PEP performance analysis is conducted for the proposed system. A PEP is calculated utilizing both the transmitted and received vectors. Finally, the average BER from union-bound is obtained. In [185], an impulsive noiseimpaired DL SCMA is provided. The binary bitstream is 
converted to codewords, which are then superimposed to create the SC signal and broadcast through wireless channel. By invoking the concept of generalized SNR, the proposed communication model is analyzed in terms of BER. Reference [183] models a DL SCMA system with $N$ users. To avoid IUI, the receiver employs a message passing algorithm. The star-QAM is considered in the BER analysis, and the decision region is defined to minimize the BER.

Furthermore, [184] discusses in detail the BER analysis of the SCMA codebook generated using star-QAM. The codewords' phases are uniformly distributed throughout the constellation. The CDF is derived using the phase difference between the transmitted and received codewords. Finally, the proposed system's average BER is derived over a Rayleigh fading channel in terms of the probability that the received symbols fall inside the decision region of the transmitted codeword and the Hamming weight of the bits associated with a particular symbol. Simulations are used to validate the derived analytical BER expressions. Ling et al. [186] propose a multiple decision SIC for the UL code-domain NOMA to mitigate the error propagation problem of the conventional SIC, where multiple codeword candidates are considered rather than one. A tradeoff between complexity and performance is achieved by selecting candidates that have probabilities greater than a predefined threshold.

\section{ERROR RATE LITERATURE ClassiFICATION}

This section aims to highlight the different NOMA system model traits considered by the researchers. These traits are classified into six categories which are:

- Antennas setup

- Number of users

- Error performance metric

- Modulation scheme

- Channel model

- Receiver

Fig. 12 illustrates the different categories in a snowflake diagram which counts the number of papers that consider a certain trait for each category. This looks like a compass to guide the readers to the most common assumptions made in the research community for NOMA systems. It is worthy to mention that the scale is logarithmic in the figure.

It can be seen in Fig. 12a, that SISO is the mostly considered antennas setup by researchers, while MISO is the least considered. This is because MISO-NOMA beamforming requires optimization which makes it challenging to derive closedform expressions for the error performance metrics. Hence, it can be seen as a promising direction for NOMA error rate analysis research. Furthermore, as can be noted from Fig. 12b two-user NOMA system is mostly considered by researchers to minimize the receiver complexity since the complexity of the SIC chain increases when more users are assumed. Interestingly, arbitrary number of users is also considered by some researchers to verify the performance of the NOMA system in extremely overloaded scenarios.

The BER is exhaustively studied by researcher to validate the error performance of NOMA according to Fig. 12c, whereas BLER is the least studied. In addition, the PEP analysis has been considered widely to evaluate the NOMA system performance when the BER and SER analysis are difficult to obtain or intractable. The statistics show that the BLER, which is mostly considered for SPC, did not receive remarkable attention by the research community compared to other error rate metrics. Hence, it can be seen as a promising direction for study. Further, Fig. 12d shows that QAM is used very frequently in the literature due to its energy packing capability and energy efficiency. The other modulation schemes such as OOK and PSK are mostly used by VLC systems which received significant attention.

Fig. 12e shows that Rayleigh fading channel is the mostly considered channel in the literature since it is widely accepted for modeling the non-LoS wireless channel. The second mostly adopted channel model is the AWGN channel, which is commonly accepted to derive the closed-form instantaneous error rate performance. While deriving the average error rate performance might be challenging for UL NOMA systems, it is accepted to consider mathematical approximation or single integral-form expressions. It is worthy to mention that channel models such as Nakagami- $m$ and other generalized channel models can capture several models by tuning the channel parameters, which gives better insights about the system performance in different scenarios. However, deriving the average error rate performance becomes challenging. Furthermore, Fig. 12f shows that imperfect SIC receiver assumption is dominating over the other receiver assumptions. The reason is that the such a receiver captures the error propagation issue faced in practical scenarios. In addition, its complexity is much lower compared to the JMLD. It is worthy to mention that MRC is used mostly to combine the transmitting or receiving diversity signals. Many researchers focused on enhancing the receiver which is under others trait.

\section{Future Directions AND OPEN RESEARCH PROBLEMS}

This section aims to highlight future directions and open research problems of NOMA systems, while focusing on the error rate performance.

\section{A. Hybrid IRS}

As discussed in Sec. II, IRS can enhance the system performance by controlling the signals propagation in the environment via passive smart metasurface elements. However, there are several challenges such as acquiring channel estimates between all metasurface elements and users. Since the IRS is passive, it cannot transmit pilot/training signals to facilitate channel estimation. Hence, new channel estimation methods should be considered for the IRS such as estimating the cascaded user-IRS-BS channels, or by considering semipassive IRS models which have sensing capabilities [187]. Also, the phase cannot be controlled continuously in practice. Therefore, it is crucial to consider quantized phase control.

The error rate performance of IRS-assisted NOMA system has been investigated for the DL direction only, where the assumptions of continuous and perfect phase is assumed [129], 


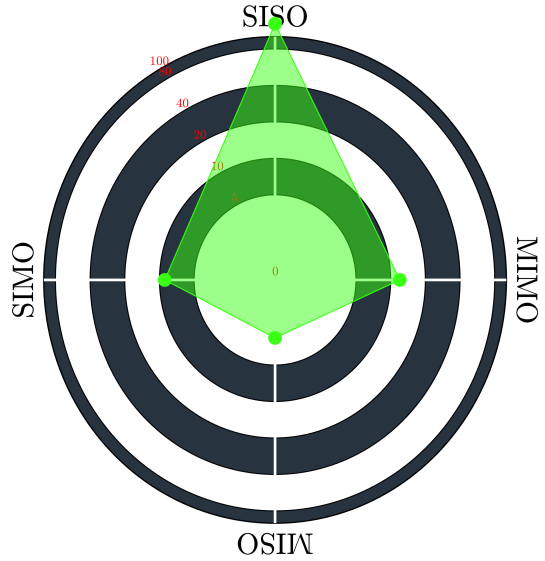

(a) Antennas Setup

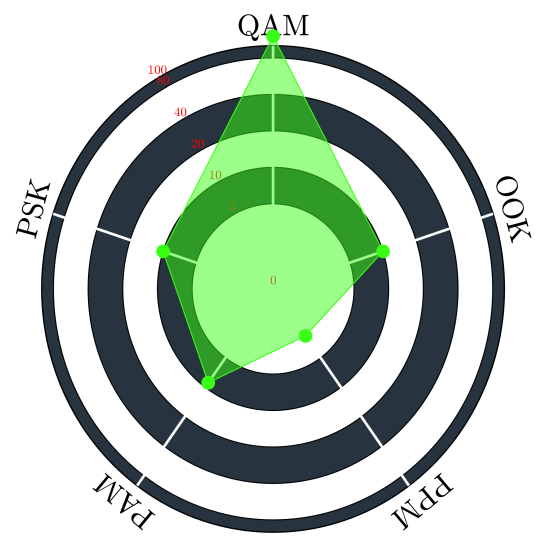

(d) Modulation Scheme

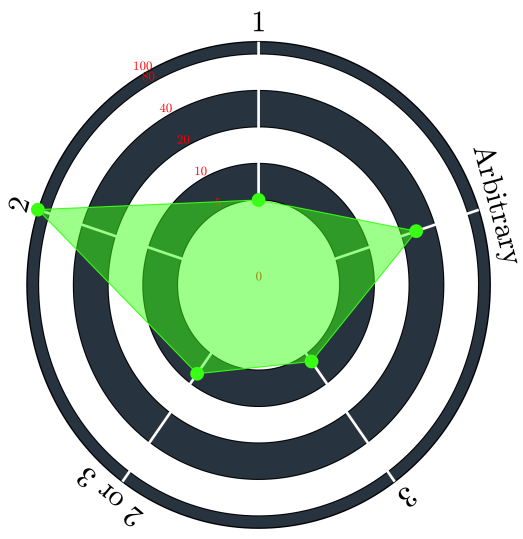

(b) Number of Users

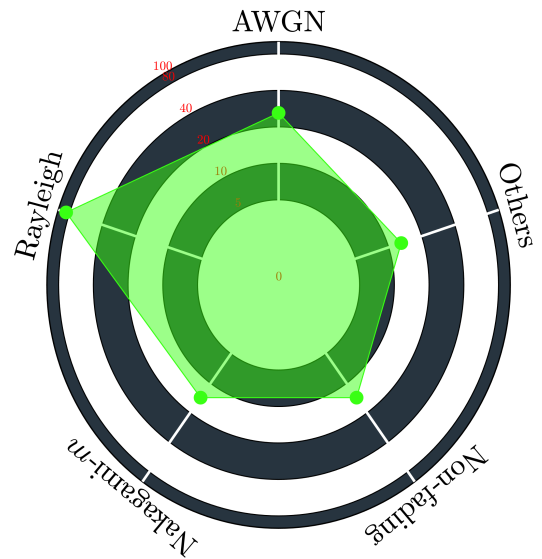

(e) Channel Model

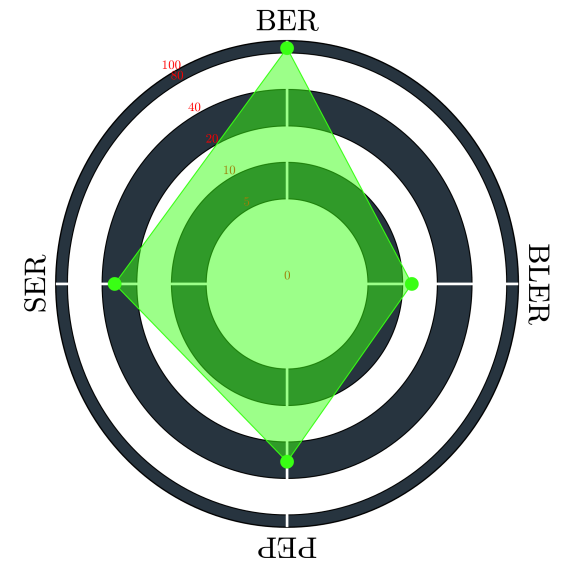

(c) Error Performance Metric

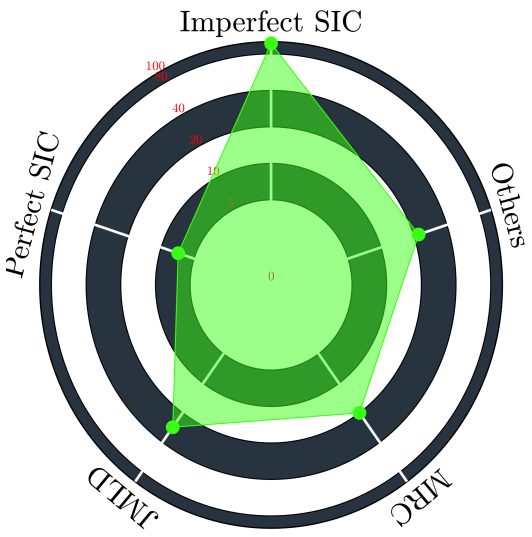

(f) Receiver

Fig. 12: Error rate work classification counter based on: (a) Antennas setup. (b) Number of users. (c) Error performance metric. (d) Modulation scheme. (e) Channel model. (f) Receiver.

[130]. Hence, analysing the error rate performance under imperfect and quantized phase is worth studying to get better practical insights. Moreover, the design and analysis for the UL direction is a promising direction as well. Furthermore, studying the system's performance while considering the direct link between the users and the BS is still an open research question. Another interesting direction is the simultaneous transmitting and reflecting IRS, where besides reflection, the IRS can function is a passive transmitter. This can further enhance the system's performance [188]. Hence, the design and analysis of the dual function IRS in DL and UL NOMA systems is attractive.

\section{B. Joint Radar-Communication Systems}

Joint radar-communication system may solve the spectrum scarcity problem as a single BS will perform the communication and sensing functions using the same frequency band. Hence, the beamformer will be optimized to satisfy the requirements for both functions. For instance, certain error rate performance or sum rate requirements for the communication part, while certain sensing power for the sensing part. While
NOMA is used to serve multiple users, it can be used for sensing simultaneously [189]. Investigating the error performance of the communication part and the effectiveness of the sensing part of the NOMA-based joint radar-communication system while considering proper signalling is still an open research problem.

\section{C. mmWave and $\mathrm{THz}$ Communication}

Higher frequency bands such as millimeter wave (mmWave) bands are currently used in the $5 \mathrm{G}$ standard to satisfy the growth in higher data requirements since these bands provide wider bandwidth. However, the mmWave bands will not be enough to support beyond $5 \mathrm{G}$ networks. Hence, terahertz $(\mathrm{THz})$ bands are wider and considered a key technology for future wireless networks [6]. Nonetheless, the propagation requirement would become more challenging and the communication would be directional and take place mostly in LoS environments. Therefore, the near-far problem would exist even if the users are relatively in close proximity. Consequently, studying the best conditions for pairing the NOMA users under certain error rate performance requirements can provide better 
understanding for the practical scenarios in which NOMA performance would be maximized. Furthermore, including technologies such as cooperative communication and IRS can be employed to improve the network coverage.

\section{Low Complexity Detectors}

The most crucial concern for deploying NOMA on a more than two-user per resource block scale is the detectors extensive complexity. While either SIC or JMLD computational complexities might limit the applications of NOMA, designing new detectors with lower complexity for the UL or DL is a promising research direction. Most importantly is the UL direction since JMLD is introduced as a solution for the SIC error floor problem [68]. Since JMLD has a relatively higher complexity compared to the SIC, the aim should be to design a detector that has a lower complexity while maintaining an acceptable performance.

\section{E. Imperfections in OFDM-NOMA Systems}

The work of OFDM-NOMA systems reported in this survey mostly assumes ideal conditions in terms of time and frequency synchronization, as well as CP length which is assumed to be longer than the channel delay spread, and hence the inter-symbol interference is removed perfectly. However, these ideal conditions might not be the case for practical scenarios where imperfect time and frequency synchronization is likely to happen. Additionally, the OFDM-NOMA is prone to user mobility which introduces Doppler shift and loss of subcarriers orthogonality. Therefore, studying the error rate performance of OFDM-NOMA under the previously mentioned imperfections is still an open research problem.

\section{CONCLUSIONS}

To summarize, this article surveys the state-of-the-art work in the literature of NOMA focusing on the papers that demonstrate analytical tools for the error rate performance. A comprehensive summary and tutorial is provided for the conventional NOMA scheme in the DL and UL directions. Also, improved NOMA schemes are highlighted including labeling, constellation rotation, interference alignment, channel coding multicarrier NOMA system design and detector design. Furthermore, a comprehensive survey is provided about the integration of NOMA and other technologies such optical communication, IRS and backscatter communication, cooperative communications, IM, SPC and code domain. Finally, the future research directions and the open NOMA research problems are identified.

\section{APPENDIX I: ACRONYMS}

\section{Notation Description \\ $M_{n}$-PAM $\quad M$-ary pulse amplitude modulation \\ $M_{n}$-PPM $\quad M$-ary pulse position modulation \\ $M_{n}$-PSK $\quad M$-ary phase shift keying}

\begin{tabular}{|c|c|}
\hline Notation & Description \\
\hline$M_{n}-\mathrm{QAM}$ & $\begin{array}{l}M \text {-ary quadrature amplitude modula- } \\
\text { tion }\end{array}$ \\
\hline $4 G$ & fourth generation \\
\hline $5 \mathrm{G}$ & fifth generation \\
\hline $\mathrm{AF}$ & amplify-and-forward \\
\hline ASK & amplitude shift keying \\
\hline AWGN & additive white Gaussian noise \\
\hline BER & bit error rate \\
\hline BLER & block error rate \\
\hline BPSK & binary phase shift keying \\
\hline BS & base station \\
\hline $\mathrm{CDF}$ & cumulative distribution function \\
\hline CDMA & code division multiple access \\
\hline $\mathrm{CP}$ & cyclic prefix \\
\hline CPI & codeword position index \\
\hline CR & cognitive radio \\
\hline CR-NOMA & cognitive-radio inspired NOMA \\
\hline CRS & cooperative relaying system \\
\hline CSI & channel state information \\
\hline DF & decode-and-forward \\
\hline DFT & discrete Fourier transform \\
\hline $\mathrm{DL}$ & downlink \\
\hline DWT & discrete wavelet transform \\
\hline ED & Euclidean distance \\
\hline $\mathrm{EH}$ & energy harvesting \\
\hline FD & full-duplex \\
\hline FEC & forward error correcting \\
\hline FFT & fast Fourier transform \\
\hline FSO & free space optical \\
\hline GFA & grant free access \\
\hline GFDMA & $\begin{array}{l}\text { generalized frequency division multi- } \\
\text { ple access }\end{array}$ \\
\hline GGN & generalized Gaussian noise \\
\hline GPQSM & $\begin{array}{l}\text { generalised pre-coding quadrature } \\
\text { spatial modulation }\end{array}$ \\
\hline HD & half-duplex \\
\hline IM & index modulation \\
\hline IoT & Internet of Things \\
\hline IRS & intelligent reflecting surface \\
\hline IUI & inter-user interference \\
\hline
\end{tabular}

JMLD joint-multiuser maximum likelihood detector

LDPC low-density parity-check 


\begin{tabular}{|c|c|}
\hline Notation & Description \\
\hline LED & light emitting diode \\
\hline LLR & log-likelihood ratio \\
\hline LoS & line-of-sight \\
\hline MAP & maximum a posterior \\
\hline MBM & media-based modulation \\
\hline MI & mutual information \\
\hline MIMO & multiple-input multiple-output \\
\hline MISO & multiple-input single-output \\
\hline ML & maximum likelihood \\
\hline MLD & maximum likelihood detector \\
\hline MMSE & minimum mean square error \\
\hline mMTC & $\begin{array}{l}\text { massive machine type communica- } \\
\text { tion }\end{array}$ \\
\hline mmWave & millimeter wave \\
\hline MPPM & multiple pulse position modulation \\
\hline MRC & maximal ratio combining \\
\hline MUSA & multiple-user shared access \\
\hline NOMA & non-orthogonal multiple access \\
\hline O-NOMA & optical-NOMA \\
\hline OFDM & $\begin{array}{l}\text { orthogonal frequency division multi- } \\
\text { plexing }\end{array}$ \\
\hline OFDMA & $\begin{array}{l}\text { orthogonal frequency division multi- } \\
\text { ple access }\end{array}$ \\
\hline OMA & orthogonal multiple access \\
\hline OOK & on-off keying \\
\hline PA & power assignment \\
\hline PAM & pulse amplitude modulation \\
\hline PCB & power coefficient bound \\
\hline PDF & probability density function \\
\hline PEP & pairwise error probability \\
\hline PER & packet error rate \\
\hline PLNC & physical layer network coding \\
\hline PMF & probability mass function \\
\hline PSK & phase shift keying \\
\hline PSM & precoded spatial modulation \\
\hline QAM & quadrature amplitude modulation \\
\hline QoS & quality of service \\
\hline QPSK & quadrature-phase shift keying \\
\hline $\mathrm{RF}$ & radio frequency \\
\hline $\mathrm{SC}$ & superposition coding \\
\hline SC-FDMA & $\begin{array}{l}\text { single-carrier frequency division mul- } \\
\text { tiple access }\end{array}$ \\
\hline SCMA & sparse code multiple access \\
\hline SDMA & space division multiple access \\
\hline SE & spectral efficiency \\
\hline $\mathrm{SeC}$ & selection combining \\
\hline SER & symbol error rate \\
\hline
\end{tabular}

$\begin{array}{ll}\text { Notation } & \text { Description } \\ \text { SIC } & \text { successive interference cancellation } \\ \text { SIMO } & \text { single-input-multiple-output } \\ \text { SINR } & \text { signal-to-interference-plus-noise ratio } \\ \text { SISO } & \text { single-input-single-output } \\ \text { SM } & \text { spatial modulation } \\ \text { SNR } & \text { signal-to-noise ratio } \\ \text { SPC } & \text { short packet communication } \\ \text { SSK } & \text { space shift keying } \\ \text { STLC } & \text { space-time line code } \\ \text { SWIPT } & \text { simultaneous wireless information } \\ & \text { and power transfer } \\ \text { TDMA } & \text { time division multiple access } \\ \text { THz } & \text { terahertz } \\ & \\ \text { UL } & \text { uplink } \\ \text { URLLC } & \text { ultra-reliable low latency communi- } \\ & \text { cation } \\ \text { V2V } & \text { vehicle-to-vehicle } \\ \text { VLC } & \text { visible light communication } \\ \text { WIT } & \text { wireless information transfer } \\ \text { WPT } & \text { wireless power transfer } \\ & \end{array}$

[1] W. Saad, M. Bennis, and M. Chen, "A vision of $6 \mathrm{G}$ wireless systems: Applications, trends, technologies, and open research problems," IEEE Net., vol. 34, no. 3, pp. 134-142, May/June 2020.

[2] S. Dang, O. Amin, B. Shihada, and M.-S. Alouini, "What should 6G be?" Nature Electronics, vol. 3, no. 1, pp. 20-29, 2020.

[3] "Ericsson mobility report, Q2 update, August 2021," Ericsson, Tech. Rep., 2021, an optional note. [Online]. Available: https: //www.ericsson.com/en/mobility-report.

[4] "Cisco annual Internet report (2018-2023)," Cisco, Tech Rep., 2020, an optional note. [Online]. Available: https: //www.cisco.com/c/en/us/solutions/collateral/executive-perspectives/ annual-internet-report/white-paper-c11-741490.pdf.

[5] M. Vaezi, R. Schober, Z. Ding, and H. V. Poor, "Non-orthogonal multiple access: Common myths and critical questions," IEEE Wireless Commun., vol. 26, no. 5, pp. 174-180, Oct. 2019.

[6] O. Maraqa, A. S. Rajasekaran, S. Al-Ahmadi, H. Yanikomeroglu, and S. M. Sait, "A survey of rate-optimal power domain NOMA with enabling technologies of future wireless networks," IEEE Commun. Surveys Tuts., vol. 22, no. 4, pp. 2192-2235, Fourth Quart. 2020.

[7] L. Dai, B. Wang, Z. Ding, Z. Wang, S. Chen, and L. Hanzo, "A survey of non-orthogonal multiple access for 5G," IEEE Commun. Surveys Tuts., vol. 20, no. 3, pp. 2294-2323, Third Quart. 2018.

[8] Y. Liu, Z. Qin, M. Elkashlan, Z. Ding, A. Nallanathan, and L. Hanzo, "Nonorthogonal multiple access for 5G and beyond," Proc. IEEE, vol 105, no. 12, pp. 2347-2381, Dec. 2017.

[9] Z. Xiang, W. Yang, Y. Cai, Z. Ding, Y. Song, and Y. Zou, "NOMAassisted secure short-packet communications in IoT," IEEE Wireless Commun., vol. 27, no. 4, pp. 8-15, Aug. 2020.

[10] A. I. Perez-Neira, M. Caus, and M. A. Vazquez, "Non-orthogonal transmission techniques for multibeam satellite systems," IEEE Commun. Mag., vol. 57, no. 12, pp. 58-63, Dec. 2019.

[11] Y. Liu, Z. Qin, Y. Cai, Y. Gao, G. Y. Li, and A. Nallanathan, "UAV communications based on non-orthogonal multiple access," IEEE Wireless Commun., vol. 26, no. 1, pp. 52-57, Feb. 2019.

[12] H. Marshoud, P. C. Sofotasios, S. Muhaidat, G. K. Karagiannidis, and B. S. Sharif, "On the performance of visible light communication systems with non-orthogonal multiple access," IEEE Trans. Wireless Commun., vol. 16, no. 10, pp. 6350-6364, Oct. 2017.

[13] M. Jain, N. Sharma, A. Gupta, D. Rawal, and P. Garg, "Performance analysis of NOMA assisted underwater visible light communication system," IEEE Wireless Commun. Lett., vol. 9, no. 8, pp. 1291-1294, Aug. 2020 
[14] S. M. R. Islam, N. Avazov, O. A. Dobre, and K.-s. Kwak, "Powerdomain non-orthogonal multiple access (NOMA) in 5G systems: Potentials and challenges," IEEE Commun. Surveys Tuts., vol. 19, no. 2 , pp. 721-742, Second Quart. 2017.

[15] Z. Ding, X. Lei, G. K. Karagiannidis, R. Schober, J. Yuan, and V. K. Bhargava, "A survey on non-orthogonal multiple access for 5G networks: Research challenges and future trends," IEEE J. Sel. Areas Commun., vol. 35, no. 10, pp. 2181-2195, Oct. 2017.

[16] Y. Wang, B. Ren, S. Sun, S. Kang, and X. Yue, "Analysis of nonorthogonal multiple access for 5G," China Commun., vol. 13, no. Supplement2, pp. 52-66, 2016.

[17] M. Basharat, W. Ejaz, M. Naeem, A. M. Khattak, and A. Anpalagan, "A survey and taxonomy on nonorthogonal multiple-access schemes for 5G networks," Trans. Emerg. Telecommun. Technol., vol. 29, no. 1, p. e3202, 2018. [Online]. Available: https://onlinelibrary.wiley.com/ doi/abs/10.1002/ett.3202

[18] Y. Cai, Z. Qin, F. Cui, G. Y. Li, and J. A. McCann, "Modulation and multiple access for 5G networks," IEEE Commun. Surveys Tuts. vol. 20, no. 1, pp. 629-646, First Quart. 2018.

[19] N. Ye, H. Han, L. Zhao, and A.-h. Wang, "Uplink nonorthogonal multiple access technologies toward 5G: A survey," Wireless Commun. Mobile Comput., vol. 2018, 2018. [Online]. Available: https://doi.org/10.1155/2018/6187580

[20] M. B. Shahab, R. Abbas, M. Shirvanimoghaddam, and S. J. Johnson, "Grant-free non-orthogonal multiple access for IoT: A survey," IEEE Commun. Surveys Tuts., vol. 22, no. 3, pp. 1805-1838, Third Quart. 2020.

[21] B. Makki, K. Chitti, A. Behravan, and M.-S. Alouini, "A survey of NOMA: Current status and open research challenges," IEEE Open J. Commun. Soc., vol. 1, pp. 179-189, Jan. 2020.

[22] H. Yahya, E. Alsusa, and A. Al-Dweik, "Exact BER analysis of NOMA with arbitrary number of users and modulation orders," IEEE Trans. Commun., vol. 69, no. 9, pp. 6330-6344, Sep. 2021.

[23] T. Assaf, A. Al-Dweik, M. S. E. Moursi, H. Zeineldin, and M. AlJarrah, "NOMA receiver design for delay-sensitive systems," IEEE Syst. J., pp. 1-12, 2020.

[24] C. Pan, H. Ren, K. Wang, J. F. Kolb, M. Elkashlan, M. Chen, M. Di Renzo, Y. Hao, J. Wang, A. L. Swindlehurst, X. You, and L. Hanzo, "Reconfigurable intelligent surfaces for 6G systems: Principles, applications, and research directions," IEEE Commun. Mag., vol. 59, no. 6, pp. 14-20, Jun. 2021.

[25] F. Jameel, S. Zeb, W. U. Khan, S. A. Hassan, Z. Chang, and J. Liu, "NOMA-enabled backscatter communications: Toward battery-free IoT networks," IEEE Internet Things Mag., vol. 3, no. 4, pp. 95-101, Dec. 2020.

[26] Z. Ding and H. Vincent Poor, "A simple design of irs-noma transmission," IEEE Commun. Lett., vol. 24, no. 5, pp. 1119-1123, May 2020.

[27] H. Marshoud, S. Muhaidat, P. C. Sofotasios, S. Hussain, M. A. Imran, and B. S. Sharif, "Optical non-orthogonal multiple access for visible light communication," IEEE Wireless Commun., vol. 25, no. 2, pp. 82-88, Apr. 2018.

[28] M. Le-Tran, T.-H. Vu, and S. Kim, "Performance analysis of optical backhauled cooperative NOMA visible light communication," IEEE Trans. Veh. Technol., pp. 1-1, Oct. 2021.

[29] M. A. Khalighi and M. Uysal, "Survey on free space optical communication: A communication theory perspective," IEEE Commun. Surveys Tuts., vol. 16, no. 4, pp. 2231-2258, Fourth Quart. 2014.

[30] M. Zeng, W. Hao, O. A. Dobre, and Z. Ding, "Cooperative NOMA State of the art, key techniques, and open challenges," IEEE Netw., vol. 34, no. 5, pp. 205-211, Sep./Oct. 2020.

[31] F. Kara and H. Kaya, "BER performances of downlink and uplink NOMA in the presence of SIC errors over fading channels," IET Commun., vol. 12, pp. 1834-1844(10), Sep. 2018.

[32] I. Lee and J. Kim, "Average symbol error rate analysis for nonorthogonal multiple access with M-ary QAM signals in Rayleigh fading channels," IEEE Commun. Lett., vol. 23, no. 8, pp. 1328-1331, Aug. 2019.

[33] E. C. Cejudo, H. Zhu, and O. Alluhaibi, "On the power allocation and constellation selection in downlink NOMA," in Proc. IEEE Veh. Technol. Conf. (VTC), Toronto, ON, Canada, Sep. 2017, pp. 1-5.

[34] Q. He, Y. Hu, and A. Schmeink, "Closed-form symbol error rate expressions for non-orthogonal multiple access systems," IEEE Trans. Veh. Technol., vol. 68, no. 7, pp. 6775-6789, July 2019.

[35] Y. Wang, J. Wang, L. Ma, Y. Huang, and C. Zhao, "Minimum error performance of downlink non-orthogonal multiple access systems," in
IEEE Veh. Technol. Conf. (VTC), Honolulu, HI, USA, Sep. 2019, pp. $1-5$.

[36] L. Bariah, S. Muhaidat, and A. Al-Dweik, "Error probability analysis of non-orthogonal multiple access over Nakagami- $m$ fading channels," IEEE Trans. Commun., vol. 67, no. 2, pp. 1586-1599, Feb. 2019.

[37] L. Bariah, B. Selim, L. Mohjazi, S. Muhaidat, P. C. Sofotasios, and W. Hamouda, "Pairwise error probability of non-orthogonal multiple access with I/Q imbalance," in UK/ China Emerging Technol. (UCET), Glasgow, United Kingdom, Aug. 2019, pp. 1-4.

[38] L. Bariah, S. Muhaidat, P. C. Sofotasios, S. Gurugopinath, W. Hamouda, and H. Yanikomeroglu, "Non-orthogonal multiple access in the presence of additive generalized Gaussian noise," IEEE Commun. Lett., vol. 24, no. 10, pp. 2137-2141, Oct. 2020.

[39] A. Almohamad, S. Althunibat, M. Hasna, and K. Qaraqe, "On the error performance of non-orthogonal multiple access systems," in Int. Conf. Inf. Commun. Technol. Convergence (ICTC), Jeju Island, Korea, Oct. 2020, pp. 116-121.

[40] K. Lee, J. S. Yeom, B. C. Jung, and J. Joung, "A novel non-orthogonal multiple access with space-time line codes for massive IoT networks," in IEEE Veh. Technol. Conf. (VTC), Honolulu, HI, USA, Sep. 2019, pp. 1-5.

[41] Y. Iraqi and A. Al-Dweik, "Power allocation for reliable SIC detection of rectangular QAM-based NOMA systems," IEEE Trans. Veh. Technol., vol. 70, no. 8, pp. 8355-8360, Aug. 2021.

[42] F. Kara and H. Kaya, "A true power allocation constraint for nonorthogonal multiple access with M-QAM signalling," in IEEE Microw. Theory Techn. Wireless Commun. (MTTW), vol. 1, Riga, Latvia, Oct. 2020, pp. 7-12.

[43] A. Hilario-Tacuri, "Closed-form expressions for symbol error rate of two-user NOMA systems," in IEEE Int. Conf. Electron., Elect. Eng. Comput. (INTERCON), Lima, Peru, Sep. 2021, pp. 1-4.

[44] T. Assaf, A. J. Al-Dweik, M. S. E. Moursi, H. Zeineldin, and M. AlJarrah, "Exact bit error-rate analysis of two-user NOMA using QAM with arbitrary modulation orders," IEEE Commun. Lett., vol. 24, no. 12, pp. 2705-2709, Dec. 2020.

[45] M. Jain, S. Soni, N. Sharma, and D. Rawal, "Performance analysis at far and near user in NOMA based system in presence of SIC error," Int. J. Electron. Commun. (AEU), vol. 114, p. 152993, Feb. 2020.

[46] A. Hilario-Tacuri, J. Maldonado, M. Revollo, and H. Chambi, "Bit error rate analysis of NOMA-OFDM in 5G systems with non-linear HPA with memory," IEEE Access, vol. 9, pp. 83709-83 717, Jun. 2021.

[47] K. Chung, "Impact of channel estimation errors on SIC performance of NOMA in 5G systems," J. Convergence Inf. Technol., vol. 10, no. 9, pp. 22-27, Sep. 2020. [Online]. Available: https://doi.org/10. 22156/CS4SMB.2020.10.09.022

[48] —, "Optimal detection for NOMA systems with correlated information sources of interactive mobile users," J. Korea Inst. Electron. Commun. Sci., vol. 15, no. 4, pp. 651-658, Aug. 2020. [Online]. Available: http://dx.doi.org/10.13067/JKIECS.2020.15.4.651

[49] _ "On negative correlation bit-to-symbol(: B2S) mapping for NOMA with correlated information sources in $5 \mathrm{G}$ systems," J. Korea Inst. Electron. Commun. Sci., vol. 15, no. 5, pp. 881-888, Oct. 2020. [Online]. Available: http://dx.doi.org/10.13067/JKIECS.2020.15.5.881

[50] A. Abushattal, S. Althunibat, M. Qaraqe, and H. Arslan, "A secure downlink NOMA scheme against unknown internal eavesdroppers," IEEE Wireless Commun. Lett., vol. 10, no. 6, pp. 1281-1285, Jun. 2021.

[51] A. M. A. Alkhazzar and H. Aghaeinia, "Symbol error rate analysis of non-orthogonal multiple access systems," Phys. Commun., vol. 46, p. 101295, Feb. 2021. [Online]. Available: https://www.sciencedirect. com/science/article/pii/S187449072100032X

[52] T. Assaf, A. Al-Dweik, M. E. Moursi, and H. Zeineldin, "Exact BER performance analysis for downlink NOMA systems over Nakagami- $m$ fading channels," IEEE Access, vol. 7, pp. 134 539-134 555, Sep. 2019.

[53] J.-R. Garnier, A. Fabre, H. Farès, and R. Bonnefoi, "On the performance of QPSK modulation over downlink NOMA: From error probability derivation to SDR-based validation," IEEE Access, vol. 8, pp. 66495-66507, Mar. 2020.

[54] M. Aldababsa, C. Göztepe, G. K. Kurt, and O. Kucur, "Bit error rate for NOMA network," IEEE Commun. Lett., vol. 24, no. 6, pp. 1188-1191, June 2020

[55] N. Jaiswal and N. Purohit, "Performance of downlink NOMA-enabled vehicular communications over double Rayleigh fading channels," IET Commun., vol. 14, no. 20, pp. 3652-3660, Sep. 2020.

[56] S. Mukhtar and G. R. Begh, "On the performance analysis of downlink NOMA system over non-homogenous fading channel," in IEEE Int. 
Conf. Comput. Power Commun. Technol. (GUCON), Kuala Lumpur, Malaysia, Nov. 2021, pp. 1-6.

[57] A. S. Alqahtani and E. Alsusa, "Performance analysis of downlink NOMA system over $\alpha-\eta-\mu$ generalized fading channel," in IEEE Veh. Technol. Conf. (VTC), Antwerp, Belgium, May 2020, pp. 1-5.

[58] B. M. ElHalawany, F. Jameel, D. B. da Costa, U. S. Dias, and $\mathrm{K}$. Wu, "Performance analysis of downlink NOMA systems over $\kappa-\mu$ shadowed fading channels," IEEE Trans Veh. Technol., vol. 69, no. 1, pp. 1046-1050, Jan. 2020 .

[59] X. Yan, H. Xiao, C. Wang, K. An, A. T. Chronopoulos, and G. Zheng, "Performance analysis of NOMA-based land mobile satellite networks," IEEE Access, vol. 6, pp. 31 327-31 339, June 2018.

[60] A. Qahtani, E. A. Alsusa, A. J. Al-Dweik, and M. Al-Jarrah, "Performance analysis for downlink NOMA over $\alpha-\mu$ generalized fading channels," IEEE Trans. Veh. Technol., pp. 1-1, 2021.

[61] K. Aslan and T. Gucluoglu, "Performance analysis of downlinkNOMA over generalized-k fading channels," Phys. Commun., vol. 48, p. 101414, Jul. 2021. [Online]. Available: https://www.sciencedirect. com/science/article/pii/S1874490721001518

[62] X. Wang, F. Labeau, and L. Mei, "Closed-form BER expressions of QPSK constellation for uplink non-orthogonal multiple access," IEEE Commun. Lett., vol. 21, no. 10, pp. 2242-2245, Oct. 2017.

[63] X. Chen, H. Yin, and G. Wei, "Impact of frequency offset on the performance of uplink OFDM-NOMA systems," in IEEE Int. Conf. Comput. Commun. (ICCC), Chengdu, China, Dec. 2018, pp. 168-173.

[64] F. Wei, T. Zhou, T. Xu, and H. Hu, "BER analysis for uplink NOMA in asymmetric channels," IEEE Commun. Lett., vol. 24, no. 11, pp. 2435-2439, Nov. 2020.

[65] R. P. Sirigina, A. S. Madhukumar, and M. Bowyer, "BER analysis of interference-limited synchronous wireless networks with line-of-sight links," IEEE Commun. Lett., vol. 23, no. 3, pp. 542-545, Mar. 2019.

[66] J. S. Yeom, H. S. Jang, K. S. Ko, and B. C. Jung, "BER performance of uplink NOMA with joint maximum-likelihood detector," IEEE Trans. Veh. Technol., vol. 68, no. 10, pp. 10295-10300, Oct. 2019.

[67] C. Liu and N. C. Beaulieu, "Exact SER and BER analysis on jointly optimal maximum likelihood detection of two QPSK signals with phase offset," IEEE Trans. Veh. Technol., vol. 70, no. 11, pp. 11695-11 709, Nov. 2021

[68] H. Semira, F. Kara, H. Kaya, and H. Yanikomeroglu, "Multi-user joint maximum-likelihood detection in uplink NOMA-IoT networks: Removing the error floor," IEEE Wireless Commun. Lett., vol. 10, no. 11, pp. 2459-2463, Nov. 2021.

[69] E. Carmona Cejudo, H. Zhu, and J. Wang, "Resource allocation in multicarrier NOMA systems based on optimal channel gain ratios," IEEE Trans. Wireless Commun., pp. 1-1, 2021.

[70] T. Assaf, A. J. Al-Dweik, M. Elmoursi, and H. Zeineldin, "Efficient bit loading algorithm for OFDM-NOMA systems with BER constraints," IEEE Trans. Veh. Technol., pp. 1-1, Nov. 2021.

[71] W. Yu, H. Jia, and L. Musavian, "Joint adaptive M-QAM modulation and power adaptation for a downlink NOMA network," IEEE Trans. Commun., pp. 1-1, Nov. 2021.

[72] K. Wang, T. Zhou, T. Xu, H. Hu, and X. Tao, "Asymmetric adaptive modulation for uplink NOMA systems," IEEE Trans. Commun., vol. 69, no. 11, pp. 7222-7235, Nov. 2021.

[73] Y. Wang, J. Wang, D. W. K. Ng, R. Schober, and X. Gao, "A minimum error probability NOMA design," IEEE Trans. Wireless Commun., pp. $1-1,2021$.

[74] A. K. Dutta, "MBER criterion assisted power NOMA design and performance analysis with estimated channel," IEEE Trans. Veh. Technol., vol. 68 , no. 12 , pp. $11816-11826$, Dec. 2019.

[75] Z. Dong, H. Chen, J. K. Zhang, L. Huang, and B. Vucetic, "Uplink nonorthogonal multiple access with finite-alphabet inputs," IEEE Trans. Wireless Commun., vol. 17, no. 9, pp. 5743-5758, Sep. 2018.

[76] C. Wang, Y. Wang, and P. Xiao, "Power allocation based on SINR balancing for NOMA systems with imperfect channel estimation," in Int. Conf. Signal Process. Commun. Syst. (ICSPCS), Gold Coast, Australia, Dec. 2019, pp. 1-6.

[77] S. Shieh, C. Lin, Y. Huang, and C. Wang, "On Gray labeling for downlink non-orthogonal multiple access without SIC," IEEE Commun. Lett., vol. 20, no. 9, pp. 1721-1724, Sep. 2016.

[78] W. Han, Y. Wang, D. Tang, R. Hou, and X. Ma, "Study of the symbol error rate/bit error rate in non-orthogonal multiple access (NOMA) systems," in IEEE/CIC Int. Conf. Commun. in China (ICCC Workshops), Beijing, China, Aug. 2018, pp. 101-106.

[79] W. Han, X. Ma, D. Tang, and N. Zhao, "Study of SER and BER in NOMA systems," IEEE Trans. Veh. Technol., vol. 70, no. 4, pp. 33253340, May 2021.
[80] J. Yeom, E. Chu, B. Jung, and H. Jin, "Performance analysis of diversity-controlled multi-user superposition transmission for 5G wireless networks," Sensors, vol. 18, no. 2, p. 536, Feb. 2018. [Online]. Available: http://www.mdpi.com/1424-8220/18/2/536

[81] Q. Gao, M. Jia, Q. Guo, and X. Gu, "On the analysis and optimization of BER performance of symmetrical coding based NOMA," in IEEE Wireless Commun. Netw. Conf. Workshops (WCNCW), May 2021, pp. $1-6$.

[82] J. Zhang, X. Wang, T. Hasegawa, and T. Kubo, "Downlink nonorthogonal multiple access (NOMA) constellation rotation," in IEEE Veh. Technol. Conf. (VTC), Montreal, QC, Canada, Sep. 2016, pp. 1-5.

[83] Y. Chang and K. Fukawa, "Non-orthogonal multiple access with phase rotation employing joint mud and sic," in IEEE Veh. Technol. Conf. (VTC), Porto, Portugal, June 2018, pp. 1-5.

[84] — "Phase rotated non-orthogonal multiple access for 3-user superposition signals," in IEEE Veh. Technol. Conf. (VTC), Honolulu, HI, USA, Sep. 2019, pp. 1-5.

[85] B. K. Ng and C. T. Lam, "On joint modulation design in two-user nonorthogonal multiple access channels," in Int. Symp. Wireless Commun. Syst.s (ISWCS), Poznan, Poland, Oct. 2016, pp. 65-69.

[86] B. K. Ng and C.-T. Lam, "Joint power and modulation optimization in two-user non-orthogonal multiple access channels: A minimum error probability approach," IEEE Trans. Veh. Technol., vol. 67, no. 11, pp. 10 693-10 703, Nov. 2018.

[87] X. Guan, Q. Yang, Y. Hong, and C. C.-K. Chan, "Nonorthogonal multiple access with phase pre-distortion in visible light communication," Opt. Express, vol. 24, no. 22, pp. $25816-$ 25823 , Oct. 2016. [Online]. Available: http://www.osapublishing.org/ oe/abstract.cfm?URI=oe-24-22-25816

[88] M. Qiu, Y.-C. Huang, and J. Yuan, "Downlink NOMA without SIC for fast fading channels: Lattice partitions with algebraic rotations," in IEEE Int. Conf. Commun. (ICC), Shanghai, China, July 2019, pp. 1-6.

[89] M. Qiu, Y. C. Huang, and J. Yuan, "Downlink non-orthogonal multiple access without SIC for block fading channels: An algebraic rotation approach," IEEE Trans. Wireless Commun., vol. 18, no. 8, pp. 39033918, Aug. 2019.

[90] N. Ye, A. Wang, X. Li, W. Liu, X. Hou, and H. Yu, "On constellation rotation of NOMA with SIC receiver," IEEE Commun. Lett., vol. 22, no. 3, pp. 514-517, Mar. 2018.

[91] S. Ozyurt and O. Kucur, "Quadrature NOMA: A low-complexity multiple access technique with coordinate interleaving," IEEE Wireless Commun. Lett., vol. 9, no. 9, pp. 1452-1456, Sep. 2020.

[92] A. Chauhan and A. Jaiswal, "Non-orthogonal multiple access: A constellation domain multiplexing approach," in IEEE Int. Symp. Personal Indoor Mobile Radio Commun. (PIMRC), London, United Kingdom, Aug./Sep. 2020, pp. 1-6.

[93] C. H. Lin, S. L. Shieh, T. C. Chi, and P. N. Chen, "Optimal interconstellation rotation based on minimum distance criterion for uplink NOMA," IEEE Trans. Veh. Technol., vol. 68, no. 1, pp. 525-539, Jan. 2019.

[94] H.-P. Liu, S.-L. Shieh, C.-H. Lin, and P.-N. Chen, "A minimum distance criterion based constellation design for uplink NOMA," in IEEE Veh Technol. Conf. (VTC), Honolulu, HI, USA, Nov. 2019, pp. 1-5.

[95] H.-P. Liu, Y.-C. Huang, S.-L. Shieh, and P.-N. Chen, "Power-domain downlink NOMA constellation design with heterogeneous reliability requirements," in Int. Conf. Signal Processing Commun. Syst. (ICSPCS), Adelaide, SA, Australia, Feb. 2021, pp. 1-6.

[96] Y. Zhao, J. Hu, Z. Ding, and K. Yang, "Constellation rotation aided modulation design for the multi-user SWIPT-NOMA," in IEEE Int. Commun. Conf. (ICC), Kansas City, MO, USA, May 2018.

[97] _ "Joint interleaver and modulation design for multi-user SWIPTNOMA," IEEE Trans. Commun., vol. 67, no. 10, pp. 7288-7301, Oct. 2019.

[98] X. Wan, X. Zhu, Y. Jiang, Y. Liu, and J. Zhao, "An interference alignment and ICA-based semiblind dual-user downlink NOMA system for high-reliability low-latency IoT," IEEE Internet Things $J$, vol. 7 , no. 11, pp. 10837-10851, Apr. 2020.

[99] H. Yahya, A. Al-Dweik, and E. Alsusa, "Power-tolerant NOMA using data-aware adaptive power assignment for IoT systems," IEEE Internet Things J., vol. 8, no. 19, pp. 14 896-14907, Oct. 2021.

[100] H. Yahya, E. Alsusa, and A. Al-Dweik, "Enhanced non-orthogonal multiple access using data-aware power assignment," Int. Symp. Netw. Comput. Commun. (ISNCC), pp. 1-6, Nov. 2021.

[101] P. Li, Z. Ding, and K. Feng, "Enhanced receiver based on FEC code constraints for uplink NOMA with imperfect CSI," IEEE Trans. Wireless Commun., vol. 18, no. 10, pp. 4790-4802, Oct. 2019. 
[102] X. Zou, M. Ganji, and H. Jafarkhani, "Trellis-coded non-orthogonal multiple access," IEEE Wireless Commun. Lett., vol. 9, no. 4, pp. 538542, Apr. 2020.

[103] B. K. Ng and C.-T. Lam, "A new two-user binary polar coded multiple access scheme with power and rate allocation," IEEE Wireless Commun. Lett., vol. 10, no. 10, pp. 2299-2303, Oct. 2021

[104] C. Yan, A. Harada, A. Benjebbour, Y. Lan, A. Li, and H. Jiang, "Receiver design for downlink non-orthogonal multiple access (NOMA)," in IEEE Veh. Technol. Conf. (VTC), July 2015.

[105] K. Xiao, B. Xia, L. Ma, and Y. Wei, "Design and analysis of probabilistic shaping scheme for uplink nonorthogonal multiple access systems," IEEE Trans. Commun., vol. 67, no. 12, pp. 8818-8833, Dec. 2019.

[106] S. Baig, U. Ali, H. M. Asif, A. A. Khan, and S. Mumtaz, "Closed-form BER expression for Fourier and wavelet transform-based pulse-shaped data in downlink NOMA," IEEE Commun. Lett., vol. 23, no. 4, pp. 592-595, Apr. 2019

[107] A. Maatouk, E. Caliskan, M. Koca, M. Assaad, G. Gui, and H. Sari, "Frequency-domain NOMA with two sets of orthogonal signal waveforms," IEEE Commun. Lett., vol. 22, no. 5, pp. 906-909, May. 2018.

[108] A. A. Khansa, X. Chen, Y. Yin, G. Gui, and H. Sari, "Performance analysis of power-domain NOMA and NOMA-2000 on AWGN and Rayleigh fading channels," Phys. Commun., vol. 43, p. 101185, Dec. 2020

[109] X. Zhang, Z. Wang, X. Ning, and H. Xie, "On the performance of GFDM assisted NOMA schemes," IEEE Access, vol. 8, pp. $88961-$ 88968, May 2020.

[110] H. Haci, H. Zhu, and J. Wang, "A novel interference cancellation technique for non-orthogonal multiple access (NOMA)," in IEEE Global Commun. Conf. (GLOBECOM), San Diego, CA, USA, Feb. 2016, pp. 1-6.

[111] H. Haci, H. Zhu, and J. Wang, "Performance of non-orthogonal multiple access with a novel asynchronous interference cancellation technique," IEEE Trans. Commun., vol. 65, no. 3, pp. 1319-1335, Mar. 2017

[112] C. Liu and N. C. Beaulieu, "Exact BER performance for symbolasynchronous two-user non-orthogonal multiple access," IEEE Commun. Lett., vol. 25, no. 3, pp. 764-768, Mar. 2021.

[113] H. S. Ghazi and K. W. Wesołowski, "Improved detection in successive interference cancellation NOMA OFDM receiver," IEEE Access, vol. 7, pp. 103 325-103 335, July 2019

[114] H. Xu and N. Pillay, "Threshold based signal detection and the average symbol error probability for downlink NOMA systems with M-ary QAM," IEEE Access, vol. 8, pp. 156677-156685, Aug. 2020.

[115] A. Al-Dweik, Y. Iraqi, K.-H. Park, M. Al-Jarrah, E. Alsusa, and M.-S Alouini, "Efficient NOMA design without channel phase information using amplitude-coherent detection," IEEE Trans. Commun., pp. 1-1, 2021.

[116] H. Huang, J. Wang, J. Wang, J. Yang, J. Xiong, and G. Gui, "Symbol error rate performance analysis of non-orthogonal multiple access for visible light communications," China Commun., vol. 14, no. 12, pp. 153-161, Dec. 2017

[117] H. Marshoud, P. C. Sofotasios, S. Muhaidat, G. K. Karagiannidis, and B. S. Sharif, "Error performance of NOMA VLC systems," in IEEE Int. Conf. Commun. (ICC), Paris, France, May 2017, pp. 1-6.

[118] X. Liu, Y. Wang, F. Zhou, and R. Q. Hu, "BER analysis for NOMAenabled visible light communication systems with M-PSK," in Int. Conf. Wireless Commun. Signal Process. (WCSP), Hangzhou, China, Oct. 2018, pp. 1-7.

[119] X. Liu, Z. Chen, Y. Wang, F. Zhou, Y. Luo, and R. Q. Hu, "BER analysis of NOMA-enabled visible light communication systems with different modulations," IEEE Trans. Veh. Technol., vol. 68, no. 11, pp. 10 807-10 821, Nov. 2019.

[120] E. M. Almohimmah and M. T. Alresheedi, "Error analysis of NOMAbased VLC systems with higher order modulation schemes," IEEE Access, vol. 8, pp. 2792-2803, Dec. 2020.

[121] T. Cao, H. Zhang, and J. Song, "BER performance analysis for downlink nonorthogonal multiple access with error propagation mitigated method in visible light communications," IEEE Trans. Veh. Technol., vol. 70, no. 9, pp. 9190-9206, Sep. 2021.

[122] J. Song, T. Cao, and H. Zhang, "Performance analysis of a lowcomplexity nonorthogonal multiple access scheme in visible light communication downlinks using pulse modulations," Intell. Converged Netw., vol. 2, no. 1, pp. 50-65, Mar. 2021.

[123] H. Ren, Z. Wang, S. Du, Y. He, J. Chen, S. Han, C. Yu, C. $\mathrm{Xu}$, and J. Yu, "Performance improvement of NOMA visible light communication system by adjusting superposition constellation: a convex optimization approach," Opt. Express, vol. 26, no. 23, pp. 29796-29806, Nov. 2018. [Online]. Available: http://www. osapublishing.org/oe/abstract.cfm?URI=oe-26-23-29796

[124] V. Dixit and A. Kumar, "An exact error analysis of multi-user RC/MRC based MIMO-NOMA-VLC system with imperfect SIC," IEEE Access, vol. 9, pp. 136710-136720, Oct. 2021.

[125] — "An exact BER analysis of NOMA-VLC system with imperfect SIC and CSI," Int. J. Electron. Commun., vol. 138, pp. 1434-8411, Aug. 2021. [Online]. Available: https://doi.org/10.1016/j.aeue.2021. 153864

[126] — "BER analysis of dynamic FOV based MIMO-NOMA-VLC system," Int. J. Electron. Commun., vol. 142, p. 153989, Nov. 2021. [Online]. Available: https://www.sciencedirect.com/science/article/pii/ S1434841121003861

[127] R. Mitra and V. Bhatia, "Precoded Chebyshev-NLMS-based predistorter for nonlinear LED compensation in NOMA-VLC," IEEE Trans. Commun., vol. 65, no. 11, pp. 4845-4856, Nov. 2017.

[128] P. Saxena and M. R. Bhatnagar, "1-bit feedback-based beamforming scheme for an uplink FSO-NOMA system with SIC errors," Appl. Opt., vol. 59, no. 36, pp. 11274-11291, Dec. 2020. [Online]. Available: http://ao.osa.org/abstract.cfm?URI=ao-59-36-11274

[129] V. C. Thirumavalavan and T. S. Jayaraman, "BER analysis of reconfigurable intelligent surface assisted downlink power domain NOMA system," in Int. Conf. Commun. Syst. Netw. (COMSNETS), Bengaluru, India, Mar. 2020, pp. 519-522.

[130] L. Bariah, S. Muhaidat, P. C. Sofotasios, F. E. Bouanani, O. A. Dobre, and W. Hamouda, "Large intelligent surface-assisted nonorthogonal multiple access for 6G networks: Performance analysis," IEEE Internet Things J., vol. 8, no. 7, pp. 5129-5140, Apr. 2021

[131] A. W. Nazar, S. A. Hassan, H. Jung, A. Mahmood, and M. Gidlund, "BER analysis of a backscatter communication system with nonorthogonal multiple access," IEEE Trans. Green Commun. Netw., vol. 5 , no. 2, pp. 574-586, Jun. 2021.

[132] S. Rajkumar and D. N. K. Jayakody, "Backscatter assisted NOMAPLNC based wireless networks," Sensors, vol. 21, no. 22, Nov. 2021. [Online]. Available: https://www.mdpi.com/1424-8220/21/22/7589

[133] S. Li, L. Bariah, S. Muhaidat, P. Sofotasios, J. Liang, and A. Wang, "Error analysis of NOMA-based user cooperation with SWIPT," in Int. Conf. Distrib. Comput. Sensor Syst. (DCOSS), Santorini Island, Greece, Greece, May 2019, pp. 507-513.

[134] L. Bariah, S. Muhaidat, and A. Al-Dweik, "Error performance of NOMA-based cognitive radio networks with partial relay selection and interference power constraints," IEEE Trans. Commun., vol. 68, no. 2, pp. 765-777, Feb. 2020.

[135] F. Kara and H. Kaya, "Error probability analysis of NOMA-based diamond relaying network," IEEE Trans. Veh. Technol., vol. 69, no. 2 , pp. 2280-2285, Feb. 2020.

[136] _ "Improved error performance in NOMA-based diamond relaying," in IEEE Microw. Theory Techn. Wireless Commun. (MTTW), vol. 1, Riga, Latvia, Oct. 2020, pp. 151-156.

[137] _ , "Threshold-based selective cooperative-NOMA," IEEE Commun Lett., vol. 23, no. 7, pp. 1263-1266, July 2019

[138] _ "On the error performance of cooperative-NOMA with statistical CSIT," IEEE Commun. Lett., vol. 23, no. 1, pp. 128-131, Jan. 2019.

[139] L. Mohjazi, L. Bariah, S. Muhaidat, P. C. Sofotasios, O. Onireti, an M. A. Imran, "Error probability analysis of non-orthogonal multiple access for relaying networks with residual hardware impairments," in IEEE Int. Symp. Personal Indoor Mobile Radio Commun. (PIMRC), Istanbul, Turkey, Sep. 2019, pp. 1-6.

[140] M. Jain, N. Sharma, A. Gupta, D. Rawal, and P. Garg, "Performance analysis of NOMA assisted mobile Ad hoc networks for sustainable future radio access," IEEE Trans. Sustain. Comput., pp. 1-1, 2020.

[141] D. Zhu, Y. Yao, L. Xu, H. Zhang, H. Fang, and X. Li, "Analysis of asymptotically tight approximation SER for cooperative NOMA systems," in Proc. IEEE Int. Conf. Commun. Technol. (ICCT), Xi' an, China, Oct. 2019, pp. 34-39.

[142] F. Kara and H. Kaya, "Improved user fairness in decode-forward relaying non-orthogonal multiple access schemes with imperfect SIC and CSI," IEEE Access, vol. 8, pp. 97 540-97 556, May 2020.

[143] M. Li, S. Huang, L. Tian, O. Alhussein, and S. Muhaidat, "Error rate performance of NOMA system with full-duplex cooperative relaying," Phys. Commun., vol. 49, p. 101447, Dec. 2021.

[144] F. Kara, H. Kaya, and H. Yanikomeroglu, "A lightweight machine learning assisted power optimization for minimum error in NOMACRS over Nakagami-m channels," IEEE Trans. Veh. Technol., vol. 70 , no. 10 , pp. $11067-11072$, Oct. 2021 
[145] M. Shen, Z. Huang, X. Lei, and L. Fan, "BER analysis of NOMA with max-min relay selection," China Commun., vol. 18, no. 7, pp. 172-182, Jul. 2021.

[146] L. Bariah, S. Muhaidat, and A. Al-Dweik, "Error probability analysis of NOMA-based relay networks with SWIPT," IEEE Commun. Lett., vol. 23, no. 7, pp. 1223-1226, July 2019 .

[147] S. Li, A. Wang, and J. Liang, "PEP analysis of AF relay NOMA systems employing order statistics of cascaded channels," Electron., vol. 8, no. 6, June 2019. [Online]. Available: https: //www.mdpi.com/2079-9292/8/6/695

[148] S. Li, L. Bariah, S. Muhaidat, P. C. Sofotasios, J. Liang, and A. Wang, "SWIPT-enabled cooperative NOMA with $m$ th best relay selection," IEEE Open J. Commun. Soc., vol. 1, pp. 1798-1807, Nov. 2020.

[149] F. Kara, "Error performance of cooperative relaying systems empowered by SWIPT and NOMA," Phys. Commun., vol. 49, p. 101450, Dec. 2021. [Online]. Available: https://www.sciencedirect. com/science/article/pii/S1874490721001877

[150] S. Üstünbaş and U. Aygölü, "BER analysis of non-orthogonal multiple access-based cognitive butterfly network," Electron. Lett., vol. 56, no. 7, pp. 357-360, Mar. 2020. [Online]. Available: https://onlinelibrary.wiley.com/doi/10.1049/el.2019.3618

[151] S. Dhanasekaran and T. R. Sushma, "Performance analysis of cellcenter users in NOMA based-spectrum sharing protocol," IEEE Commun. Lett., vol. 24, no. 1, pp. 52-56, Jan. 2020.

[152] K. Sultan, "Best relay selection schemes for NOMA based cognitive relay networks in underlay spectrum sharing," IEEE Access, vol. 8, pp. $190160-190172$, Oct. 2020.

[153] H. Sadat, M. Abaza, S. M. Gasser, and H. ElBadawy, "Performance analysis of cooperative non-orthogonal multiple access in visible light communication," Appl. Sci., vol. 9, no. 19, p. 4004, Sep. 2019. [Online]. Available: https://www.mdpi.com/2076-3417/9/19/4004

[154] P. Yang, Y. Xiao, M. Xiao, and Z. Ma, "NOMA-aided precoded spatial modulation for downlink MIMO transmissions," IEEE J. Sel. Topics Signal Process., vol. 13, no. 3, pp. 729-738, June 2019.

[155] F. Kara and H. Kaya, "Performance analysis of SSK-NOMA," IEEE Trans. Veh. Technol., vol. 68, no. 7, pp. 6231-6242, July 2019.

[156] —, "Spatial multiple access (SMA): Enhancing performances of MIMO-NOMA systems," in Int. Conf. Telecomm. Signal Process. (TSP), Budapest, Hungary, July 2019, pp. 466-471.

[157] J. Li, J. Hou, L. Fan, Y. Yan, X. Q. Jiang, and H. Hai, "NOMA-aided generalized pre-coded quadrature spatial modulation for downlink communication systems," China Commun., vol. 17, no. 11, pp. 120 130, Nov. 2020.

[158] N. Jaiswal and N. Purohit, "Performance analysis of NOMA-enabled vehicular communication systems with transmit antenna selection over double nakagami- $m$ fading," IEEE Trans. Veh. Technol., pp. 1-1, 2021.

[159] X. Zhu, Z. Wang, and J. Cao, "NOMA-based spatial modulation," IEEE Access, vol. 5, pp. 3790-3800, Mar. 2017.

[160] Q. Si, M. Jin, Y. Chen, N. Zhao, and X. Wang, "Performance analysis of spatial modulation aided NOMA with full-duplex relay," IEEE Trans. Veh. Technol., vol. 69, no. 5, pp. 5683-5687, May 2020.

[161] A. Shehni and M. F. Flanagan, "Distributed spatial modulation aided NOMA," in European Conf. Netw. Commun. (EuCNC), Valencia, Spain, June 2019, pp. 325-330.

[162] J. Li, S. Dang, Y. Yan, Y. Peng, S. Al-Rubaye, and A. Tsourdos, "Generalized quadrature spatial modulation and its application to vehicular networks with NOMA," IEEE Trans. Intell. Transp. Syst., pp. 1-10, July 2020

[163] Q. Li, M. Wen, E. Basar, H. V. Poor, and F. Chen, "Spatial modulationaided cooperative NOMA: Performance analysis and comparative study," IEEE J. Sel. Topics Signal Process., vol. 13, no. 3, pp. 715-728, June 2019.

[164] M. Can, I. Altunbas, and E. Basar, "NOMA-based downlink relaying with media-based modulation," Phys. Commun., vol. 41, p. 101116 , Aug. 2020

[165] X. Chen, M. Wen, T. Mao, and S. Dang, "Spectrum resource allocation based on cooperative NOMA with index modulation," IEEE Trans. Cogn. Commun. Netw., vol. 6, no. 3, pp. 946-958, Sep. 2020.

[166] X. Chen, M. Wen, and S. Dang, "On the performance of cooperative OFDM-NOMA system with index modulation," IEEE Wireless Commun. Lett., vol. 9, no. 9, pp. 1346-1350, Sep. 2020.

[167] A. Tusha, S. Doğan, and H. Arslan, "A hybrid downlink NOMA with OFDM and OFDM-IM for beyond 5G wireless networks," IEEE Signal Process. Lett., vol. 27, pp. 491-495, 2020.

[168] S. Doğan, A. Tusha, and H. Arslan, "NOMA with index modulation for uplink URLLC through grant-free access," IEEE J. Sel. Topics Signal Process., vol. 13, no. 6, pp. 1249-1257, Oct. 2019.
[169] A. Almohamad, S. Althunibat, M. Hasna, and K. Qaraqe, "A downlink index-modulation based nonorthogonal multiple access scheme," in IEEE Int. Symp. Personal Indoor Mobile Radio Commun. (PIMRC), London, United Kingdom, Aug./Sep. 2020, pp. 1-6.

[170] M. B. Shahab, S. J. Johnson, M. Shirvanimoghaddam, M. Chafii, E. Basar, and M. Dohler, "Index modulation aided uplink NOMA for massive machine type communications," IEEE Wireless Commun. Lett., vol. 9, no. 12, pp. 2159-2162, Dec. 2020.

[171] S. Althunibat, R. Mesleh, and T. F. Rahman, "A novel uplink multiple access technique based on index-modulation concept," IEEE Trans. Commun., vol. 67, no. 7, pp. 4848-4855, July 2019.

[172] K. Lai, J. Lei, L. Wen, and G. Chen, "Codeword position index modulation design for sparse code multiple access system," IEEE Trans. Veh. Technol., vol. 69, no. 11, pp. 13273-13288, Nov. 2020.

[173] X. Lai, Q. Zhang, and J. Qin, "Cooperative NOMA short-packet communications in flat Rayleigh fading channels," IEEE Trans. Veh. Technol., vol. 68, no. 6, pp. 6182-6186, June 2019.

[174] T.-H. Vu, T.-V. Nguyen, T.-T. Nguyen, and S. Kim, "Performance analysis and deep learning design of wireless powered cognitive NOMA IoT short-packet communications with imperfect CSI and SIC,' IEEE Internet Things J., pp. 1-1, 2021.

[175] J. Zheng, Q. Zhang, and J. Qin, "Average block error rate of downlink NOMA short-packet communication systems in Nakagami- $m$ fading channels," IEEE Commun. Lett., vol. 23, no. 10, pp. 1712-1716, Oct. 2019.

[176] Y. Yu, H. Chen, Y. Li, Z. Ding, and B. Vucetic, "On the performance of non-orthogonal multiple access in short-packet communications," IEEE Commun. Lett., vol. 22, no. 3, pp. 590-593, Mar. 2018.

[177] L. Yuan, Z. Zheng, N. Yang, and J. Zhang, "Performance analysis of short-packet non-orthogonal multiple access with Alamouti space-time block coding," IEEE Trans. Veh. Technol., vol. 70, no. 3, pp. 29002905, Mar. 2021.

[178] X. Lai, T. Wu, Q. Zhang, and J. Qin, "Average secure BLER analysis of NOMA downlink short-packet communication systems in flat Rayleigh fading channels," IEEE Trans. Wireless Commun., pp. 1-1, 2020.

[179] X. Lai, Q. Zhang, and J. Qin, "Downlink NOMA networks with hybrid long-packet and short-packet communications in flat rayleigh fading channels," IEEE Syst. J., vol. 14, no. 3, pp. 3410-3413, Sep. 2020.

[180] W. B. Ameur, P. Mary, M. Dumay, J. Hélard, and J. Schwoerer, "Power allocation for BER minimization in an uplink MUSA scenario," in IEEE Veh. Technol. Conf. (VTC), Antwerp, Belgium, May 2020, pp. $1-5$.

[181] J. Bao, Z. Ma, G. K. Karagiannidis, M. Xiao, and Z. Zhu, "Joint multiuser detection of multidimensional constellations over fading channels," in IEEE Trans. Commun., vol. 65, no. 1, Jan. 2017, pp. $161-172$

[182] Y. Liu, L. Yang, P. Xiao, H. Haas, and L. Hanzo, "Spatial modulated multicarrier sparse code-division multiple access," IEEE Trans. Wireless Commun., vol. 19, no. 1, pp. 610-623, Jan. 2020.

[183] L. Yu, P. Fan, X. Lei, and P. T. Mathiopoulos, "BER analysis of SCMA systems with codebooks based on star-QAM signaling constellations," IEEE Commun. Lett., vol. 21, no. 9, pp. 1925-1928, Sep. 2017.

[184] L. Yu, P. Fan, D. Cai, and Z. Ma, "Design and analysis of SCMA codebook based on star-QAM signaling constellations," IEEE Trans. Veh. Technol., vol. 67, no. 11, pp. 10543-10553, Nov. 2018.

[185] E. Sfeir, R. Mitra, G. Kaddoum, and V. Bhatia, "Performance analysis of maximum-correntropy based detection for SCMA," IEEE Commun. Lett., pp. 1-1, 2020.

[186] B. Ling, C. Dong, J. Dai, and J. Lin, "Multiple decision aided successive interference cancellation receiver for NOMA systems," IEEE Wireless Commun. Lett., vol. 6, no. 4, pp. 498-501, Aug. 2017.

[187] Q. Wu, S. Zhang, B. Zheng, C. You, and R. Zhang, "Intelligent reflecting surface-aided wireless communications: A tutorial," IEEE Trans. Commun., vol. 69, no. 5, pp. 3313-3351, May 2021.

[188] R. Liu, M. Li, Q. Liu, A. L. Swindlehurst, and Q. Wu, "Intelligent reflecting surface based passive information transmission: A symbollevel precoding approach," IEEE Trans. Veh. Technol., vol. 70, no. 7, pp. 6735-6749, Jul. 2021.

[189] Z. Wang, Y. Liu, X. Mu, Z. Ding, and O. A. Dobre, "NOMA empowered integrated sensing and communication," IEEE Commun. Lett., pp. 1-1, Jan. 2022. 


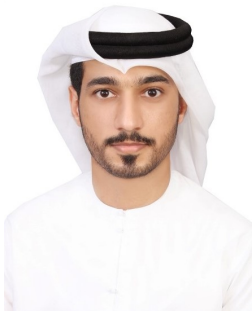

Hamad Yahya (Graduate Student Member, IEEE) was born in Sharjah, UAE, in 1997. He received the B.Sc. degree in electrical engineering/communication from Ajman University, Ajman, UAE, in 2018, where he was the top of the class and the M.Sc. degree (with Distinction) in communications and signal processing from The University of Manchester, Manchester, U.K., in 2019, where he is currently pursuing the $\mathrm{Ph} . \mathrm{D}$. degree in electrical and electronic engineering. He is also a Graduate Teaching Assistant with The University of Manchester. His research interests include wireless communications for future wireless networks, optimization, and visible light communication.

Mr. Yahya received the Best Student and the Best Project Prizes from the Department of Electrical and Electronic Engineering, The University of Manchester. He was nominated for the Distinguished Achievement Award in 2019, where he was among the top 14 postgraduate taught students across the Faculty of Science and Engineering. He is the recipient of the M.Sc. Success Scholarship from The University of Manchester.

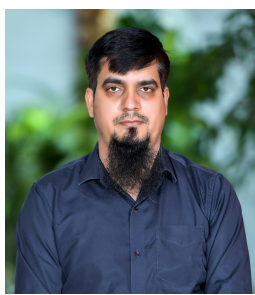

Ashfaq Ahmed (Senior Member, IEEE) earned the M.S. and Ph.D. Degrees from the Department of Electronics and Telecommunications, Politecnico di Torino, Torino, Italy, in 2010 and 2014, respectively. He is currently affiliated with the Khalifa University (KU) Department of Electrical Engineering and Computer Science's Center for Cyber-Physical Systems. Prior to joining KU, he was an Assistant Professor at the Department of Electrical \& Computer Engineering, COMSATS University Islamabad, Wah campus, Pakistan, for almost six years. His current research interests include applied optimization for telecommunications and smart system applications, physical layer wireless communications, ASIC and FPGA design and implementation of digital signal processing architectures, particularly those closely related to digital communications and video and image coding.

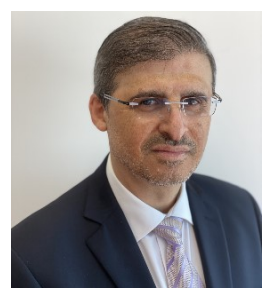

Arafat Al-Dweik (Senior Member, IEEE) received the M.S. (Summa Cum Laude) and Ph.D. (Magna Cum Laude) degrees in electrical engineering from Cleveland State University, Cleveland, OH, USA, in 1998 and 2001, respectively.

He is currently with the Department of Electrical Engineering and Computer Science, Khalifa University, Abu Dhabi, UAE. He also worked at Efficient Channel Coding, Inc., Cleveland, OH, USA, Department of Information Technology, Arab American University, Jenin, Palestine, and University of

Guelph, ON, Canada. He is a Visiting Research Fellow with the School of Electrical, Electronic, and Computer Engineering, Newcastle University, Newcastle upon Tyne, U.K, and a Research Professor with Western University, London, ON, Canada, and University of Guelph, Guelph, Canada. He has extensive research experience in various areas of wireless communications that include modulation techniques, channel modeling and characterization, synchronization and channel estimation techniques, OFDM technology, error detection and correction techniques, MIMO, and resource allocation for wireless networks.

Dr Al-Dweik serves as an Associate Editor for the IEEE Transactions on Vehicular Technology and the IET Communications. He is a member of Tau Beta Pi and Eta Kappa Nu. He was awarded the Fulbright scholarship from 1997 to 1999. He was the recipient of the Hijjawi Award for Applied Sciences in 2003, Fulbright Alumni Development Grant in 2003 and 2005, Dubai Award for Sustainable Transportation in 2016, UAE Leader-Founder Award in 2019. $\mathrm{He}$ is a Registered Professional Engineer in the Province of Ontario, Canada.

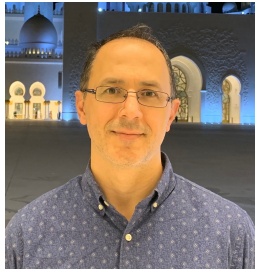

Emad Alsusa (Senior Member, IEEE) completed a $\mathrm{PhD}$ in Telecommunications from the University of Bath in the United Kingdom in 2000 and in the same year he was appointed to work on developing high data rates systems as part of an industrial project based at Edinburgh University. He joined Manchester University (then UMIST) in September 2003 as a faculty member where his current rank is a Reader in the Communication Engineering Group. His research interests lie in the area of Communication Systems with a focus on Physical, MAC and Network Layers including developing techniques and algorithms for array signal detection, channel estimation and equalization, adaptive signal precoding, interference avoidance through novel radio resource management techniques, cognitive radio and energy and spectrum optimization techniques. Applications of his research include cellular networks, IoT, Industry 4.0, and Powerline Communications. Emad's research work has resulted in over 200 journals and refereed conference publications mainly in top IEEE transactions and conferences. Emad has supervised over $30 \mathrm{PhDs}$ to successful completion. Emad is an Editor of the IEEE Wireless communication Letters, a Fellow of the UK Higher Academy of Education, and a TPC Track Chair of a number of conferences such as VTC'16, GISN'16, PIMRC'17 and Globecom'18, as well as the General Co-Chair of the OnlineGreenCom' 16 Conference. He is currently the UK representative in the International Union of Radio Science, and a Co-Chair of the IEEE Special Working Group on RF Energy Harvesting. Emad has received a number of awards including the best paper award in the international Symposium on Power Line Communications 2016 and the Wireless Communications and Networks Conference 2019. 